$$
\text { D. \#3097 }
$$

\title{
Fusion Reactivity Graphs and Tables for Charged Particle Reactions
}

J. Rand McNally, Jr. 


\section{DISCLAIMER}

This report was prepared as an account of work sponsored by an agency of the United States Government. Neither the United States Government nor any agency Thereof, nor any of their employees, makes any warranty, express or implied, or assumes any legal liability or responsibility for the accuracy, completeness, or usefulness of any information, apparatus, product, or process disclosed, or represents that its use would not infringe privately owned rights. Reference herein to any specific commercial product, process, or service by trade name, trademark, manufacturer, or otherwise does not necessarily constitute or imply its endorsement, recommendation, or favoring by the United States Government or any agency thereof. The views and opinions of authors expressed herein do not necessarily state or reflect those of the United States Government or any agency thereof. 


\section{DISCLAIMER}

Portions of this document may be illegible in electronic image products. Images are produced from the best available original document. 
Printed in the United States of America. Available from National Technical Information Service

U.S. Department of Commerce

5285 Port Royal Road, Springfield, Virginia 22161

Price: Printed Copy $\$ 5.25$; Microfiche $\$ 3.00$

$$
4.5-0
$$

This report was prepared as an account of work sponsored by an agency of the United States Government. Neither the United States Government nor any agency thereof, nor any of their employees, contractors, subcontractors, or their employees, makes any warranty, express or implied, nor assumes any legal liability or responsibility for any third party's use or the results of such use of any information, apparatus, product or process disclosed in this report, nor represents that its use by such third party would not-infringe privately owned rights. 
ORINL/TM-5914

Dist. Category UO-20 $3, d, B$

Contrast No. W-740j-enz-26

FUSION REACIIVITY GRAPHS AND TABLES FOR CHARGED PARTICLE REACTIONS

J. Rand MoNally, Jr.

Fusion Enargy Division

K. E. Rothe and R. D. Sarp

Computer Soiences Division

Date Published - August 1979

NOTICE This document contains information of a preliminary nature. $I t$ is suliject to revision or corrootion and therofore does not represent a final report.

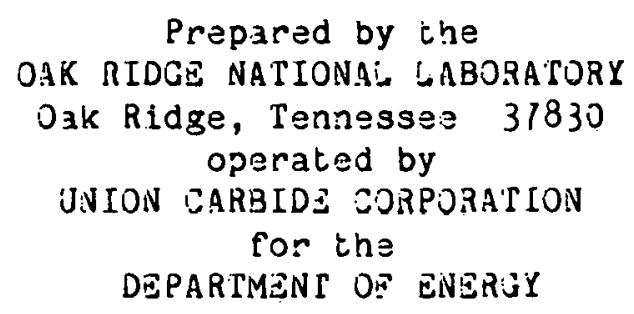


THIS PAGE

WAS INTENTIONALLY

LEFT BLANK 


\section{CONTENTS}

ABSTRACT $\ldots \ldots \ldots \ldots \ldots \ldots \ldots \ldots \ldots \ldots \ldots \ldots \ldots \ldots \ldots \ldots \ldots \ldots \ldots \ldots \ldots \ldots \ldots \ldots$

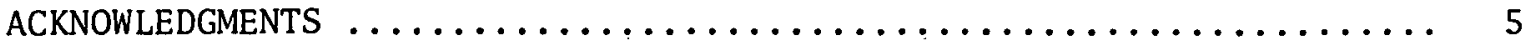

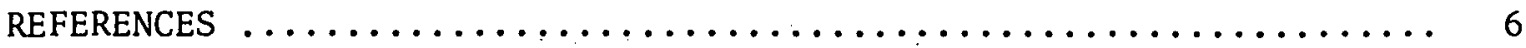

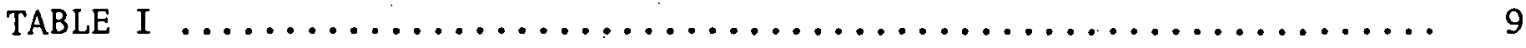

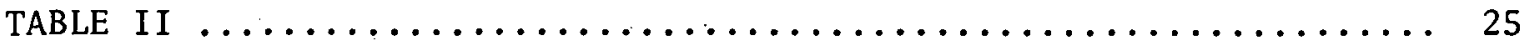

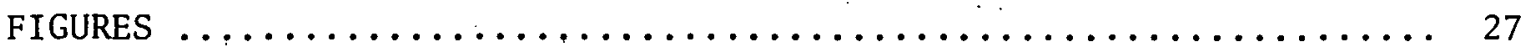




\section{THIS PAGE}

WAS INTENTIONALLY

\section{LEFT BLANK}




\begin{abstract}
Graphs and tables are presented on 31 light isotope fusion reaction parameters : $\langle\sigma v\rangle, n\langle\sigma v\rangle,\langle\sigma v\rangle Q_{+}, n\langle\sigma v\rangle Q_{+}$(for $n=10^{20}$ fuel ion species $/ \mathrm{m}^{3}$ and $Q_{+}=$energy release in eharged particles)] in the kinetic temperature range 1 to $1000 \mathrm{keV}$.
\end{abstract}


The O-D (point model) advaneed fusion fuels computer code surrently in use at the Oak Ridge National Laboratory incorporates 31 light isotope raactions. This code uses the reactivity parameter 〈 $\sigma v\rangle$ based on Maxwellian ion velocity distributions as a function of kinetic temperature from $T=1$ to $1000 \mathrm{keV}$. Tabular data are given for $\langle\sigma \mathrm{v}\rangle$, as are graphs of $\langle\sigma v\rangle, n\langle\sigma v\rangle,\langle\sigma v\rangle Q_{+}$, and $n\langle\sigma v\rangle Q_{+}$(where $n=10^{20}$ fuel Ion species $/ \mathrm{m}^{3}$ and $Q_{+}$is the energy release in charged particles).

Inasmuch as these tables may provide a useful mini-handbook of fusion reaction data, it was deemed advisable to provide appropriate graphs as well as the basic 〈 $\sigma v\rangle$ data tables in summary form to members of the fusion energy research community for comment, correction, and (most hopefully) upgrading of the basic $\langle\sigma v\rangle$ data.

It is hoped that the present survey will prompt nuslear data experts to expand and improve the scope of this technical memo so as to incorporate charged particle nuclear cross-section data of prime interest to both fusion researchers and nuclear astrophysicists. Since suprathermal or "fast" fusion reactions are becoming inoreasingly important and require cross sections or $\sigma v$ values for energies up to about $15 \mathrm{MeV}$ for the fast particle, a more comprehensive "barn book" analogous to BNL-325 ("Neutron Cross Sections") should eventually incorporate this additional information. Interest has also been expressed for the temperature range below 1 keV for pellet fusion research (but see Refs. 1 and 2) and above $T=1 \mathrm{MeV}$ for both fusion and astrophysical applications.

The earliest survey of light isotope reaction parameters for fusion applications may be the 1954 tables of Tuck, ' who listed 
thermonuclear reaction rate parameters, $\langle\sigma v\rangle$, for $D D, D T, D^{3} \mathrm{He}$, and $\mathrm{Tr}$ for temperatures from $0.05 \mathrm{keV}$ to $100 \mathrm{keV}$. In 1956 Thompson $^{2}$ gave

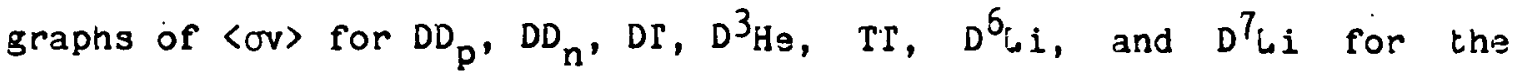
temperature range $0.01 \mathrm{keV}$ to $100 \mathrm{keV}$; this was subsequently revised and published in the open literature in 1957.3 Also, at about this time the extensive Jarnie and Seagrave plots of charged particle cross sections appeared as $6 A-2014 .{ }^{4}$ A summary of cross-section graphs and tables and graphs of Maxwell averaged $\langle\sigma v\rangle$ values based on LA-2014 was published by Greena; ${ }^{5}$ this report included $D D_{p}, D_{n}$, Dr, D ${ }^{3} \mathrm{He}, \mathrm{I}^{\prime} \mathrm{l}$, $T^{3} \mathrm{He}, D^{\sigma_{L}} i_{n}, D^{\sigma_{L}} i_{p}, D^{\sigma_{i} i_{t}}$, and $D^{\sigma_{i} i_{\alpha}}$ with $\langle\sigma v\rangle$ tabulated from 1 to 1000 keV.

Later published summaries insluded that of Crocker, Blow, and Watson; ${ }^{6}$ Duane; ${ }^{7}$ Miley, Towner, and Ivich; ${ }^{8}$ and Zimmerman, Fowler, and Caughlan ${ }^{9}$ - the latter being of special interest to astrophysicists and somewhat more extensive in heavy particle reaction data. No ext.ensive saarcin of the forelgn literature has been made and we apologize for any omissions. Also included in our tables are some new data (in Table I) and the graphs (see references at the end of rable II). Table II summarizes the reactions incorporated in Table I.

Table I incorporates 31 light. isotope rezotion ratc parameters, 〈ov〉 in $\mathrm{m}^{3} / \mathrm{sec}$, vs kinetic temperature, $\mathrm{T}$, from 1 to $10 \mathrm{keV}$ with interpolated values every $0.2 \mathrm{keV}$, from 10 to $100 \mathrm{keV}$ with interpolated values every $2 \mathrm{keV}$, and from 100 to $1000 \mathrm{keV}$ with interpolated values every $20 \mathrm{keV}$. Here, $T=2 / 3$ E. For interpolation the logs of the 〈ov〉 vs $T$ values were interpolated between entries in the left-hand 〈 $\sigma v\rangle$ column (2nd column) by the cubic spline method of H. Akima, ${ }^{10}$ as 
available in the International Mathematical Subroutine Library. Table I is also avallable on the National Magnetic Fusion Energy Computer Center (NMEECC) A7600 computer from filem (user \#26660, directory JRM1) and is named NSIGVDAT. As new (or useful older) data becomes available this filem wll be updated and possibly expanded. Updated outputs can be made available on request for those not having aceess to the NMEECC A76́00 computer.

Figures $1(a)-1(f)$ depict $\langle\sigma v\rangle$ and $n\langle\sigma v\rangle\left(n=10^{20} \mathrm{~m}^{-3}\right)$ for the various reactions in selected combinations of related reactions. Figures $2(a)-2(f)$ give $\langle\sigma v\rangle Q_{+}$and $n\langle\sigma v\rangle Q_{+}$, where $Q_{+}$(in $M e V$ ) is the chacged particle energy release only. The latter illustrates the relative dagree of reactivity in $a$ hot plasma induced by the charged reaction products.

It should be noted, nowever, that reactions involving pairs of like nuclei ocsur at least twice as often as reastions between pairs of unlike particles; i.e., the reaction rate is

$R . R .=\left\{\begin{array}{c}\frac{1}{2} n^{2}\langle\sigma v\rangle \quad \text { for like particles (e.g., DD) } \\ n_{1} n_{2}\langle\sigma v\rangle \\ \leq \frac{1}{4} n^{2}\langle\sigma v\rangle\end{array}\right\}$ for unlike particles (e.g., Dr),

where $n=$ fuel density $\left(=n_{1}+n_{2}\right)$. Thus, the $n\left\langle\sigma v>Q_{+}\right.$curves for DD $\left({ }^{3} \mathrm{He}{ }^{3} \mathrm{He}\right.$, etc.) and DD-CAT (CAT = catalyzed, meaning the first: generation products $\mathrm{T}$ and $3_{\mathrm{He}}$ are burned up as fast as they are 
generated ${ }^{11}$ ) underestimate the power deposition rate by at least a factor of two compared to DI for constant $n$.

Actual burn evaluations of $\mathrm{T}$ and ${ }^{3} \mathrm{He}$ burning indicate that about 50-80z of $\mathrm{T}$ and $25-50 \%$ of $3_{\mathrm{He}}$ may be consumed in fusion reactions in $z$ high temperature, steady-state DD burning mode. ${ }^{12}$ To satisfy the catalyzed burn condition the unburned $T$ and ${ }^{3}$ He need be isotopically separated from $p$ and $\alpha$ ashes and then fed as fuel make-up with new $D$ fuel to provide $a$ driven satalyzed burning condition (in a DI reastor unburned $\overline{\mathfrak{T}}$ must also be isotopically separated from the protons and also fed back with $D$. fuel). The fractional burn of tritium is approximately $F=n_{D} \tau_{p}\langle\sigma v\rangle /\left(1+n_{0} \tau_{p}\langle\sigma v\rangle\right)$ where $\tau_{p}=$ particle confinement time.

Tne ${ }^{3} \mathrm{He}+{ }^{6} \mathrm{Li}_{\mathrm{i}}$ reastion is incompletely known and data is only given for the $p+8_{B e^{0,1}}$ channels. Hopefully, because of its importance to detailed evaluation of the $p^{6} i \mathrm{i}$ chain reaction prospects, 13 new and extensive measurements will be made of che $d+$ $7 \mathrm{Be}$, the $p+2 \alpha$ three body breakup, and the $\alpha+5_{L i}$ channels, tne latter of which sould have the largest cross section according to Bromley and Almquist. ${ }^{14}$ sould and Boyce ${ }^{15}$ indicate that the three body breakup channel is roughly as probable as the $\mathrm{p}+{ }^{8} \mathrm{Be} e^{0,1}$ channels. The study by Gould and Boyce may not have included the very low energy proton spactrum from the $\alpha+{ }^{5} h_{i}\left({ }^{5} h_{i} \rightarrow p+\alpha\right)$ channel. The total $\langle\sigma v\rangle$ for ${ }^{3} \mathrm{He}+{ }^{6} \mathrm{Li}$ probably exceeds our tabulated values by a factor of at least two and possibly four or more (see Ref. 14, p. 273). The d+ 7 Be branch $(Q=+0.1 \mathrm{MeV})$ has a significant cross section up to at 
least $1.3 \mathrm{MeV} .{ }^{16}$ The nuclear physics of the complex $3_{\text {He }}+6_{\mathrm{Li}}$ reaction merits a comprehensive study.

Advanced pellet fuels may use $i \dot{i}, \mathrm{Be}, \mathrm{B}, \mathrm{C}$ and/or $\mathrm{N}$ as sarriers in various combinations of $D$ and $T$ as hydrides or hydrates [e.g., LiH, $\mathrm{BeH}_{2}, \mathrm{BH}_{3},\left(\mathrm{CH}_{2}\right)_{2}, \mathrm{CH}_{4}, \mathrm{NH}_{3}, \mathrm{LiBeH}_{3}, \mathrm{LiBH}_{4}$, etc.]. ${ }^{17}$ Side reactions such as $D^{6_{i}}, D^{7}{ }_{i}, p^{11_{B}}, p^{9} B e, t+7_{i} i$, etc. could be important contributors in the course of any large scale thermal excursions which may occur in some of tnese advaneed pellet fuels after ignition. For neutron induced reactions which can occur in very thick pellets (large $\dot{\rho}$ ) consult $\mathrm{BN} \mathrm{L}_{-}-325 .{ }^{18}$ such mixed pellet fuels have a higher ignition temperature than DT $\left(p_{0}=0.21 \mathrm{~g} / \mathrm{cm}^{3}, T_{\text {ign }}=4 \mathrm{keV}\right)$ because of the insreased bremsstrahlung radiation but offer much inoreased initial density ( $\rho_{0}$ up to $1.16 \mathrm{~g} / \mathrm{cm}^{3}, T_{\text {ign }} \simeq 10 \mathrm{keV}$ ); thus they permit redused pellet compression, ${ }^{19}$ i.e., $C=\left(\rho R_{i g n} / \rho_{o} R_{0}\right)^{3 / 2}$.

\section{ACKNOWLEDGMENTS}

We are indebted to C. R. Gould (North Carolina State University), A. J. Elwyn (Argonne National Laboratory), T. A. Tombrallo (California Institute of Technology), L. Ruby (University of California, Berkeley), and E. Norbeck (University of Iowa) for very useful nuclear dat.a inputs. He are also indebted to E. Teller (Lawrence inivermore Laboratory) and T. Samao (TRW, Redondo Beach, California) for helpful suggestions. 


\section{REFERENCES}

1. James i. Tuck, "Thermonuclear Reaction Rates," iAMS-1640, Los Alamos, New Mexico (March 1954)!

2. W. B. Thompson, "Thermonuclear Reaction Rates," AERE-T/M-138, Harwell, Berks, England (May 1956).

3. W: B. Tnompson, "Thermonuclear Reaction Rates," Proc. Phys. Soc. $703,1(1957)$.

4. Nelson Jarmie and John D. Seagrave, eds., "Wharged Partiole Cross Sections," iA-2014, Los Alamos, New Mexico (March 1956).

5. Samuəl L. Greene, Jr., "Maxwell Averaged Cross Sections for Some Thermonuclear Reactions on light Isotopes," UCRL-70522, Livermore, California (May 1957).

6. V. S. Crocker, S. Blow, and C. J. H. Watson, "Nuclear Cross-Section Requirements for Fusion Reactors," CLM-P240, Culnam Laboratory, Abingdon, Berkshire, England (1970).

7. B. H. Duane, "Fusion Cross Section Thoory," in BNill-1695, R1chland, Washington (November 1972).

8. George H. Miley, Harry Towner, and Nenad Ivich, "Fusion Cross Sections and Reactivities," C00-2218-17, Urbana, Illinois (June 1974). 9. Barbara A. Zimmerman, William A. Fowlen, and Goorgoanno R. Caughlan, "Tables of Inermonuclear Reaction Rates," OAP-399, Pasadena, California (March 1975). 〈uv> is given in $\mathrm{cm}^{3} \mathrm{~mole}^{-1} \mathrm{sec}^{-1}$ and $\mathrm{T}_{9}=$ $10^{-9} \mathrm{~T}_{\mathrm{K}}=11600 \times 10^{-5} \mathrm{~T}_{\mathrm{keV}}$.

10. H. Akima, "A New Method of Interpolation and Smooth Curve Fitting Based on iosal Procedures," J. ACM 17, 589 (1970). 
11. Robert G. Mills, "Catalyzed Deuterium Fusion Reastors," PPL-TM-259, Princeton, Naw Jersey (April 1971 ).

12. J. Rand MoNally, Jr., "Advaneed Fusion Fuels," Proc. 6th IEEE Symposium on Engineering Problems of Fusion Research, IEEE Pub. No. 75 CH 1097-5-NPS, p. 1012 (1970́).

13. J. Rand MaNally, Jr., "Some Thoughts on the $p\left({ }^{6} L_{i}, \alpha\right)^{3} H e\left({ }^{6} L_{i}\right.$, p) ${ }^{8} \mathrm{Be}$ Chain Reaction," paper presented at the 1979 IEEE International Conference on Plasma Science, Montreal, Canada, June 4-6, 1979.

14. D. A. Bromley and $\vec{E}$. Almquist; "He${ }^{3}$ Induced Reactions," Cnalk River Report C.R.P. 881 (AECL No. 950), Cnalk River, Ontario, Canada (1959).

15. C. R. Gould and J. R. Boyce, "Cross-Section Measurements for Cnarged Particle Fusion Reactors: The $6_{\mathrm{L} i}\left(\dot{3}_{\mathrm{H}}, \mathrm{p}\right) 2 \alpha$ Reastion, Nucl. Sci. Eng. 60ㅇ, 477 (1976).

16. M. R. Aleksic, R. V. Popie, D. M. Stenojevic, B. Z. Stepančić, "6 $6_{\mathrm{t}}\left({ }^{3} \mathrm{He}, d\right)^{7} \mathrm{Be}$ Reaction at Low Energies," Fizika 2, 113 (1970́).

17. J. Rand MeNally, Jr., and R. D. Sharp, "Advanced Fuels for Inertial Confinement," Nucl. Fusion 16, 868 (1976).

18. John R. Stehn, Murrey D. Goldberg, Benjamin A. Magurno, and Renate Wiener-Chasman, "Neutron Cross Sections," BNl-325, Brooknaven, Long Island, New York (May 1954).

19. Sea J. Rand MCNally, Jr., "Jeneral Requirements and Approaches to Advanced Fuel Fusion," in Proc. Review Meeting on Advanced-Fuel Fusion, EPRI ER-536-SR, p. 197, Electric Power Research Institute, Palo Alto, California, June 27-28, 1977. 
8

FIGURE CAPTIONS

Figs. $1(a)-1(f)$. Plots of Maxwellian averaged $\langle\sigma v\rangle$ and $n\langle\sigma v\rangle$

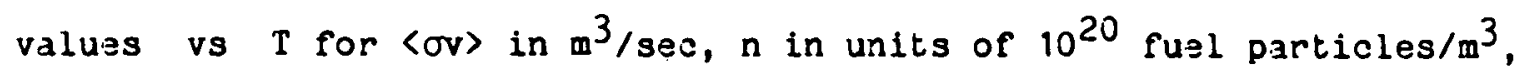
and $\mathrm{I}$ in $\mathrm{keV}$.

Figs. 2(a)-2(f).: Plots of $\langle\sigma v\rangle Q_{+}$and $n\langle\sigma v\rangle Q_{+}$values vs $T$ for my> in $\mathrm{m}^{3} / \mathrm{sec}, \mathrm{n}$ in units of $10^{20}$ fuel partioloo/m $\mathrm{m}_{+}$(charged particle energy release) in $\mathrm{MeV}$, and $\mathrm{T}$ in $\mathrm{keV}$. 
Table I. Reactions, <sigma v> tabular references, and selected energy releases in $\mathrm{keV}$ (inversely proportional to mass)

\begin{abstract}
136 = JMAX (\# ELEMENTS IN TABLE/REACTION): SEE DESCRIPTION AT EHD
$T$ (J) IN KEV SIGVI(J) IN MB/SEC 3-26-79

1.000000E+00 9.650900E-29 2.510000E-28 $5.750000 \mathrm{E}-28 \quad 1.100000 \mathrm{E}-27 \quad 1.900000 \mathrm{E}-2.7$

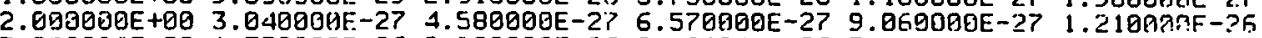

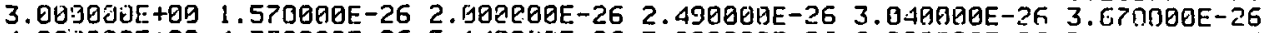

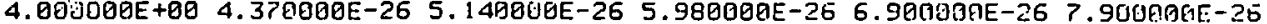

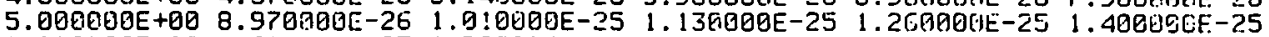

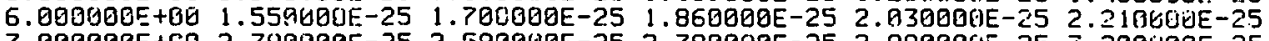

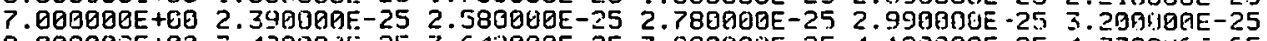

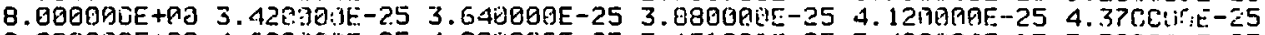

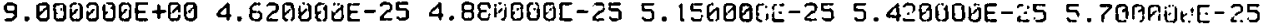

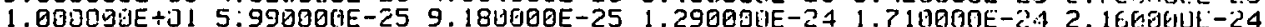

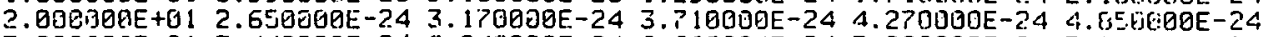

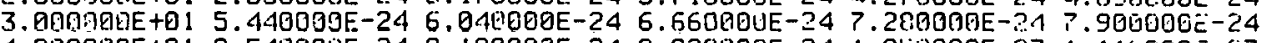

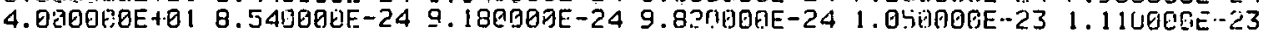

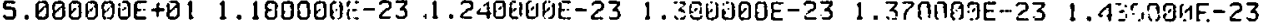

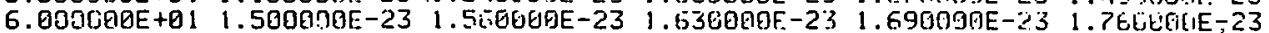

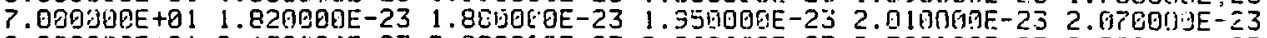

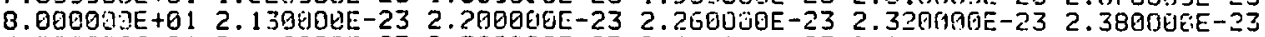

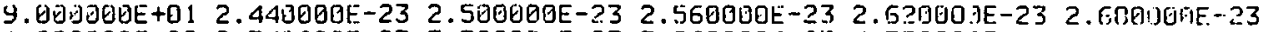

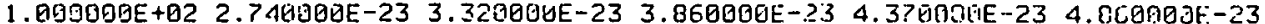

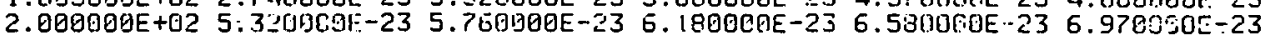

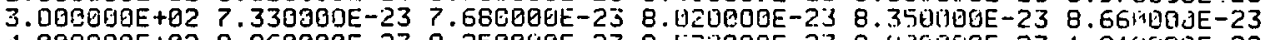

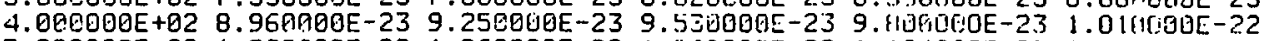

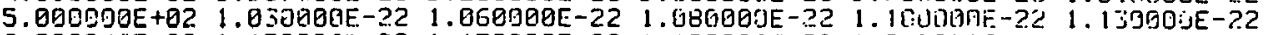

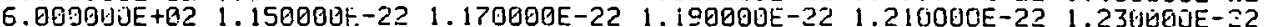

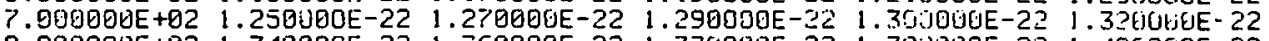

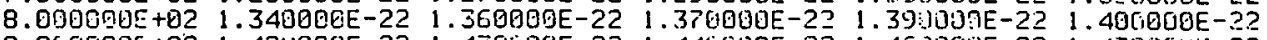

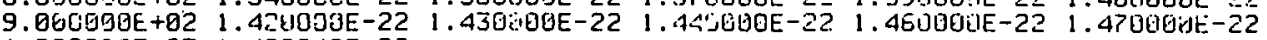
$1.090060 E+03 \quad 1.4809130 E-22$

$A 01$ SIGVI $D+D \Rightarrow 3 H E(817)+N(2450)$

REF 1
\end{abstract}

$T(J)$ IN KEV SIGV2(J) IN M3/SEC

$1.000000 \mathrm{E}+00$ 9.660000E-29 $2.610000 \mathrm{E}-28 \quad 5.760000 \mathrm{E}-28 \quad 1.100000 \mathrm{E}-27 \quad 1.910000 \mathrm{E}-27$ 2.000000E+ +00 3.850อ90E-27 4.590000E-27 6.580000E-27.9.070000E-27 1.210000E- 26

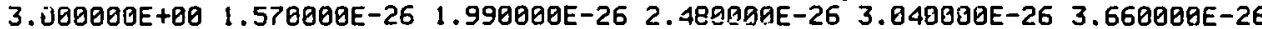
4. $000000 \mathrm{E}+00 \quad 4.350000 \mathrm{E}-26 \quad 5.110000 \mathrm{E}-26 \quad 5.950000 \mathrm{E}-26 \quad 6.860000 \mathrm{E}-26 \quad 7.840000 \mathrm{E}-26$ 5.

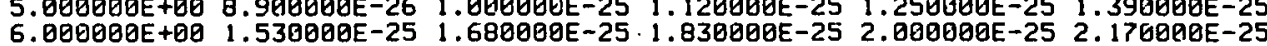
7. 8. Q00000E+00 3.330000E-25 $3.550000 \mathrm{E}-25 \quad 3.770000 \mathrm{E}-25 \quad 4$. .000000F-25 $4.230000 \mathrm{E}-25$

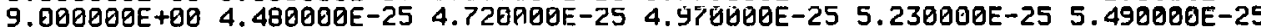

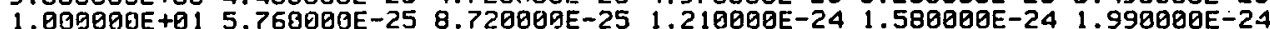

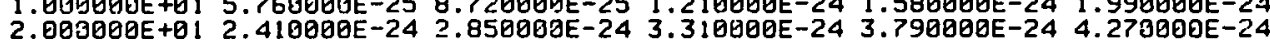
$\begin{array}{llllll}2.000000 E+0 & 2.410000 E-24 & 2.850000 E-24 & 3.310000 E-24 & 3.790000 E-24 & 4.273000 E-24 \\ 3.000000 E+01 & 4.760000 E-24 & 5.250000 E-24 & 5.750000 E-24 & 6.260000 E-24 & 6.770000 E-24\end{array}$ 4. घ000E0E+01 7.280000E-24 7.7900V0E-24 8.300000E-24 8.810000E-24 9.320000E-24 $5.000000 \mathrm{E}+01$ 9.940000E-24 $1.030000 \mathrm{E}-23 \quad 1.090000 \mathrm{E}-23 \quad 1.1140000 \mathrm{E}-23 \quad 1.190000 \mathrm{E}-23$

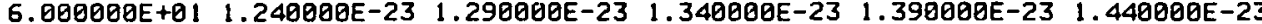

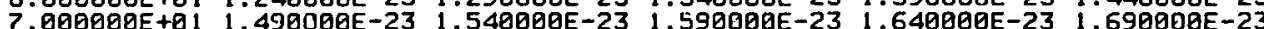

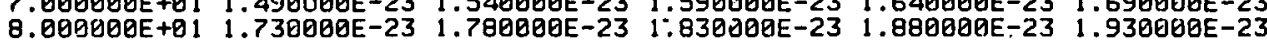

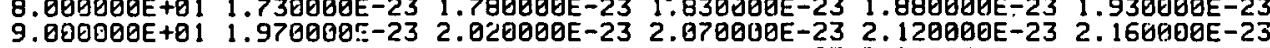
1.000000E+02 2.21000 AE $-23 \quad 2.660000 \mathrm{E}-23 \quad 3.099000 \mathrm{E}-23 \quad 3.510000 \mathrm{E}-23 \quad 3.900000 \mathrm{E}-23$ $2.000000 \mathrm{E}+02$ 4.290000E-23 $4.650006 \mathrm{E}-23$ 5.010000E-23 $5.350000 \mathrm{E}-23 \quad 5.680000 \mathrm{E}-23$ 3. $900000 \mathrm{E}+02$ 6.

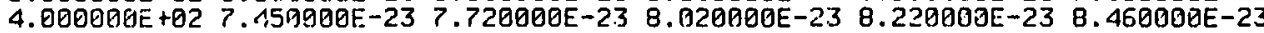

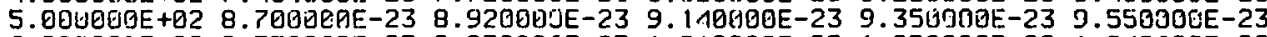

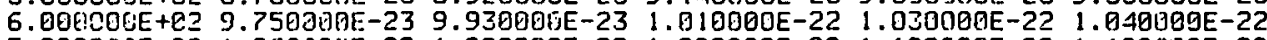

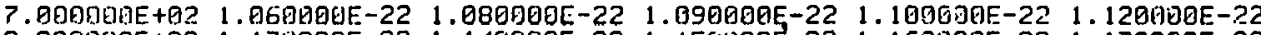
8.000000E+02 1.130000E-22 1.140000E-22 1.150000E-22 1.160000E-22 1.170090E-22 $9.000000 \mathrm{E}+02$ 1.19日009E-22 1.130000E-22 1.200000E-22 1.200000E-22 1.210000E-22 $1 . \boxminus 6 \boxminus \boxminus \boxminus \boxminus E+03 \quad 1.220000 \mathrm{E}-22$ 
Table I (continued)

T(J) IN KEV SICV3(J) IN M3/SEC

1.000000E+00 6.270000E-27 $1.860009 E-26 \quad 4.440000 \mathrm{E}-26 \quad 9.110000 \mathrm{E}-26 \quad 1.670000 \mathrm{E}-25$

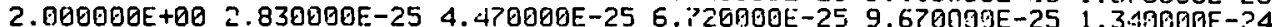
$3.000090 \mathrm{E}+00$ 1.810000E-24 $2.380000 \mathrm{E}-24$ 3.060000E-24 3.800.700E-24 4.790900E-34 4.000000E+00 5.860000E-24 7.070900E-24 8.430000E-24 9.959000E-24 1.1EveEE0E-23

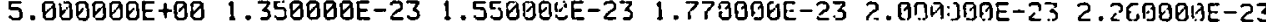

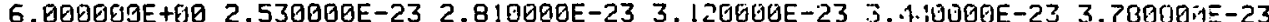

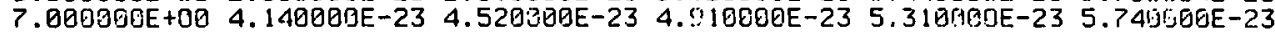

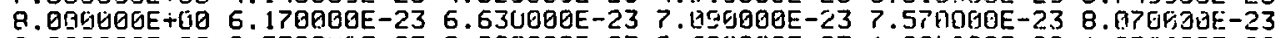

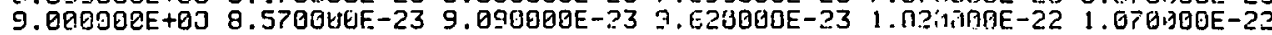

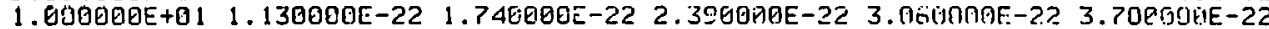

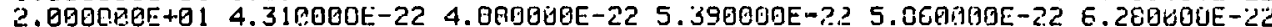

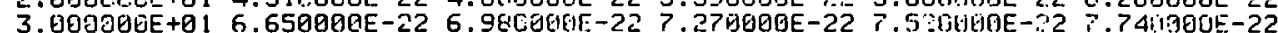

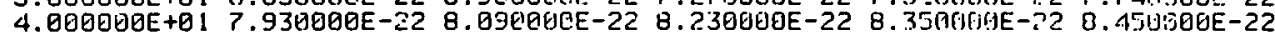

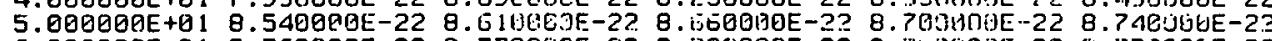

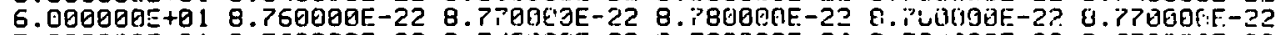

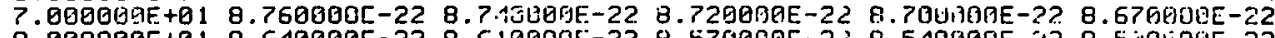

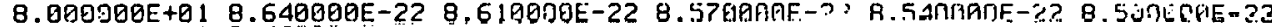

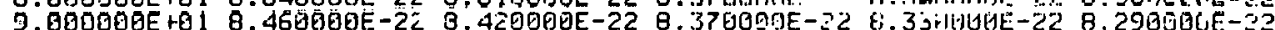

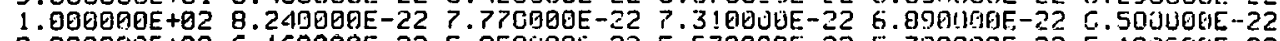

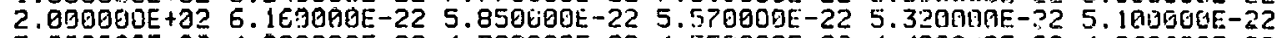

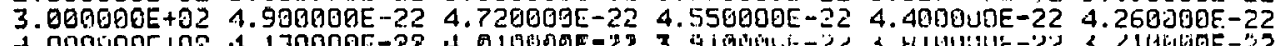

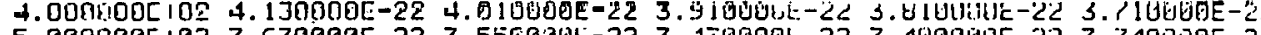

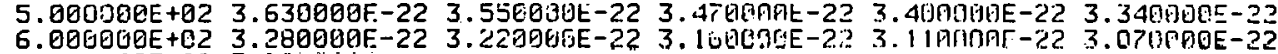

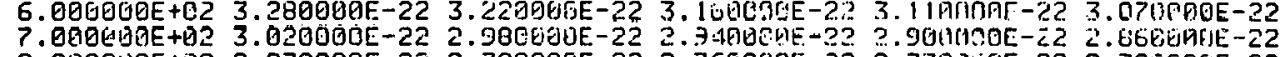

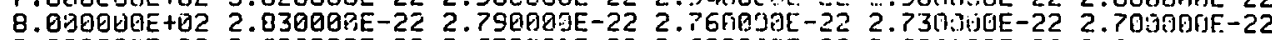
9.

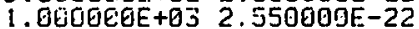

$A 03$ SIGV3 $D+T \Rightarrow A(3517)+N(14969)$

PEF 1

$T(J)$ IN KEV SIGV4(J) IN M3/SEC

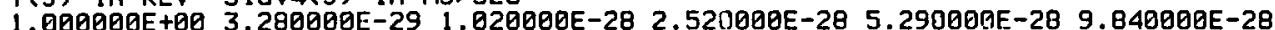

2.000000E+00 1.600000E-27 2.660000E-27 4.000000E-27 $5.750009 E-27 \quad 7.960000 \mathrm{E}-27$

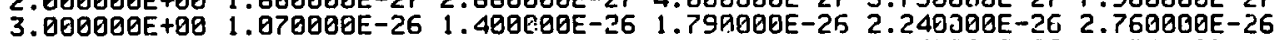
4.000000E+00 3.350000E-26 4.010000E-26 4.750000E-26 5.560000E-26 $6.450000 \mathrm{E}-26$ 5.000000E+00 $7.420000 \mathrm{E}-26 \quad 8.470000 \mathrm{E}-26 \quad 9.600000 \mathrm{E}-26 \quad 1.080900 \mathrm{E}-25 \quad 1.210000 \mathrm{E}-25$

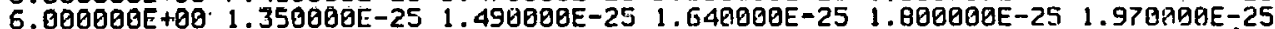
7. 000000E+ $2.140000 \mathrm{E}-25 \quad 2.32000 \mathrm{BE}-25 \quad 2.510000 \mathrm{E}-25 \quad 2.710009 \mathrm{E}-252.910000 \mathrm{E}-25$ B. .00000E+00 3.120000E-25 3.340000E-25 3.560000E-25 $3.790000 \mathrm{E}-25$ 4.030000E-25 $9.000000 \mathrm{E}+00$ 4.270000E-25 4.520000E-25 4.770000E-25 5.03901UEE-25 5.3MA000E-25 1. $000000 \mathrm{E}+01$ 5.570000E-25 $\quad$ B.540000E-25 $1.190000 \mathrm{E}-24 \quad 1.560000 \mathrm{E}-24 \quad 1.960000 \mathrm{E}-24$

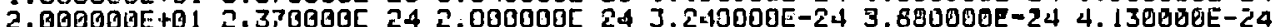
3.

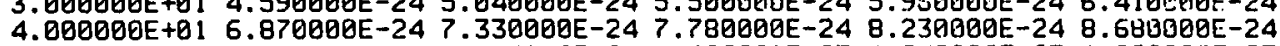
5.000900E+01 $9.120000 \mathrm{E}-24$ 9.570000E-24 $1.00000 \mathrm{EE}-23 \quad 1.040900 \mathrm{E}-23 \quad 1.090000 \mathrm{E}-23$

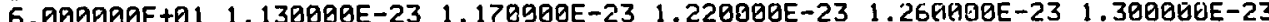

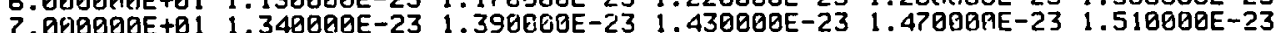
$\begin{array}{llllll}7.000000 E+01 & 1.340000 E-23 & 1.390000 E-23 & 1.430000 E-23 & 1.670000 E-23 & 1.710000 E-23\end{array}$ 0.900000E+02 $0.175000 \mathrm{E}-22$ 0.179200E-22 0.183400E-22 0.187400E-22 0.191200E-22 $0.100000 \mathrm{E}+03$ 0.195000E-22 $0.234800 \mathrm{E}-22$ 0.279400E-22-0.327800E-22 $0.377800 \mathrm{E}-22$

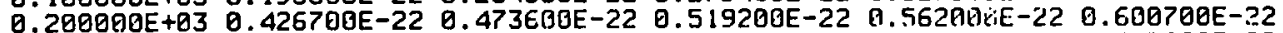

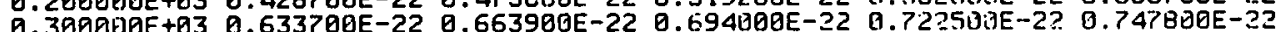

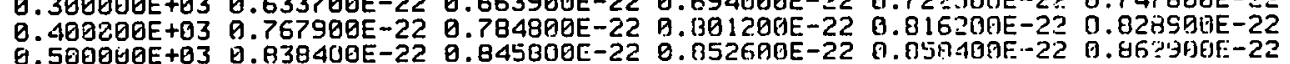
$0.6905100 \mathrm{E}+03$ 9.865500E-22 $9.867100 \mathrm{E}-22$ 0. $668400 \mathrm{E}-22$ !.

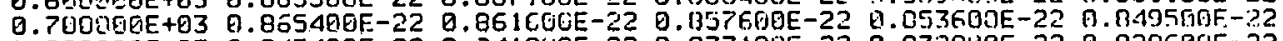

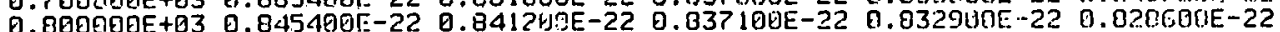
$0.900000 \mathrm{E}+23$ Q.824200E-22 0.819300E-22 月.813700E-22 0.807600E-22 0.801000E-22 $0.900000 E+23$
$0.100000 \mathrm{~B}+04$
$0.794300 \mathrm{E}-22$

A04 SIGV4 $T+T \Rightarrow A(1259)+N(5034)+N(5034)$

REF 1.2 
Table I (continued)

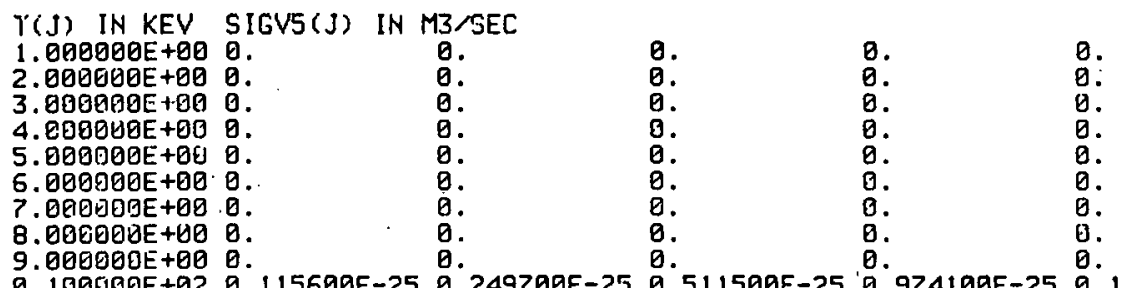

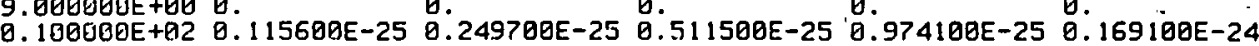
$0.200000 \mathrm{E}+02$ 0.262300E-24 $0.376600 \mathrm{E}-24 \quad 0.523900 \mathrm{E}-24 \quad 0.704200 \mathrm{E}-24 \quad 0.911800 \mathrm{E}-24$ $0.300000 \mathrm{E}+02 \quad 0.113400 \mathrm{E}-23 \quad 0.138200 \mathrm{E}-23 \quad 0.168200 \mathrm{E}-23 \quad 0.202000 \mathrm{E}-23 \quad 0.241100 \mathrm{E}-23$ $0.490900 \mathrm{E}+02 \quad 0.280500 \mathrm{E}-23 \quad 0.322000 \mathrm{E}-23 \quad 0.368600 \mathrm{E}-23 \quad 0.415509 \mathrm{E}-23 \quad 0.473502 \mathrm{E}-23$ $0.590000 \mathrm{E}+02$ 0.528700E-23 $0.586100 \mathrm{E}-23 \quad 0.648000 \mathrm{E}-23 \quad 0.7137 \mathrm{GQE}-23 \quad 0.782100 \mathrm{E}-23$ $0.600900 \mathrm{E}+02 \quad 0.851500 \mathrm{E}-23 \quad 0.923000 \mathrm{E}-23 \quad 0.398500 \mathrm{E}-23 \quad 0.107700 \mathrm{E}-22 \quad 0.115800 \mathrm{E}-22$

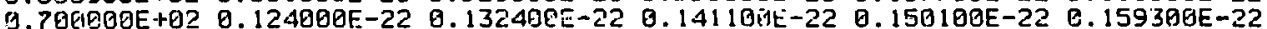
$0.800000 \mathrm{E}+02$ 0.168500E-22 0.17780EE-22 0.187400E-22 0.197200E-22 0.2074U0E-22 $0.900000 \mathrm{E}+02$ 0.217B00E-22 0.228500E-22 $0.239400 \mathrm{E}-22$ 0.250300E-22 $0.260900 \mathrm{E}-22$

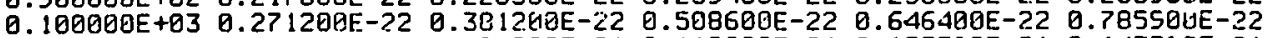

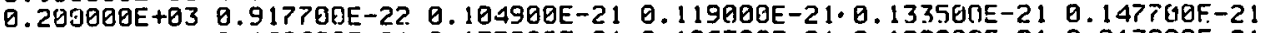

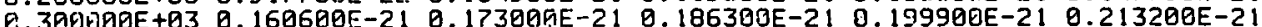
. 3 A

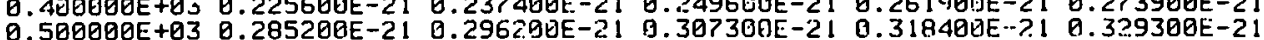

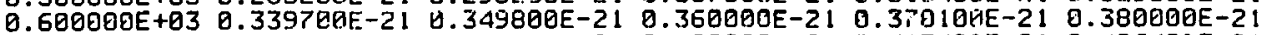
$0.700000 \mathrm{E}+03$ 0.389500E-21 $0.398000 \mathrm{E}-21 \quad 0.408200 \mathrm{E}-21 \quad 0.41749 \mathrm{AEE}-21 \quad 0.426400 \mathrm{E}-21$ $\begin{array}{lllllll}0.800000 \mathrm{E}+03 & 0.435200 \mathrm{E}-21 & 0.443800 \mathrm{E}-21 & 0.4523 \mathrm{HOE}-21 & 0.460000 \mathrm{E}-21 & 0.469100 \mathrm{E}-21\end{array}$ $0.900000 E+03 \quad 0.477200 E-21 \quad 0.485200 E-21 \quad 0.493100 E-21 \quad 0.500900 E-21 \quad 0.508600 E-21$

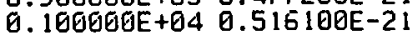

AOS SIGV5 T+3HE $\Rightarrow D(9546)+A(4773)$

$P(5374)+A(1344)+N(5374)$

$P(10077)+A(403)+N(1612)$
$41 \%$ OF SIGVS $55 \%$ OF SIGV5 4\% OF SIGVS
REF 2

REF 2

$T(\mathrm{~J})$ IN KEV SIGVG (J) IN M3/SEC

1 J IN KEV 3 . 2.000000E+QB $1.410000 \mathrm{E}-292.940000 \mathrm{E}-295.030000 \mathrm{E}-29$ 1.010000E-20 $1.700000 \mathrm{E}-28$

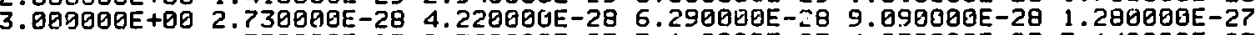
4.020000E+50 1.750000E-27 2.360000E-27 3.110000E-27 4.030000E-27 5.1.19000E-27 5. $000000 \mathrm{E}+00$ 6.460000E-27 $8.030000 \mathrm{E}-27 \quad 9.870000 \mathrm{E}-27 \quad 1.200000 \bar{c}-25 \quad 1.45000 \mathrm{at}-26$

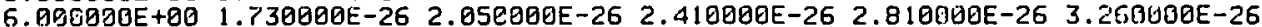

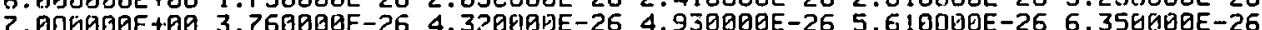
7.

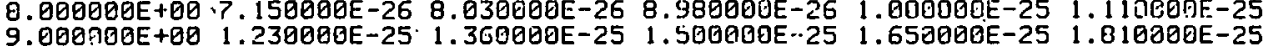

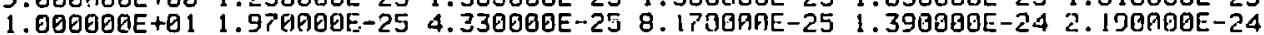
$2.000000 \mathrm{t}+013.260000 \mathrm{E}-24 \quad 4.610000 \mathrm{E}-24 \quad 6.280000 \mathrm{E}-24 \quad 8.270000 \mathrm{E}-24 \quad 1.060000 \mathrm{E}-23$

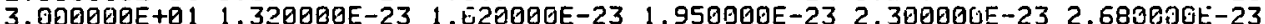

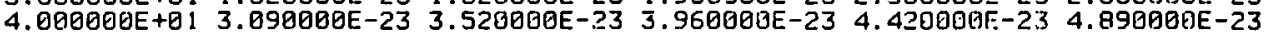

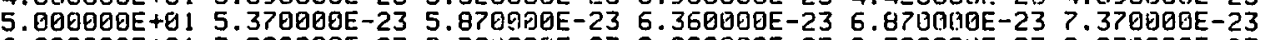
6.000000E+01 7.88000QE-23 8.33UD0EE-23 8.88000QE-23 $9.38000 \mathrm{DIE}-23$ 9.870000E-23

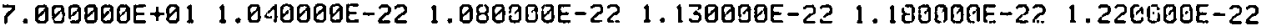

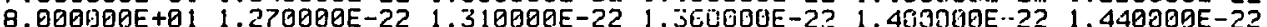
$9.000000 \mathrm{E}+01$ 1.480000E-22 $1.520000 \mathrm{E}-22 \quad 1.560000 \mathrm{E}-2 ?$ ? $1.600000 \mathrm{E}-22 \quad 1.636503 \mathrm{E}-22$

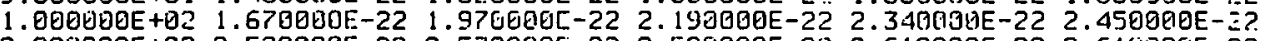

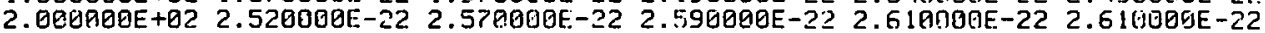

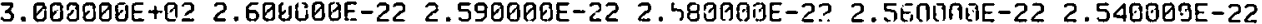

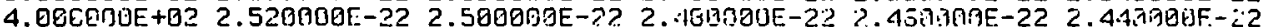

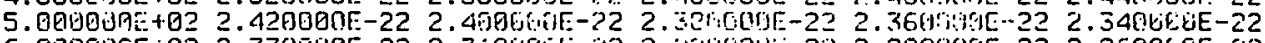

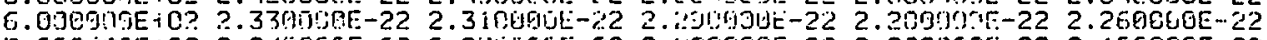

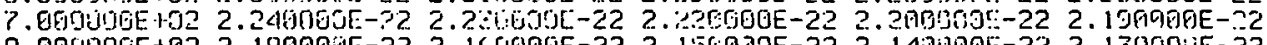

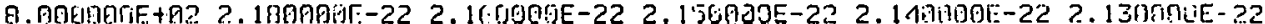

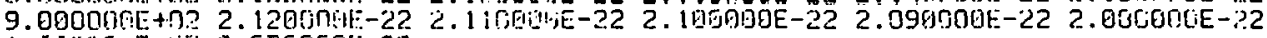

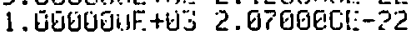


Table I (continued)

$T(J)$ IN KEV SIGVP(J) IN $M 3 / S E C$

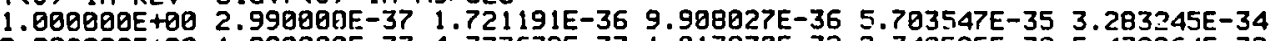

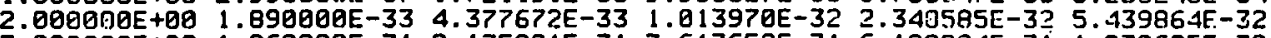
3.000000E+00 $1.260000 \mathrm{E}-312.13500 \mathrm{jE}-31 \quad 3.617652 \mathrm{E}-31 \quad 6.129924 \mathrm{E}-31$ 1. $230685 \mathrm{E}-30$ 4. $990000 \mathrm{E}+00 \quad 1.760000 \mathrm{E}-302.548315 \mathrm{E}-30 \quad 3.689727 \mathrm{E}-30 \quad 5.342387 \mathrm{E}-30 \quad 7.735287 \mathrm{E}-30$ $5.006000 \mathrm{E}+00$ 1.120000E-29 $1.483028 \mathrm{E}-29$ 1.963886E-29 $2.690553 \mathrm{E}-29 \quad 3.443629 \mathrm{E}-29$

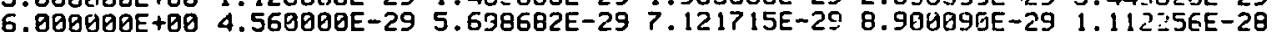
$7.000906 \mathrm{E}+00$ 1.390000E-28 $1.668118 \mathrm{BE}-28$ 2.001885E-28 $2.402432 \mathrm{E}-28 \mathrm{C} 2.883124 \mathrm{E}-28$

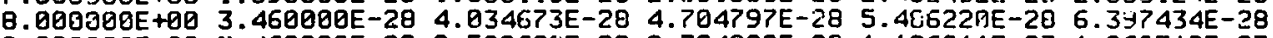
$9.000000 \mathrm{E}+00 \mathrm{7} .460006 \mathrm{E}-28$ 8.508699E-28 $9.704829 \mathrm{E}-28 \quad 1.106911 \mathrm{E}-27 \quad 1.262518 \mathrm{E}-27$

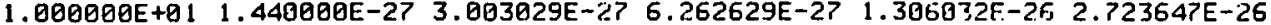
$0.200000 \mathrm{E}+02 \quad 0.568000 \mathrm{E}-25 \quad 0.842800 \mathrm{E}-25 \quad 0.122200 \mathrm{E}-24 \quad 0.1721 \mathrm{AOE}-2.4 \quad 0.234100 \mathrm{E}-24$ $0.300000 \mathrm{E}+02$ 0.306000E-24 0.388200E-24 0.484600E-24 $0.594600 \mathrm{E}-24$ 0.7 $1400 \mathrm{FE}-24$

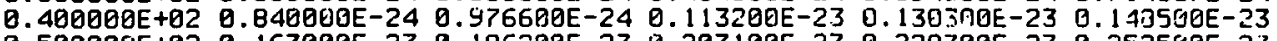

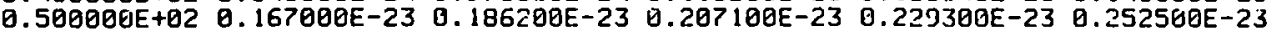
$\begin{array}{llllll}0.600000 \mathrm{t}+02 & 0.276900 \mathrm{E}-23 & 0.300300 \mathrm{E}-23 & 0.326000 \mathrm{E}-23 & 0.352900 \mathrm{E}-23 & 0.300400 \mathrm{E}-23\end{array}$ $0.700000 \mathrm{E}+02 \quad 0.408000 \mathrm{E}-23 \quad 0.436000 \mathrm{E}-23 \quad 0.465100 \mathrm{E}-23 \quad 0.495000 \mathrm{E}-23$ 0.525403E-23 $0.800000 \mathrm{E}+02 \quad 0.556000 \mathrm{E}-23 \quad 0.586800 \mathrm{E}-23 \quad 0.618400 \mathrm{E}-23 \quad 0.650 \mathrm{PBOE}-23 \quad 0.603900 \mathrm{E}-23$ $0.9000 \mathrm{JOE}+02$ 0.718000E-23 $0.75290 \mathrm{EE}-23$ 0.788000E-23 $0.02290 \mathrm{AE}-23$ 0.856E00E-23

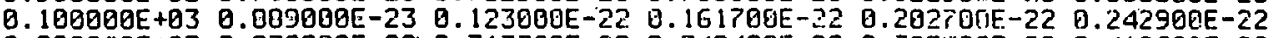

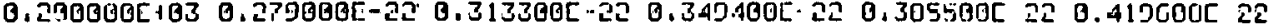
$0.300000 \mathrm{E}+03 \quad 0.449000 \mathrm{E}-22 \quad 0.476200 \mathrm{E}-22 \quad 0.564900 \mathrm{~F}-22 \quad 0.533700 \mathrm{E}-22 \quad 0.561 \mathrm{AMAE}-223$ $0.480000 \mathrm{E}+03 \quad 0.505000 \mathrm{E}-22 \quad 0.606900 \mathrm{E}-22 \quad 0.629200 \mathrm{E}-22 \quad 0.651200 \mathrm{E}-22 \quad 0.672400 \mathrm{E}-22$ $0.500000 \mathrm{E}+03$ 0.692000E-22 0.7 10800E-22 $0.729700 \mathrm{E}-22$ 0.740120E-22 0.76630GE-22

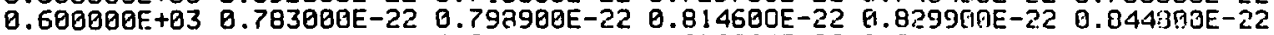
$0.700000 \mathrm{E}+03$ 0.859000E-22 $0.872809 \mathrm{E}-22 \quad 0.886509 \mathrm{E}-22 \quad 0.900100 \mathrm{E}-22 \quad 0.913302 \mathrm{E}-22$ $0.800000 \mathrm{E}+03$ 日. $226000 \mathrm{E}-22 \quad 0.938460 \mathrm{E}-22 \quad 0.950600 \mathrm{E}-22 \quad 0.96270 \mathrm{AE}-22 \quad 0.974500 \mathrm{E}-22$

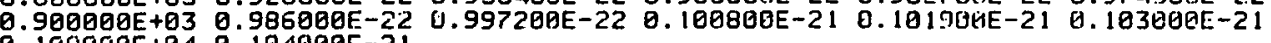
$0.100000 \mathrm{E}+04$ 0.104000E-21

HEP SIGV7 D+6LI $\Rightarrow$ PBE (42.3)+N(2957)

REF 3

$T(J)$ IN KEV SIGVO(J) IN M3/SEC

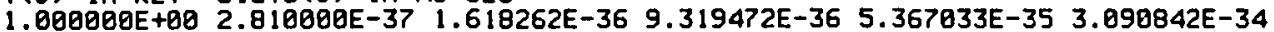

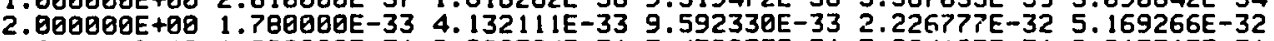
$3.000000 E+00$ 1.200000E-31 $2.029394 E-31 \quad 3.432033 E-31 \quad 5.804127 E-31$ 9.B15715E-31 4. $000000 E+00 \quad 1.660000 E-302.405177 E-30 \quad 3.484872 E-30 \quad 5.049241 E-30 \quad 7.315867 E-30$ 5. .00000E+Q日 1.060000E-29 $1.405214 \mathrm{E}-29$ 1.862856E-29 $2.469539 \mathrm{E}-293.273804 \mathrm{E}-29$

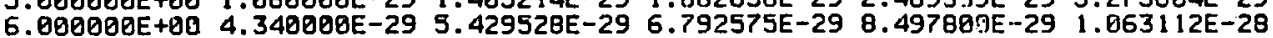
7. QRQ000E+0Q $1.330000 \mathrm{E}-28$ 1.5970 13E-20 $1.917635 \mathrm{E}-28 \quad 2.302625 \mathrm{E}-2 \mathrm{~B} \quad 2.764907 \mathrm{E}-28$

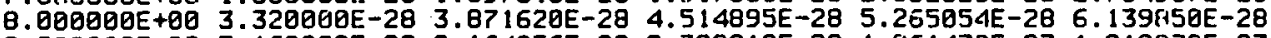

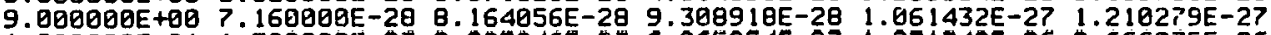
1.000000E+01 1.380000E-27 $2.893246 E-27 \quad 6.065954 E-27 \quad 1.271740 E-26 \quad 2.666375 E-26$ 0.200090E+.02 0.559000E-25 0.032000E-25 0.121200E-240.171400E-24 0.23400日E-24

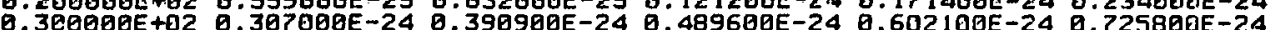

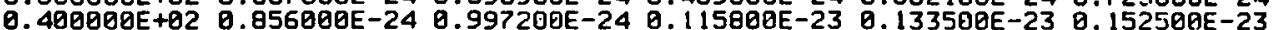
$0.590000 \mathrm{E}+02 \quad 0.172000 \mathrm{E}-23 \quad 0.192400 \mathrm{E}-23 \quad 0.214600 \mathrm{E}-23 \quad 0.2393 \mathrm{AgE}-23 \quad 0.263000 \mathrm{E}-23$

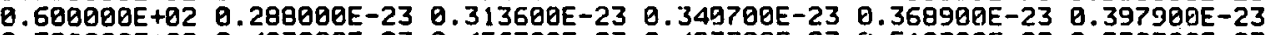
$0.700000 E+02$ 0.427000E-23 $0.456700 \mathrm{E}-23$ 0.487700E-23 $0.519700 \mathrm{E}-23$ e.552300E-23 $\begin{array}{llllll}0.800000 E+02 & 0.585000 E-23 & 0.618000 E-23 & 0.651700 E-23 & 0.686200 E-23 & 0.721600 \mathrm{E}-23\end{array}$

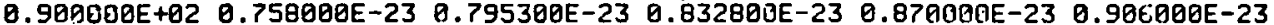
$0.100000 \mathrm{E}+03$ 0.940000E-23 0.129500E-22 0.169300E-22 $0.218 \mathrm{BO0E}-22$ 0.25日800E-22 $0.200900 \mathrm{E}+03$ 0.2860日0E-22 $0.319000 \mathrm{E}-22$ 0.353601IE-22 $0.300100 \mathrm{E}-22$ Q.420405E-22

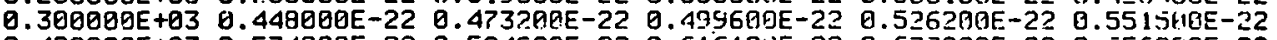

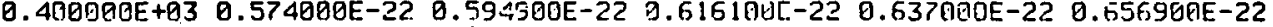

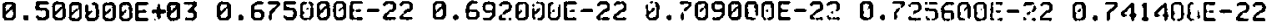

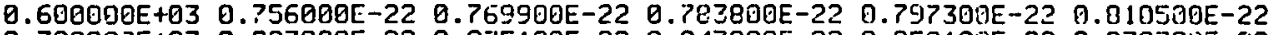

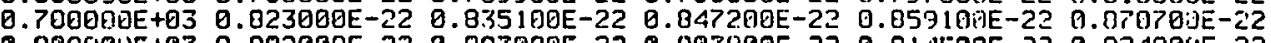
0.800ย9UE+03 0.883ด00E-22 0.093000E-22 0.903900E-22 0.914l:00E-22 0.92499uE-22 
Table I (continued)

T(J) IN KEV SIGVG(J) IN MB/SEC

1.800000E+00 1.820000E-37 $1.053011 E-36 \quad 6.092492 E-36 \quad 3.524986 E-35 \quad 2.039480 E-34$ 2.000000E+00 1.180900E-33 2.743721E-33 6.379670E-33 $1.483396 E-32 \quad 3.449176 \mathrm{E}-32$ $3.000000 \mathrm{E}+00$ B. $020000 \mathrm{E}-32 \quad 1.361302 \mathrm{E}-312.310656 \mathrm{E}-31 \quad 3.922077 \mathrm{E}-31$ 6.657283E-31 $4.000000 \mathrm{E}+00$ 1.130000E-30 1.642864E-30 2.388498E-30 3.472550E-30 5.048610E-30

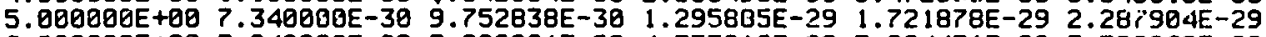
6.000000E+00 3.040000E-29 3.809991E-29 4.775019E-29 5.984471E-29 $7.500269 \mathrm{E}-29$ $7.000000 \mathrm{E}+00$ 9.400000E-29 $1.131924 \mathrm{E}-20 \quad 1.363036 \mathrm{E}-28 \quad 1.641334 \mathrm{E}-201.976454 \mathrm{E}-28$

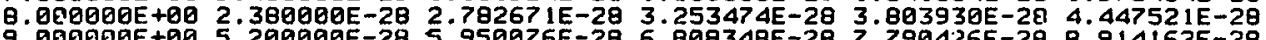

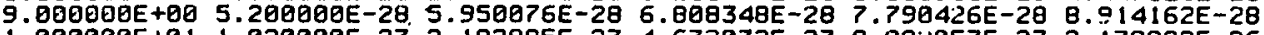

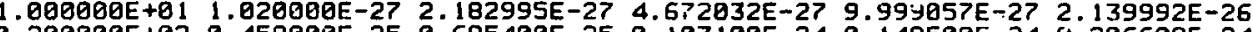
$0.200000 \mathrm{E}+02 \quad 0.458000 \mathrm{E}-25 \quad 0.695400 \mathrm{E}-25 \quad 0.103100 \mathrm{E}-24 \quad 0.140500 \mathrm{E}-24 \quad 0.206680 \mathrm{E}-24$ $0.300000 \mathrm{E}+02$ 0.276000E-24 $0.357800 \mathrm{E}-24 \quad 0.456100 \mathrm{E}-24 \quad 0.5707013 \mathrm{E}-24 \quad 0.700000 \mathrm{E}-24$ $0.400000 \mathrm{E}+02$ 0.840000E-24 $0.995800 \mathrm{E}-24$ 0.117600E-23 $0.137900 \mathrm{E}-23$ 0.160000E-23 $0.500000 \mathrm{E}+02$ 0.183000E-23 $0.207400 \mathrm{E}-23$ 0.234200E-23 $0.263300 \mathrm{E}-23 \quad 0.29410 \mathrm{UE}-23$ $\begin{array}{llllll}0.600000 E+02 & 0.326000 E-23 & 0.359500 E-23 & 0.395500 E-23 & 0.433600 E-2.3 & 0.473400 E-23\end{array}$

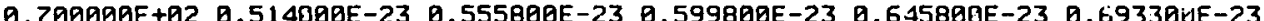
$0.800000 E+02 \quad 0.742000 E-23 \quad 0.79200 \mathrm{EE}-23$ 0.843700E-23 $0.897200 \mathrm{E}-23$ 0.952600E-23 $0.900000 \mathrm{E}+02$ 0.181000E-22 $0.106900 \mathrm{E}-22 \quad 0.112900 \mathrm{E}-22$ 0.11880CE-22 $0.124500 \mathrm{E}-22$

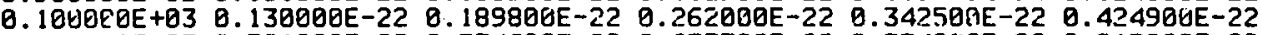
$0.200900 \mathrm{E}+03 \quad 0.501000 \mathrm{E}-22 \quad 0.574600 \mathrm{E}-22 \quad 0.653700 \mathrm{E}-22 \quad 0.734 \mathrm{BU} 0 \mathrm{E}-22 \quad 0.813000 \mathrm{E}-22$

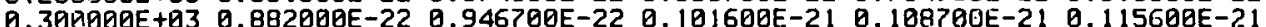
$0.400000 \mathrm{E}+03$ 0.122000E-21 $0.128100 \mathrm{E}-21$ 0.134500E-2 1 0.140900E-21 0.147 100E-21 $0.500000 \mathrm{E}+03$ 0. $153000 \mathrm{E}-21$ 0.158700E-21 $0.154500 \mathrm{E}-21$ 0.170200E-21 $0.175709 \mathrm{E}-21$ $0.600000 \mathrm{E}+03$ 0.181000E-21 $0.186100 \mathrm{E}-21$ 0.191200E-21 0.196300E-21 $0.2013 \mathrm{BDE}-21$ $\begin{array}{lllllll}0.700000 E+03 & 0.206000 E-21 & 0.210600 E-21 & 0.215300 E-21 & 0.219900 E-21 & 0.224500 E-21\end{array}$ $0.890000 \mathrm{E}+03$ 0.229000E-21 $0.233500 \mathrm{E}-21$ 0.237900E-2 1 0.242300E-2i $0.246700 \mathrm{E}-21$ $0.900000 \mathrm{E}+03$ 0.251000E-21 $0.255200 \mathrm{E}-21 \quad 0.259300 \mathrm{E}-21 \quad 0.263300 \mathrm{E}-21 \quad 0.267200 \mathrm{E}-21$ 0.10E0EGE+04 0.271000E-21

AQ9 SIGVS $D+6 L I \Rightarrow P(1618)+A(484)+T(539)$

REF 3

T(J) IN KEV SIGVA(J) IN M3/SEC

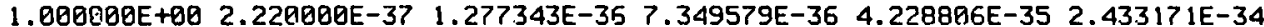
2. $3.000000 \mathrm{C}+00$ 9.280000E-32 $1.568483 \mathrm{E}-312.651015 \mathrm{E}-31$ 4.480607E-31 $7.573157 \mathrm{E}-31$ 4. $000000 \mathrm{E}+00$ 1.280000E-30 $1.852617 \mathrm{~F}-30.2 .68140 \mathrm{IE}-30 \quad 3.88094 \mathrm{PE}-305.617123 \mathrm{EE}-30$

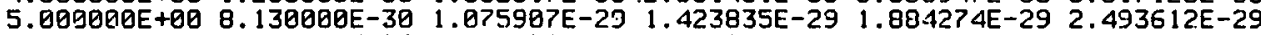
$6.000000 \mathrm{E}+003.300000 \mathrm{E}-29 \quad 4.119178 \mathrm{E}-29 \quad 5.141716 \mathrm{E}-29 \quad 6.418080 \mathrm{E}-29$ 8.011292E-29

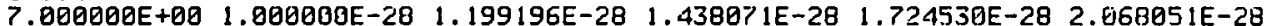
$8.000000 \mathrm{E}+00$ 2.480000E-28 $2.89005 \mathrm{BE}-28 \quad 3.367919 \mathrm{E}-28 \quad 3.924792 \mathrm{E}-28 \quad 4.573743 \mathrm{E}-2 \mathrm{~B}$

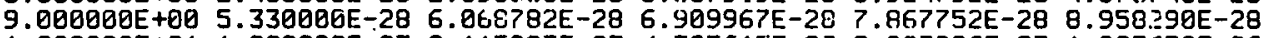
1.000000E+01 1.020000E-27 2.115023E-27 4.385615E-27 9.093006E-27 1.885650E-26

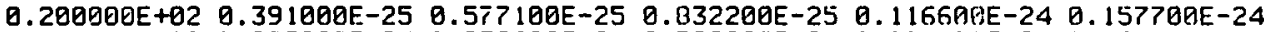

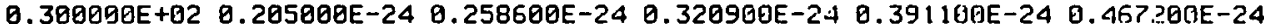
$0.400000 \mathrm{E}+02$ 0.546000E-24 $0.630300 \mathrm{E}-24$ 0.725300E-24 $0.329100 \mathrm{E}-24 \quad 0.930390 \mathrm{E}-24$ $0.500000 \mathrm{E}+02$ 0.105000E-23 $0.116500 \mathrm{E}-23$ 0.128900E-23 $0.142090 \mathrm{E}-23$ 0.155500E-23 $0.60000 \mathrm{EE}+02$ 0.169000E-23 0.182/00E-23 0.197100E-23 0.211900E-23 0.227U00E-23 $0.700000 \mathrm{E}+02$ 0.242000E-23 0.257 $200 \mathrm{E}-23$ 0.272900E-23 $0.209000 \mathrm{E}-23$ 0.30510日E-23

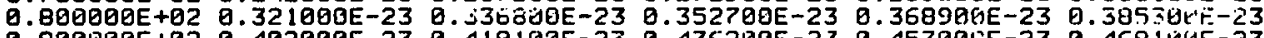

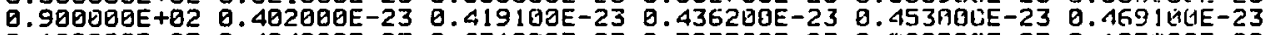
$0.109000 \mathrm{E}+03$ 0. $484000 \mathrm{E}-23$ 0.631200E-23 0.783500E-23 $0.92950 \mathrm{AE}-23$ 0.195600E-22 $0.200000 \mathrm{E}+03$ 0.116000E-22 0.124900E-22 $0.133900 \mathrm{E}-22$ 0.142501E-22 0.150100E-22

9.200000E+03 0.156000E-22 0.160900E-22 $0.166000 \mathrm{E}-2.2$ 月. $17110 \mathrm{BE}-22.0 .175000 \mathrm{E}-22$ $0.490000 \mathrm{E}+03$ 0.180003E-22 0. $183800 \mathrm{E}-22$ 0.187600E-22 0.191700E-22 0.195400E-22

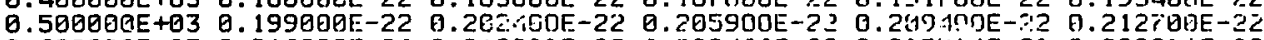

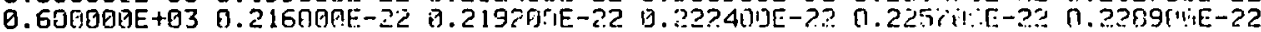

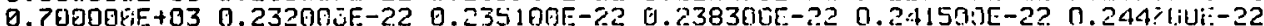

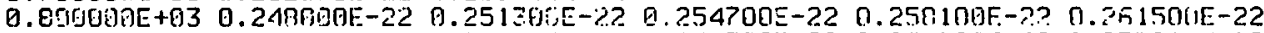
$0.9900 \mathrm{dQE}+03$ 0.2659ค0E-2? U.2. $0.100000 \mathrm{E}+\mathrm{G} 4 \mathrm{G} .201000 \mathrm{E}-22$ 
Table I (continued)

$T$ (J) IN KEV SIGVB (J) IN M3/SEC

1. $000090 \mathrm{E}+00$ 1.450000E-37 $\quad 8.360420 \mathrm{E}-37 \quad 4.820463 \mathrm{E}-36 \quad 2.779393 \mathrm{E}-35 \quad 1.602546 \mathrm{E}-34$ $2.090000 \mathrm{E}+00$ 9.240000E-34 $2.1 .42965 \mathrm{E}-33$ 4.970032E-33 $1.152665 \mathrm{E}-32 \quad 2.673297 \mathrm{E}-32$ 3.000000E+00 6.200000E-32 $1.049577 \mathrm{E}-31$ 1.776795E-31 3.007B78E-31 5.091942E-31 4. $000000 \mathrm{E}+00 \quad 8.620003 \mathrm{E}-31 \quad 1.250116 \mathrm{E}-30 \quad 1.812983 \mathrm{E}-30 \quad 2.629285 \mathrm{E}-30 \quad 3.813127 \mathrm{E}-30$ $5.000000 \mathrm{E}+00 \quad 5.530000 \mathrm{E}-3 \mathrm{~B} \quad 3.328269 \mathrm{E}-30 \quad 9.711314 \mathrm{E}-30 \quad 1.286929 \mathrm{E}-29 \quad 1.705$ 120E-29 $6.000000 \mathrm{E}+00 \quad 2.260000 \mathrm{E}-29 \quad 2.826880 \mathrm{E}-29 \quad 3.535956 \mathrm{E}-23 \quad 4.422893 \mathrm{E}-29 \quad 5.532503 \mathrm{E}-29$ $7.800000 \mathrm{E}+00$ 6.920000E-29 $8.311769 \mathrm{E}-29$ 9.383468E-29 $1.199140 \mathrm{E}-28 \quad 1.440316 \mathrm{E}-28$ 8.090000E+0日 $1.730000 \mathrm{E}-28 \quad 2.018417 \mathrm{E}-28 \quad 2.354919 \mathrm{E}-28 \quad 2.747522 \mathrm{E}-28 \quad 3.205577 \mathrm{E}-28$ $9.000400 E+00 \quad 3.740000 \mathrm{E}-28 \quad 4.268201 \mathrm{E}-23 \quad 4.871000 \mathrm{E}-28 \quad 5.550935 \mathrm{E}-28 \quad 6.344924 \mathrm{E}-28$ 1.

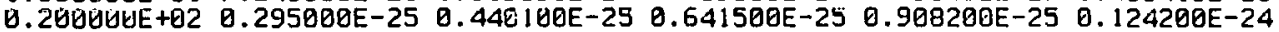
$0.300000 \mathrm{E}+02$ 0.163000E-24 $0.287700 \mathrm{E}-24 \quad 0.260300 \mathrm{E}-24 \quad 0.320400 \mathrm{E}-24 \quad 0.386800 \mathrm{E}-24$ $0.400000 E+02$ 0.457000E-24 0.533700E-24 $0.621200 E-24 \quad 0.718300 E-24 \quad 0.822300 E-24$

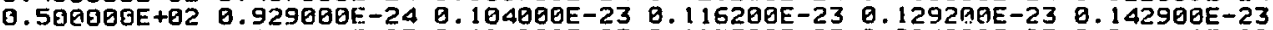

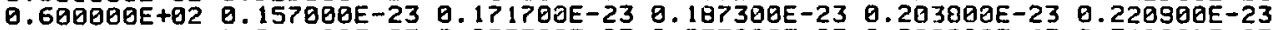

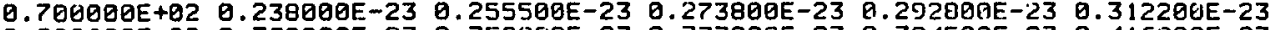
0. QDQDQQE +02 $0.332000 \mathrm{E}-23$ 0.352200E-23 $0.373000 \mathrm{E}-23$ 0.394509E-23 $0.416900 \mathrm{E}-23$ $0.900000 \mathrm{E}+02$ 0.449000E-23 $0.463 \mathrm{BO \theta E}-23 \quad 0.480000 \mathrm{E}-23 \quad 0.512200 \mathrm{E}-23 \quad 0.536000 \mathrm{E}-23$ $0.100000 \mathrm{E}+03$ 0.559000E-23 $0.81050 \mathrm{EE}-23$ 0.111000E-22 $0.144100 \mathrm{E}-22 \quad 0.178000 \mathrm{E}-22$ $0.200000 \mathrm{E}+03$ 0.2 $10000 \mathrm{E}-22$ 0.241700E-22 $0.275700 \mathrm{E}-22 \quad 0.310600 \mathrm{E}-22 \quad 0.344200 \mathrm{E}-22$ 0.300000E+03 0.374900E-22 $0.402000 \mathrm{E}-22 \quad 0.431000 \mathrm{E}-22 \quad 0.462200 \mathrm{E}-22 \quad 0.491500 \mathrm{E}-22$

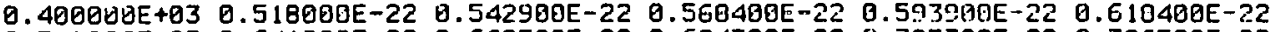
$0.509000 \mathrm{E}+03$ 0.641000E-22 $0.662500 \mathrm{E}-22 \quad 0.684300 \mathrm{E}-22 \quad 0.705700 \mathrm{E}-22 \quad 0.726590 \mathrm{E}-22$ Q. $600000 \mathrm{E}+03$ 0.746000E-22 $0.764800 \mathrm{E}-22 \quad 0.783700 \mathrm{E}-22 \quad 0.802300 \mathrm{E}-22 \quad 0.820500 \mathrm{E}-22$ 0.700000E+03 0.838000E-22 $0.855000 \mathrm{E} \cdots 22$ 0.072006E-22 $0.880000 \mathrm{E}-22$ 0.905200E-22 0.804000E+03 0.921060E-22 $0.936400 \mathrm{E}-22 \quad 0.351500 \mathrm{E}-22 \quad 0.966400 \mathrm{E}-22$ 0.980900E-22 0.900000E+03 0.995000E-22 0.100900E-21 0.102200E-21 0.103500E-21 0.104900E-21 $0.100000 \mathrm{E}+04$ 0.106000E-21

A11 SIGVB D+6L.I $\Rightarrow 3 H E(378)+A(284)+N(1134)$

$T(J)$ IN KEV SIGVC ( $J)$ IN M3/SEC

O. $100000 E+01$ 0.000000E+00 0.000000E+00 0.000000E+00 0.000000E+00 0.000000E+00

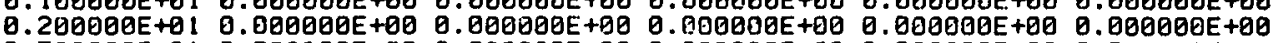
$0.300000 \mathrm{E}+01$ 0.000000E+00 0.000000E+00 0.000000E+00 0.000000E+00 0.000000E+00 $0.400000 \mathrm{E}+01 \quad 0.000000 \mathrm{E}+00 \quad 0.000000 \mathrm{\theta}+00 \quad 0.000000 \mathrm{E}+00 \quad 0.000000 \mathrm{E}+00 \quad 0.000000 \mathrm{E}+00$

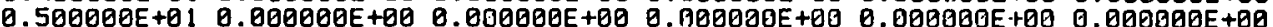
$0.609000 E+01 \cdot 0.000000 E+00$ 0.000000E+00 0.000000E+E9 0.000000E+00 0.000000E+00

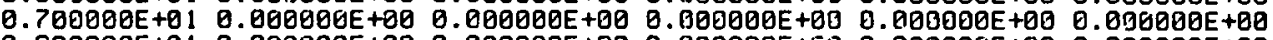
$0.800000 \mathrm{E}+01$ 0.000000E+00 0.000000E+00 0.000000E+00 0.000000E+00 0.000000E+00

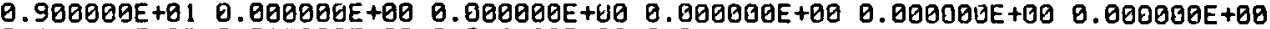
B. $100000 \mathrm{E}+02$ B. $090000 \mathrm{E}$ +

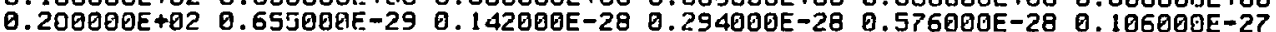

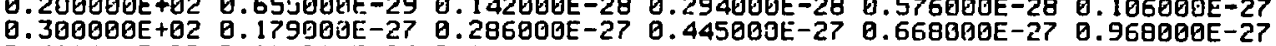
$0.400000 \mathrm{E}+02$ 0.135000E-26 $0.184000 \mathrm{E}-26 \quad 0.250000 \mathrm{E}-26 \quad 0.335000 \mathrm{E}-26 \quad 0.442009 \mathrm{E}-26$ $0.500000 E+02$ 0.569000E-26 $0.721000 \mathrm{E}-26$ 0.909000E-26 $0.114000 \mathrm{E}-25$ 0.140000E-2.5 $0.600000 \mathrm{E}+92 \quad 0.171000 \mathrm{E}-25 \quad 0.206000 \mathrm{E}-25 \quad 0.248000 \mathrm{E}-25 \quad 0.296000 \mathrm{E}-25 \quad 0.351000 \mathrm{E}-25$ $0.700000 \mathrm{E}+02$ 0.411000E-25 0.478000E-25 0.555000E-25 0.649009E-25 0.734999E-25 $0.800000 \mathrm{E}+02$ 0.936000E-25 0.947000E-25 0.107000E-24 0.120000E-24 0.135000E-24 $0.900000 \mathrm{E}+02$ 0.151000E-24 0.169000E-24 0.187000E-2.4 ค. $297000 \mathrm{E}-24$ 0.227000E-24 $0.100000 E+03$ 0.24PUDUE-24 0.515000[-24 0.353000E-24 0.15TE00E-2J 0.23400EE-23 $0.2009 E 0 E+03 \quad 0.315000 E-23 \quad 0.403990 E-23 \quad 0.508000 E-23 \quad 0.627$ Ejd0E-23 $0.751000 E-23$

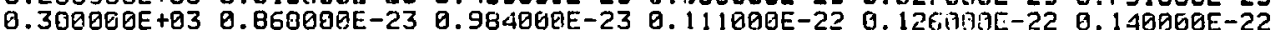

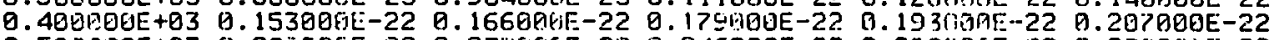

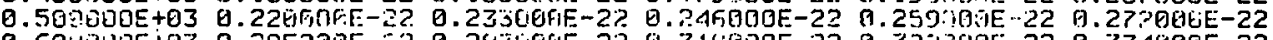

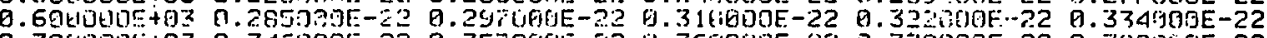

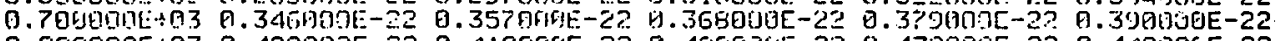

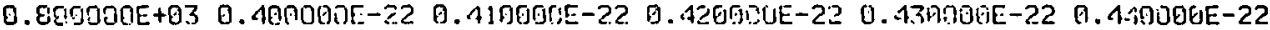

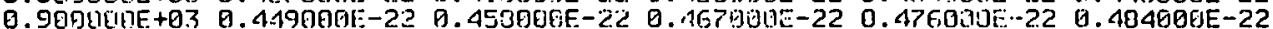

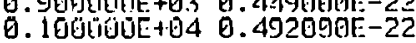


Table I (continued)

T(J) IN KEV SIGVD(J) IN MB/SEC

1. 2.000000E+00 4.012000E-38 8.590000E-37 1. 091000E-35 $9.287000 E-35$ 5.773008E-34 $3.000000 \mathrm{E}+0 \mathrm{~B} \quad 2.792000 \mathrm{E}-33 \quad 1.102000 \mathrm{E}-32 \quad 3.682000 \mathrm{E}-32 \quad 1.070000 \mathrm{E}-31 \quad 2.769080 \mathrm{E}-31$

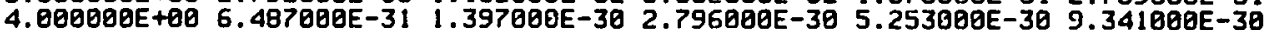
5. $000000 \mathrm{E}+00$ 1.583000E-29 $2.569000 \mathrm{E}-29$ 4.016000E-29 $6.070000 \mathrm{E}-29$ 8.902000E-29 6. .000000E+Q日 $1.271000 \mathrm{E}-28$ 1.771000E-28 $2.414000 \mathrm{E}-28 \quad 3.227900 \mathrm{E}-28$ 4.235000E-28

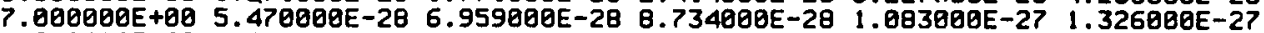
8. $.000000 \mathrm{E}+00 \quad 1.608000 \mathrm{E}-27 \quad 1.931800 \mathrm{E}-27 \quad 2.298000 \mathrm{E}-27 \quad 2.712000 \mathrm{E}-27 \quad 3.176000 \mathrm{E}-27$ 9. 1. D00009E+01 $7.167000 E-27 \quad 1.955000 E-26 \quad 4.092000 E-26 \quad 7.300000 E-26 \quad 1.175000 E-25$ $2.000000 \mathrm{E}+01$ 1.756000E-25 $2.481000 \mathrm{E}-25$ 3.359000E-25 $4.393000 \mathrm{E}-25$ 5.588000E-25

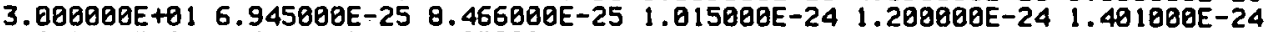
4. B00000E+ 11 1.618000E-24 $1.850000 \mathrm{E}-24 \quad 2.098000 \mathrm{E}-24 \quad 2.361000 \mathrm{E}-24 \quad 2.639000 \mathrm{E}-24$ $\begin{array}{llllll}5 . \theta 00000 E+01 & 2.9300 \theta 0 E-24 & 3.236000 E-24 & 3.554000 E-24 & 3.885000 E-24 & 4.227000 E-24\end{array}$ 6. 000000E+01. 4.581000E-24 4.946000E-24 5.321000E-24 5.706000E-24 6.100000E-24 $7.000000 E+016.502000 E-24 \quad 6.913000 E-24 \quad 7.331000 E-24 \quad 7.756000 E-24 \quad 8.180000 E-24$ B. Q00000E+Q 1 8.62600日E-24 9.070000E-24 9.519000E-24 9.973000E-24 1.043000E-23 $\begin{array}{llllll}9.000000 E+01 & 1.090000 E-23 & 1.136000 E-23 & 1.183000 E-23 & 1.231900 E-23 & 1.279000 E-23\end{array}$ 1. $008000 \mathrm{E}+02$ 1.327000E-23 $1.819000 \mathrm{E}-23 \quad 2.322000 \mathrm{E}-23 \quad 2.830000 \mathrm{E}-23 \quad 3.338000 \mathrm{E}-23$ $2.000000 \mathrm{t}+02 \quad 3.845000 \mathrm{E}-23 \quad 4.351000 \mathrm{E}-23 \quad 4.857000 \mathrm{E}-23 \quad 5.360000 \mathrm{E}-23 \quad 5.851000 \mathrm{E}-23$ 3. 4.000000E+R2 $8.763000 E-23 \quad 9.222000 E-23 \quad 9.671000 E-23 \quad 1.811000 E-22 \quad 1.054080 E-22$ 5. 000000E+02 $1.096000 \mathrm{E}-22$ 1.137000E-22 $1.176000 \mathrm{E}-22$ 1.2.15000E-22 $1.252000 \mathrm{E}-22$

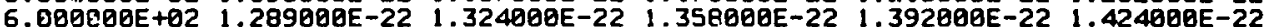
7.000000E+02 $1.455000 \mathrm{E}-22$ 1.485000E-22 $1.514000 \mathrm{E}-22$ 1.543000E-22 $1.570000 \mathrm{E}-22$

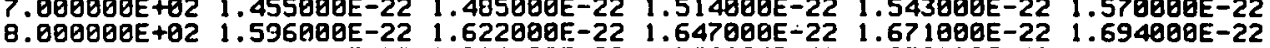

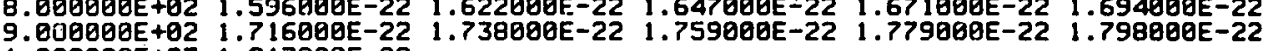
$1.000000 \mathrm{E}+03$ 1.817000E-22

A13 SIGVD P+6LI $\Rightarrow 3$ HE (2298)+A(1724)

REF 4

T(J) IN KEV SIGVE (J) IN M3/SEC

1.000000E+09 $6.248073 E-42 \quad 1.236762 E-40 \quad 1.523416 E-39 \quad 1.189332 E-38 \quad 8.753124 E-38$

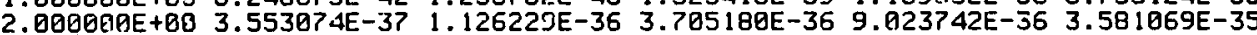

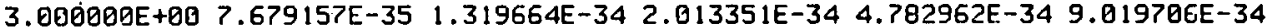

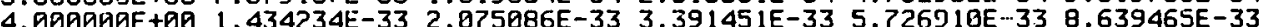
5.000000E+00 1.212912E-32 1.6E7G53E-32 2.573613E-3? 3.664982E-32 4.961762E-32 6.000000E+00 6.463952E-32 9.021606E-32 $1.221718 \mathrm{E}-31 \quad 1.597095 \mathrm{E}-31 \quad 2.030689 \mathrm{E}-31$ $7.090000 \mathrm{E}+00 \quad 2.623214 \mathrm{E}-31 \quad 3.395038 \mathrm{E}-31$ 4.292832E-31 $5.316594 \mathrm{E}-31$ 6.553853E-31 $8.000000 \mathrm{E}+00$ 8.195594E-31 $1.00871 \mathrm{IE}-30 \quad 1.222840 \mathrm{E}-30 \quad 1.462272 \mathrm{E}-301.778353 \mathrm{E}-30$ 9. 1. 2. $000000 \mathrm{E}+01.5 .448750 \mathrm{E}-28 \quad 9.325781 \mathrm{E}-28$ 1.537726E-27 $2.334615 \mathrm{E}-27 \quad 3.484482 \mathrm{E}-27$

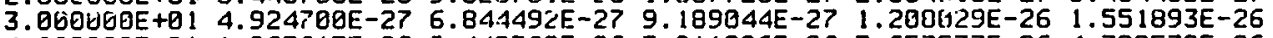
4. $000000 \mathrm{E}+01 \quad 1.963613 \mathrm{E}-26 \quad 2.449768 \mathrm{E}-26 \quad 3.011926 \mathrm{E}-26 \quad 3.657577 \mathrm{E}-26 \quad 4.382579 \mathrm{E}-26$

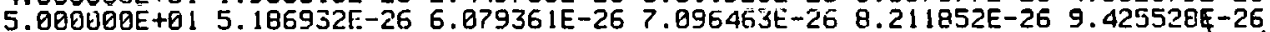
6. $000000 E+01$ 1. $073749 E-25$ 1.216098E-25 $1.370958 E-25 \quad 1.535747 E-25 \quad 1.711267 E-25$ 7. $000000 \mathrm{E}+011.900601 \mathrm{E}-252.104334 \mathrm{E}-25 \quad 2.320871 \mathrm{E}-252.550213 \mathrm{E}-252.792231 \mathrm{E}-25$ 8.000000E+01 3.046649E-25 3.313690E-25 3.593355E-25 3.885653E-25 4.192072E-25 $9.000000 E+Q 1 \quad 4.511682 E-25 \quad 4.844483 E-25 \quad 5.190474 E-25 \quad 5.549657 E-25 \quad 5.922030 E-25$ 1. $909090 \mathrm{E}+02$ 6.397594E-25 $1.087775 \mathrm{E}-24$ 1.667903E-24 2.36195EE-24 $3.168375 \mathrm{E}-24$ $2.000000 \mathrm{E}+02$ 4.073524E-24 5.076392E-24 6.163758E-24 $7.340049 E-24$ 8.589610E-24

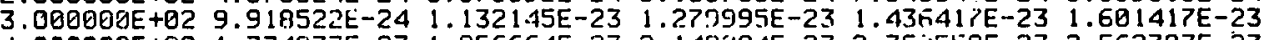
4. $000900 E+02$ 1. $7>4833 E-23$ 1.9.96664E-23 $2.149294 \mathrm{E}-23 \quad 2.351559 \mathrm{E}-232.562383 \mathrm{E}-23$

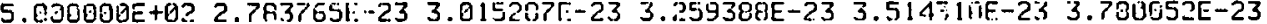

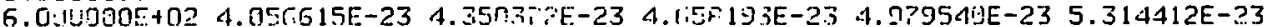

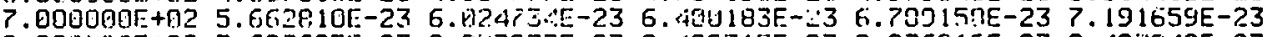

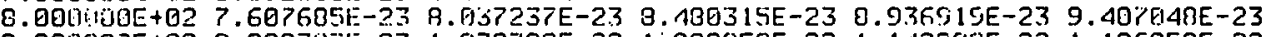

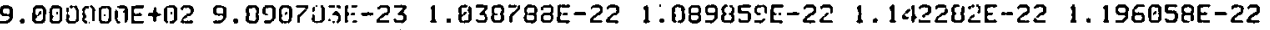

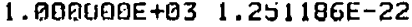


Table I (continued)

T(J) IN KEV SIGVF (J) IN MB/SEC

$1.000000 \mathrm{E}+00$ 2.225530E-48 B.846070E-45 3.204950E-42 2.622990E-40 B. $118990 \mathrm{E}-39$

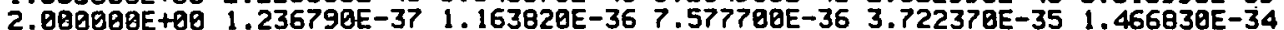

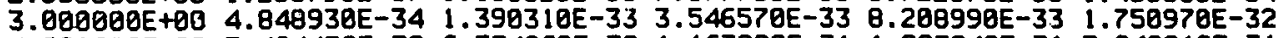
$4.000000 E+00 \quad 3.484450 E-32 \quad 6.534060 E-32 \quad 1.163990 E-31 \quad 1.983040 E-31 \quad 3.248910 E-31$ $5.000000 \mathrm{t}+0 \mathrm{~B} \quad 5.142650 \mathrm{E}-317.895550 \mathrm{E}-31 \quad 1.179700 \mathrm{E}-30 \quad 1.720390 \mathrm{E}-302.454430 \mathrm{E}-30$ 6. BODOODE+ 00 3.433600E-30 4.718750E-30 6.3B1290E-30 $8.504340 E-30$ 1.118410E-29

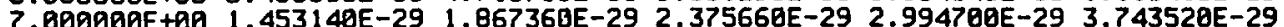

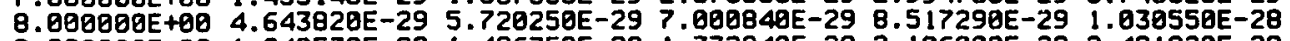

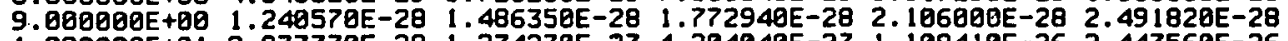
$1.000000 \mathrm{E}+012.937330 \mathrm{E}-2 \mathrm{\theta} \quad 1.274270 \mathrm{E}-27 \quad 4.204040 \mathrm{E}-27 \quad 1.108410 \mathrm{E}-26 \quad 2.447560 \mathrm{E}-26$

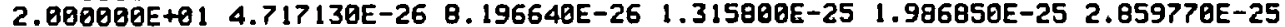

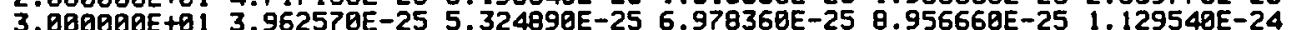

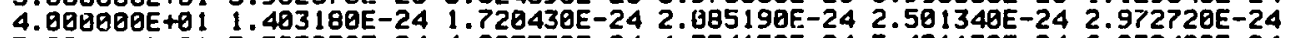
5.000000E+QI $3.503030 E-24 \quad 4.095750 E-24 \quad 4.754150 E-24 \quad 5.481170 E-24 \quad 6.279420 E-24$

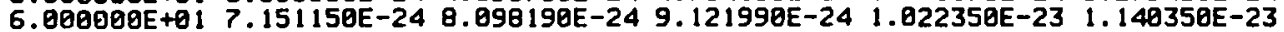
$7.000000 \mathrm{E}+011.266190 \mathrm{E}-23 \quad 1.399880 \mathrm{E}-23 \quad 1.541340 \mathrm{E}-23 \quad 1.690480 \mathrm{E}-23 \quad 1.847 \quad 190 \mathrm{E}-23$

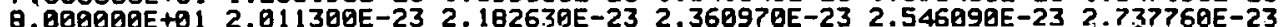

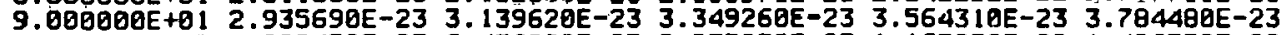
$1.000000 \mathrm{t}+02$ 4.009450E-23 $6.456900 \mathrm{E}-23$ 9.073050E-23 $1.165030 \mathrm{E}-22$ 1.406570E-22 $2.000000 \mathrm{E}+021.626120 \mathrm{E}-22 \quad 1.821960 \mathrm{E}-22 \quad 1.994730 \mathrm{E}-22 \quad 2.14626 \mathrm{EE}-22 \quad 2.278820 \mathrm{E}-22$

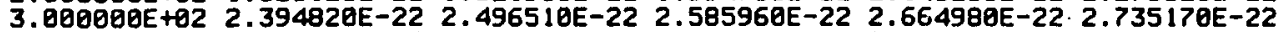
$4.000000 E+02$ 2.797060E-22 2.054220E-22 2.905210E-22 2.9916T0E-22 2.994290E-22 $5.000000 \mathrm{t}+02$ 3.033660E-22 3.070270E-22 3.104550E-22 $3.136830 \mathrm{E}-22$ 3.167430E-22

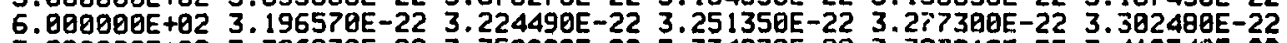
7. 000000E+02 $3.326970 \mathrm{E}-22 \quad 3.35 \mathrm{~B} \theta \mathrm{BE}-22 \quad 3.3742 \mathrm{PQE}-22 \quad 3.397210 \mathrm{E}-22 \quad 3.419740 \mathrm{E}-22$ 8.000000E+02 $3.441910 E-22 \quad 3.463760 E-22 \quad 3.495300 E-22 \quad 3.506570 E-22 \quad 3.527570 E-22$ $9.080000 \mathrm{E}+02$ 3.548330E-22 $3.568 \theta 40 \mathrm{E}-22 \quad 3.589128 \mathrm{E}-22 \quad 3.6891 \mathrm{B \theta E}-22 \quad 3.629000 \mathrm{E}-22$ 1. $.00000 \mathrm{\theta}+03$ 3.64859日E-22

$A 15$ SIGVF $P+11 B \rightarrow A(28 B 8)+A(2888)+A(2888)$

REF 6

T(J) IN KEV SICVG(J) IN MB/SEC

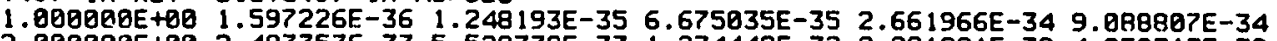
2. .00000E+ 00 2.487753E-33 5.528378E-33 1.234449E-32 2.291921E-32 4.950713E-32 $3.000000 E+00$ 8.734842E-32 $1.364431 E-31 \quad 1.967911 E-31 \quad 3.200650 E-31 \quad 4.900010 E-31$ $4.000900 E+00$ 6.964105E-31 $9.392942 E-31 \quad 1.289958 E-301.791615 E-30 \quad 2.388674 E-30$

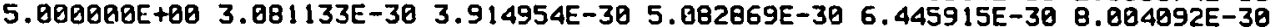
$6.000000 \mathrm{E}+00$ 9.757399E-3日 $1.203218 \mathrm{E}-29$ 1.466813E-29 $1.763771 \mathrm{E}-292.094093 \mathrm{E}-29$

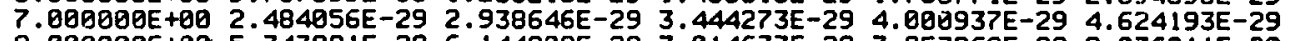
8.000000E+00 5.347981E-29 6.144808E-29 7.014673E-29 7.957968E-29 9.0362IIE-29 $9.000000 \mathrm{E}+00 \quad 1.821094 \mathrm{E}-28 \quad 1.148215 \mathrm{E}-28 \quad 1.204984 \mathrm{E}-28 \quad 1.435001 \mathrm{E}-20 \quad 1.600425 \mathrm{E}-28$ $\begin{array}{llllllll}1.000000 E+01 & 1.777 & 143 E-28 & 4.416189 E-28 & 9.105077 E-28 & 1.659410 E-27 & 2.732166 E-27\end{array}$ $2.000000 \mathrm{E}+01 \quad 4.201040 \mathrm{E}-27 \quad 6.077516 \mathrm{E}-27 \quad 8.462629 \mathrm{E}-27 \quad 1.132300 \mathrm{E}-26 \quad 1.4783 \mathrm{~B} 6 \mathrm{E}-26$

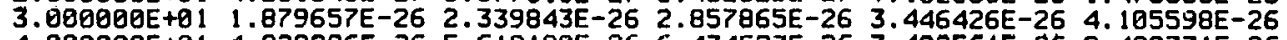
4. $000000 \mathrm{E}+01$ 4.829996E-26 $5.618190 \mathrm{E}-26$ 6.474523E-26 $7.402561 \mathrm{E}-26$ 8.490331E-26 $5.000000 \mathrm{E}+81 \quad 9.467834 \mathrm{E}-26 \quad 1.060535 \mathrm{E}-25 \quad 1.181403 \mathrm{E}-25 \quad 1.309305 \mathrm{E}-25 \quad 1.444248 \mathrm{E}-25$ $6.000000 \mathrm{E}+01 \quad 1.586208 \mathrm{E}-25 \quad 1.733935 \mathrm{E}-25 \quad 1.888047 \mathrm{E}-25 \quad 2 . \dot{0} 48651 \mathrm{E}-25 \quad 2.215747 \mathrm{E}-25$ $7.090000 \mathrm{E}+012.387727 \mathrm{E}-25 \quad 2.564286 \mathrm{E}-25 \quad 2.746254 \mathrm{E}-25 \quad 2.933633 \mathrm{E}-25 \quad 3.126422 \mathrm{E}-25$ 8.00Q日000E+Q1 $3.324621 \mathrm{E}-25 \quad 3.528230 \mathrm{E}-25 \quad 3.737250 \mathrm{E}-25 \quad 3.951657 \mathrm{E}-25$ 4. $167800 \mathrm{E}-25$ 9.0G0000E+Qi $4.387963 E-25$ 4.612144E-25 4.840345E-25 5.072565E-25 5.308803E-25

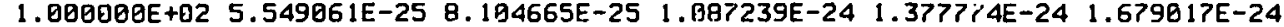
$2.000000 \mathrm{E}+02 \quad 1.991803 \mathrm{E}-24 \quad 2.31744$ AE $-24 \quad 2.661216 \mathrm{E}-24 \quad 3.023604 \mathrm{E}-24 \quad 3.439045 \mathrm{E}-24$

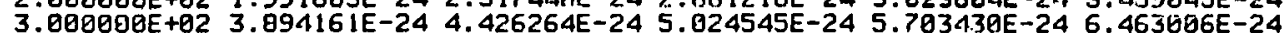

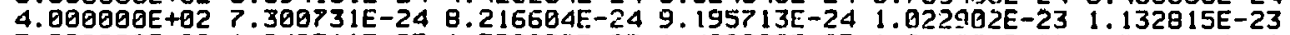
5. $000000 \mathrm{E}+02 \quad 1.249311 \mathrm{E}-23 \quad 1.370 B 52 \mathrm{E}-23 \quad 1.490990 \mathrm{E}-23 \quad 1.614 .374 \mathrm{E}-23 \quad 1.741005 \mathrm{~F}-23$

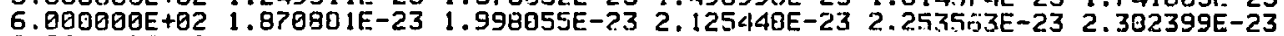

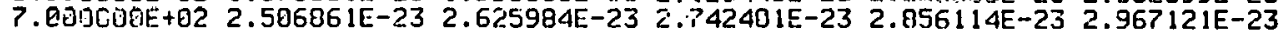

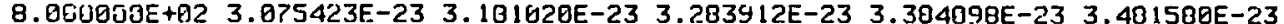
$9.000000 \mathrm{E}+02$ 3.576356E-23 3.668427E-23 3.757793E-23 3.0.11.15.1E-23 3.928410E-23 $1.000060 \mathrm{E}+03$ 4.009ة61E-23 
Table I (continued)

$T(J)$ IN KEV SIGUH(J) IN MB/SEC

$1.000000 E+002.174972 E-37 \quad 2.634161 E-36 \quad 2.073949 E-35 \quad 1.131411 E-34 \quad 5.433681 E-34$ $2.000000 \mathrm{E}+001.821460 \mathrm{E}-33 \quad 4.758154 \mathrm{E}-33 \quad 1.282475 \mathrm{E}-32 \quad 2.683084 \mathrm{E}-32 \quad 7.564635 \mathrm{E}-32$ $3.090090 \mathrm{E}+00$ 1. $489579 \mathrm{E}-312.440654 \mathrm{E}-31 \quad 3.636690 \mathrm{E}-31$ 6.942409E-31 $1.176799 \mathrm{E}-3 \mathrm{~B}$ $4.000000 \mathrm{E}+00 \quad 1.774406 \mathrm{E}-302.487063 \mathrm{E}-303.688020 \mathrm{E}-30 \quad 5.606369 \mathrm{E}-30 \quad 7.949003 \mathrm{E}-30$ $5.000000 \mathrm{E}+00$ 1. $071396 \mathrm{E}-29 \mathrm{1} .422672 \mathrm{E}-29$ 1.983968E-29 $2.657708 \mathrm{E}-29$ 3.443891E-29

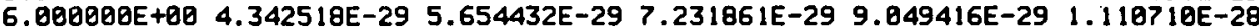
$\begin{array}{lllllll}7.000000 E+00 & 1.370213 E-28 & 1.689093 E-28 & 2.051977 E-28 & 2.458864 E-28 & 2.930218 E-28\end{array}$

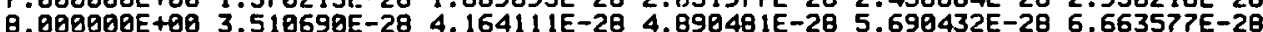
$9.000000 \mathrm{E}+00$ ?.747633E-28 $8.942599 \mathrm{E}-28$ 1.024848E-27 $1.174525 \mathrm{E}-27 \quad 1.342435 \mathrm{E}-27$ $\begin{array}{llllll}1.000000 \mathrm{E}+01 & 1.525945 \mathrm{E}-27 & 4.66325 \mathrm{EE}-27 & 1.132609 \mathrm{E}-26 & 2.353689 \mathrm{E}-26 & 4.355455 \mathrm{E}-26\end{array}$ $2.000000 \mathrm{E}+01 \quad 7.417354 \mathrm{E}-26 \quad 1.163519 \mathrm{E}-25 \quad 1.750501 \mathrm{E}-25 \quad 2.487380 \mathrm{E}-25 \quad 3.449296 \mathrm{E}-25$

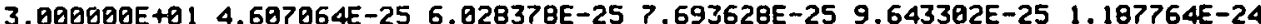
4. 800000E+ 11 1.442273E-24 $1.729290 E-24 \quad 2.048091 E-242.402415 E-242.789122 E-24$

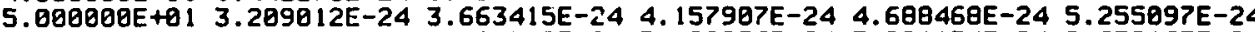
6.000000E to 1 5.857795E-24 6.5008j0E-24 7.182056E-24 7.901174E-24 8.65日163E-24

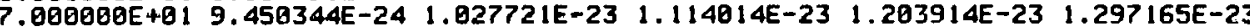
B. QQDQDQE + 1 1 $393212 E-23 \quad 1.492506 E-23 \quad 1.595045 E-23 \quad 1.700836 E-23 \quad 1.818690 E-23$ 9.000000E+01 $1.924098 \mathrm{E}-23$ 2.041062E-23 2.161502E-23 $2.285657 \mathrm{E}-23 \quad 2.413287 \mathrm{E}-23$ $1.000000 \mathrm{E}+02$ 2.544472E-23 $4.016212 \mathrm{E}-23 \quad 5.741862 \mathrm{E}-23 \quad 7.674253 \mathrm{E}-23 \quad 9.775125 \mathrm{E}-23$ $2.000000 \mathrm{E}+02$ 1.201410E-22 $1.437662 \mathrm{E}-22$ 1.677896E-22 $1.92553 \mathrm{BE}-22$ 2.178501E-22 3. $000000 \mathrm{E}+02 \quad 2.437595 \mathrm{E}-22 \quad 2.699090 \mathrm{E}-22 \quad 2.964067 \mathrm{E}-22 \quad 3.226948 \mathrm{E}-22 \quad 3.497702 \mathrm{E}-22$ 4. .日QD日BE +02 3.747254E-22 4.005504E-22 $4.260933 \mathrm{E}-22 \quad 4.512139 \mathrm{E}-22 \quad 4.760640 \mathrm{E}-22$ 5.

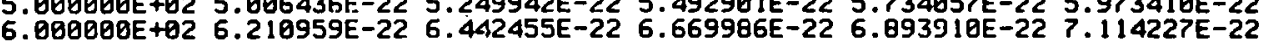
7.000000E+02 $7.33227 \theta E-22$ 7.54B317E-22 $7.761651 E-22$ 7.972280E-22 8. 180204E-22 8.000000E+02 8.385423E-22 8.587937E-22 8.787745E-22 8.98484BE-22 $9.179246 \mathrm{E}-22$ 9. 1. $000000 \mathrm{E}+03$ 1.028883E-21

$A 17$ SIGVH $D+7 L I \Rightarrow N(18082)+A(2521)+A(2521)$

REF 5

T(J) IN KEV SIGVI(J) IN M3/SEC

$1.000000 \mathrm{E}+00$ 5.843883E-39 9.208190E-38 9.178436E-37 $6.063819 \mathrm{E}-36 \quad 3.640056 \mathrm{E}-35$

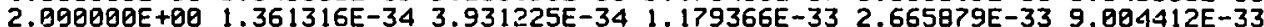

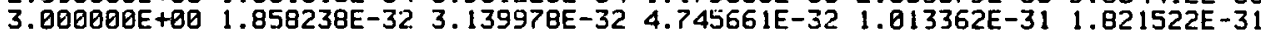
4. . $5.000900 \mathrm{E}+002.052868 \mathrm{E}-302.796164 \mathrm{E}-304.087234 \mathrm{E}-305.659908 \mathrm{E}-307.514187 \mathrm{E}-38$ 6. $000000 E+00$ 9.65007 IE-30 $1.301074 E-29 \quad 1.715299 E-29 \quad 2.197254 E-29 \quad 2.747739 E-29$ 7. $000009 \mathrm{E}+003.475617 \mathrm{E}-294.401550 \mathrm{E}-29 \quad 5.469231 \mathrm{E}-29$ 6.678562E-29 8.11258日E-29

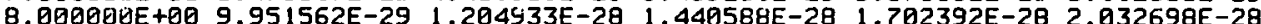
$9.020000 \mathrm{E}+0 \mathrm{Q}$ 2.495023E-28 $2.819368 \mathrm{E}-28 \quad 3.275733 \mathrm{E}-2 \mathrm{~B} \quad 3.814749 \mathrm{E}-28 \quad 4.432066 \mathrm{E}-28$ $1.000000 \mathrm{E}+01$ 5.114397E-28 $1.764293 \mathrm{E}-27 \quad 4.791652 \mathrm{E}-27$ 1.082767E-26 $2.169783 \mathrm{E}-26$ $2.000000 \mathrm{E}+01$ 3.933637E-26 $6.473732 \mathrm{E}-26 \quad 1.026761 \mathrm{E}-25 \quad 1.516945 \mathrm{E}-25 \quad 2.192125 \mathrm{E}-25$

$3.000000 \mathrm{E}+013.023483 \mathrm{E}-25 \quad 4.091503 \mathrm{E}-25 \quad 5.372871 \mathrm{E}-25 \quad 6.927316 \mathrm{E}-25 \quad 8.755184 \mathrm{E}-35$

4. .00000E+Q1 $1.088599 E-24 \quad 1.333691 \mathrm{E}-24 \quad 1.613379 \mathrm{E}-24 \quad 1.932033 \mathrm{E}-242.287236 \mathrm{E}-24$ 5.000900E+01 2.678908E-24 3.109562E-24 3.588483E-24 4.100883E-24 4.670751E-24 6. $000000 \mathrm{E}+01$ 5.274119E-24 5.929578E-24 6.531921E-24 7.380252E-24 8.174569E-24

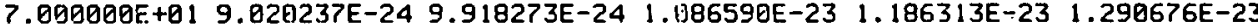

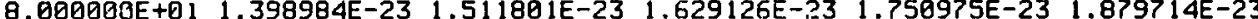
$9.000030 \mathrm{E}+012.013863 \mathrm{E}-23 \quad 2.153422 \mathrm{E}-23 \quad 2.298392 \mathrm{E}-23 \quad 2.448771 \mathrm{E}-23 \quad 2.604561 \mathrm{E}-23$ $1.000000 \mathrm{E}+022.765762 \mathrm{E}-23 \quad 4.633491 \mathrm{E}-23 \quad 6.945232 \mathrm{E}-23$ 9.670615E-23 $1.273136 \mathrm{E}-22$ $2.000000 \mathrm{E}+02 \quad 1.610334 \mathrm{E}-22 \quad 1.977490 \mathrm{E}-22 \quad 2.36459$ !E-22 $2.775-156 \mathrm{E}-22 \quad 3.1950 \mathrm{~T} 2 \mathrm{E}-22$

3.000900E+02 3.629116E-22 4.072257E-22 4.525040E-22 $4.986366 \mathrm{E}-22 \quad 5.453213 \mathrm{E}-22$

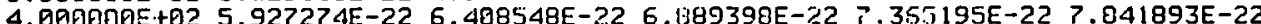

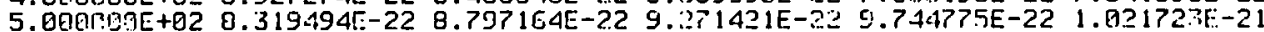

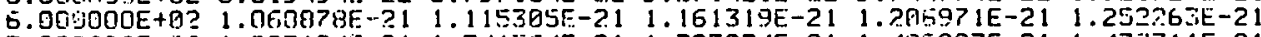

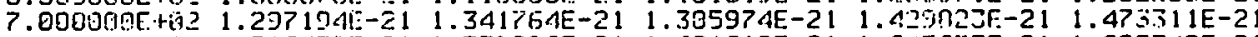

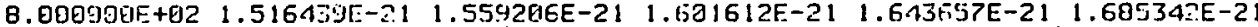

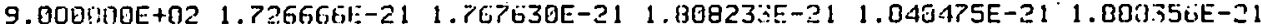
1. 000000E+03 1.92707フE-21 
Table I (continued)

$T(J)$ IN KEV SIGVJ(J) IN MB/SEC

1. $000000 E+00$ 1.525961E-50 $1.161848 E-48 \quad 5.5 B 2734 E-47 \quad 1.197673 E-45 \quad 3.729008 E-44$ 2. $000000 \mathrm{E}+00$ 2.061344E-43 $1.309807 \mathrm{E}-42 \quad 6.623920 \mathrm{E}-42 \quad 3.292113 \mathrm{E}-41 \quad 3.084664 \mathrm{E}-40$

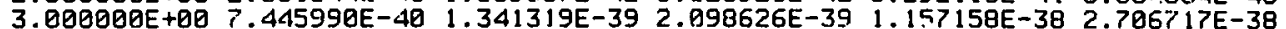

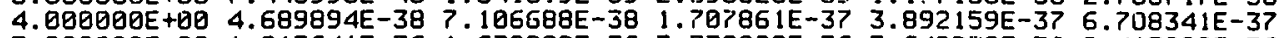

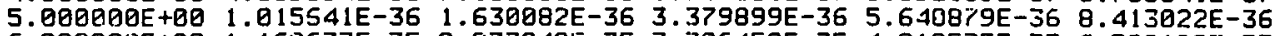
6. $000000 \mathrm{E}+00$ 1. $169633 \mathrm{E}-35$ 2.077940E-35 3.306452E-35 4.810535E-35 6.590192E-35 P. 000000E+00 $9.882314 \mathrm{E}-35 \quad 1.492165 \mathrm{E}-34 \quad 2.106848 \mathrm{E}-34 \quad 2.832279 \mathrm{E}-34 \quad 3.842055 \mathrm{E}-34$ $8.000000 \mathrm{E}+005.515183 \mathrm{E}-347.544622 \mathrm{E}-34 \quad 9.930430 \mathrm{E}-34 \quad 1.268202 \mathrm{E}-33 \quad 1.741265 \mathrm{E}-33$ $9.000000 \mathrm{E}+0 \mathrm{C}^{2} .311271 \mathrm{E}-33$ 2.978303E-33 $3.742358 \mathrm{E}-33 \quad 4.845466 \mathrm{E}-33$ 6.261716E-33

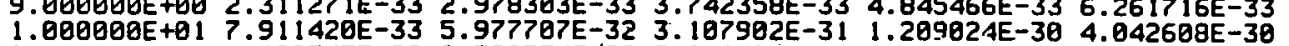
2.000000E+Q1 1.080743E-29 2.389554E-29 5.256394E-29 9.515209E-29 1.809750E-28 $3.000000 \mathrm{E}+01 \mathrm{1} 2.986464 \mathrm{E}-28$ 5. $260525 \mathrm{E}-28$ 7.864247E-28 $1.213125 \mathrm{E}-27 \quad 1.786571 \mathrm{E}-27$ 4. 0000ด0E+01 2.576148E-27 3.617182E-27 5.001597E-27 6.862255E-27 9.125677E-27

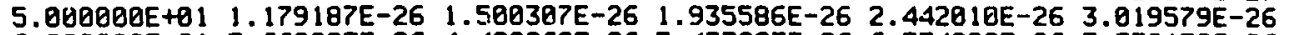
6.000000E+ 1 3.668293E-26 $4.499269 \mathrm{E}-26 \quad 5.457927 \mathrm{E}-26 \quad 6.534890 \mathrm{E}-26 \quad 7.730158 \mathrm{E}-26$ 7.000000E+Q1 9.135968E-26 $1.076983 E-25 \quad 1.258403 E-25 \quad 1.457858 E-25 \quad 1.680191 E-25$

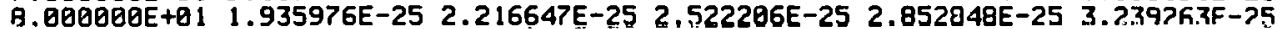

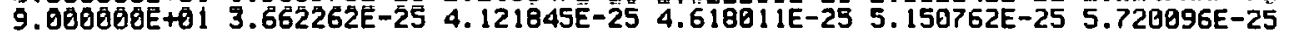
$1.000000 \mathrm{E}+02$ 6.326014E-25 $1.529914 \mathrm{E}-24$ 3.07 $1314 \mathrm{E}-24 \quad 5.445744 \mathrm{E}-24$ 8.855142E-24 2.000000E+02 $1.346057 \mathrm{E}-23$ 1.927054E-23 $2.644957 \mathrm{E}-23 \quad 3.495200 \mathrm{E}-23 \quad 4.599991 \mathrm{E}-23$

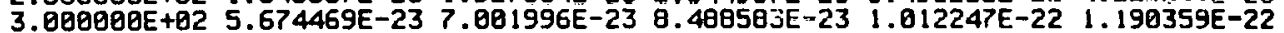
4. $000000 \mathrm{E}+02$ 1.383379E-22 $1.591307 \mathrm{E}-22 \quad 1.015526 \mathrm{E}-22 \quad 2.056873 \mathrm{E}-22 \quad 2.314270 \mathrm{E}-22$ 5. 6.000000E+02 $4.134456 \mathrm{E}-22$ 4.478597E-22 4.834280E-22 $5.201685 \mathrm{E}-22$ 5.580812E-22 7.000000E+02 5.970321E-22 6.369950E-22 6.790415E-22 7.201692E-22 7.633790E-22 8. 000000E+02 B. $076708 E-22$ 8.530447E-22 8.995007E-22 9.470387E-22 9.956508E-22 $9.000000 \mathrm{t}+22$ 1.045361E-21 $1.096145 \mathrm{E}-21 \quad 1.148011 \mathrm{E}-21 \quad 1.200960 \mathrm{E}-21 \quad 1.254990 \mathrm{E}-21$ $1.000000 \mathrm{E}+03$ 1.310102E-21

A19 SIGVJ $3 H E+7 L I \rightarrow P(3852)+A(1512)+A(1512)+N(3852) \cdot$ REF 5

$T(J)$ IN KEV SIGVK (J) IN M3/SEC

$1.000000 \mathrm{E}+00 \quad 5.773909 \mathrm{E}-41 \quad 1.186738 \mathrm{E}-39 \quad 1.512530 \mathrm{E}-38 \quad 1.215362 \mathrm{E}-37 \quad 9.275141 \mathrm{E}-37$ 2.000000E+00 $3.815080 \mathrm{E}-36 \quad 1.228418 \mathrm{E}-35 \quad 4.100156 \mathrm{E}-35 \quad 1.011411 \mathrm{E}-34 \quad 4.120404 \mathrm{E}-34$

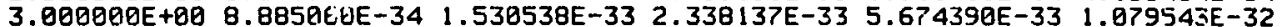

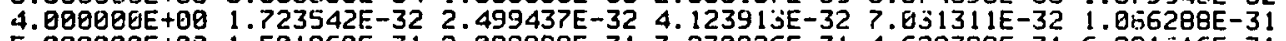
$5.900000 \mathrm{E}+00 \quad 1.501860 \mathrm{E}-31 \quad 2.992898 \mathrm{E}-31 \quad 3.230826 \mathrm{E}-31 \quad 4.628359 \mathrm{E}-31 \quad 6.29101 \mathrm{EE}-31$ 6. .00อ00E+0日 $8.220478 \mathrm{E}-31$ 1.153975E-30 $1.569695 \mathrm{E}-302.059: 17 \mathrm{E}-302.624043 \mathrm{E}-30$

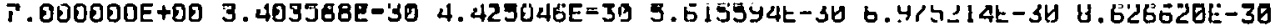
8. $000000 \mathrm{E}+00$ 1. $1.083758 \mathrm{E}-29$ 1.339120E-29 $1.629746 \mathrm{E}-29 \quad 1.953175 \mathrm{E}-292.300960 \mathrm{E}-29$ $9.000000 \mathrm{E}+00$ 2.869351E-29 $3.418246 \mathrm{E}-294.027646 \mathrm{E}-29$ 4.1 $40475 \mathrm{E}-29$ 5.640373E-29 $1.000000 \mathrm{E}+01$ 6.611664L-29 2.608190E-28 $7.030943 \mathrm{E}-28$ 1.935370E-27 4.191074E-27 $2.000000 \mathrm{E}+01$ 8. $134088 \mathrm{E}-2 \vec{r} \quad 1.408311 \mathrm{E}-26 \quad 2.355335 \mathrm{E}-26 \quad 3.611197 \mathrm{E}-26 \quad 5.448802 \mathrm{E}-26$

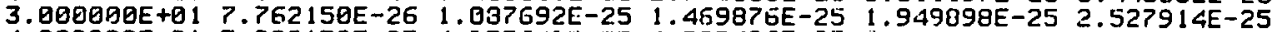

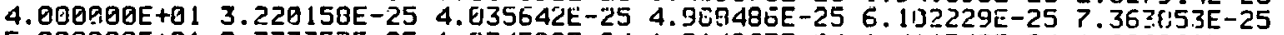

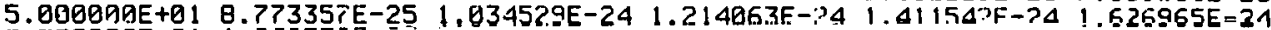

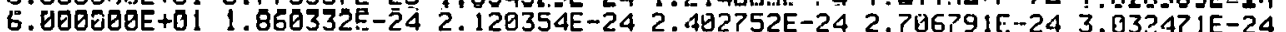
7.000000E+01 $3.385156 E-243.765261 E-24$ 4.171815E-24 4.603017E-24 5.06018 8.000000E+01 $5.544467 \mathrm{E}-24 \quad 6.054980 \mathrm{E}-24 \quad 6.591643 \mathrm{E}-24 \quad i .154-187 \mathrm{E}-247.740447 \mathrm{E}-24$ $9.000000 \mathrm{E}+01$ 1 $8.370437 \mathrm{E}-24 \quad 9.020458 \mathrm{E}-24 \quad 9.038509 \mathrm{E}-24 \quad 1.040459 \mathrm{E}-23 \quad 1.11387 \mathrm{BE}-23$ 1. Q000900E+02 $1.190084 E-23 \quad 2.110014 E-23 \quad 3.325052 E-23 \quad 4.829304 E-23 \quad 6.581301 E-23$

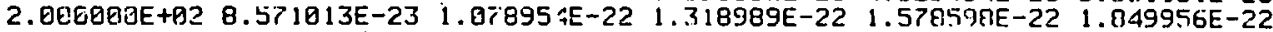

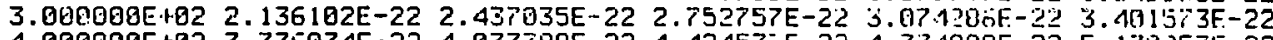

4.000000E+02 3.736074E-22 4.0777B9E-22 4.42453.JE-2. 4.7749A0E-22 5.133i353E-22

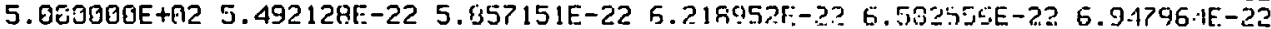

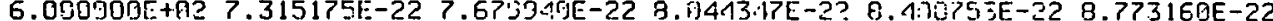

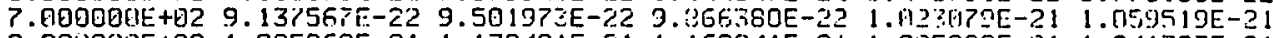

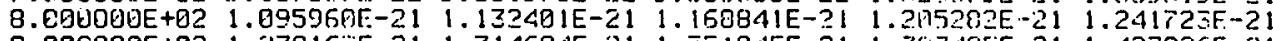

9. QRGG00E+02 $1.27816 \mathrm{GE}-21 \quad 1.314604 \mathrm{E}-21 \quad 1.551045 \mathrm{E}-21 \quad 1.38748 \mathrm{5E}-21 \quad 1.423926 \mathrm{E}-21$

1. $00000 \mathrm{UEE}+03$ 1.406367E-21

A20 SIGVK. $\quad$ I+7BE $\Rightarrow P(11179)+\cap(2795)+A(2795)$

REF : 
Table I (continued)

$T(J)$ IN KEV SIGVL ( $J$ ) IN MB/SEC

1.000000E+00 5. 186936E-43 $1.455643 E-412.501049 E-402.530489 E-392.615808 E-38$

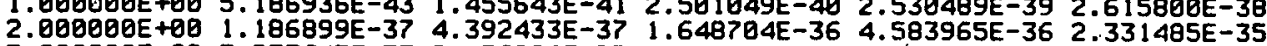
$3.000000 \mathrm{E}+00$ 5.237647E-35 9.17688.3E-35 1.414919E-34 4.055751E-34 8. $197925 \mathrm{E}-34$ 4. $000000 \mathrm{E}+0 \mathrm{\theta}$ 1.343924E-33 $1.977970 \mathrm{E}-33 \quad 3.515462 \mathrm{E}-33 \quad 6.437311 \mathrm{E}-33$ 1.012485E-32 5. $\theta 00000 \mathrm{E}+00$ 1. $457808 \mathrm{E}-32$ 2.099828E-32 $3.442313 \mathrm{E}-32 \quad 5.122037 \mathrm{E}-327.139903 \mathrm{E}-32$ 6.800000E+00 9.493208E-32 1.399057E-31 1.974403E-31 2.66011日E-31 3.456204E-31 7.800000E+00 4.628112E-31 $6.226225 \mathrm{E}-31$ 8.113247E-31 $1.028918 \mathrm{E}-301.301003 \mathrm{E}-30$ $8.000000 \mathrm{E}+08$ 1.683444E-30 $2.138988 \mathrm{E}-302.643636 \mathrm{E}-30 \quad 3.222433 \mathrm{E}-30 \quad 4.932062 \mathrm{E}-30$ $9.000000 \mathrm{E}+00$ 4.969554E-30 6.034908E-30 $7.228125 \mathrm{E}-30 \quad 8.733406 \mathrm{E}-30 \quad 1.053126 \mathrm{E}-29$ $\begin{array}{lllllll}1.000000 E+01 & 1.256078 E-29 & 5.774521 E-29 & 1.975915 E-28 & 5.495596 E-28 & 1.291705 E-27\end{array}$ $2.000000 \mathrm{E}+012.706817 \mathrm{E}-27$ 4.957502E-27 8.836871E-27 $1.413501 \mathrm{E}-262.248933 \mathrm{E}-26$

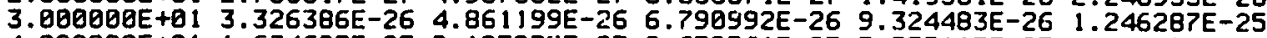
4. $00000 \theta \mathrm{E}+01$ 1.634623E-25 $2.10503 \mathrm{SE}-252.670761 \mathrm{E}-25 \quad 3.353113 \mathrm{E}-254.140303 \mathrm{E}-25$ $5.000000 \mathrm{t}+01$ 5.032332E-25 6.045297E-25 ?.246693E-25 $8.587255 E-25 \quad 1.006876 \mathrm{E}-24$ 6.000000E+01 $1.168948 \mathrm{E}-24 \quad 1.356041 \mathrm{E}-24 \quad 1.562733 \mathrm{E}-24 \quad 1.788090 \mathrm{E}-24 \quad 2.032112 \mathrm{E}-24$

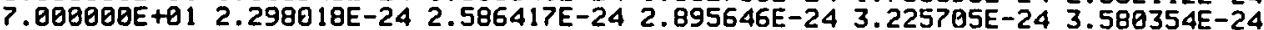
8.900000E+01 3.967801E-24 4.381397E-24 4.821143E-24 5.287093E-24 5.787696E-24 $9.000000 \mathrm{E}+016.317669 \mathrm{E}-24 \quad 6.877013 \mathrm{E}-24 \quad 7.465726 \mathrm{E}-24.8 .083910 \mathrm{E}-24 \quad 8.731263 \mathrm{E}-24$ $1.800000 \mathrm{t}+02$ 9.408087E-24 $1.794089 \mathrm{E}-23 \quad 2.991521 \mathrm{E}-23 \quad 4.552005 \mathrm{E}-23$ 6.459100E-23

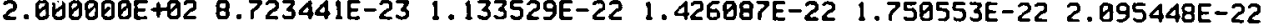
3. 000000E+02 $2.465229 \mathrm{E}-22$ 2.860432E-22 $3.280899 \mathrm{E}-22 \quad 3.719488 \mathrm{E}-22 \quad 4.176157 \mathrm{E}-22$ 4. $000090 \mathrm{E}+02$ 4.652063E-22 $5.147205 \mathrm{E}-225.654674 \mathrm{E}-22 \quad 6.170280 \mathrm{E}-22 \quad 6.699412 \mathrm{E}-22$ 5. $000000 \mathrm{E}+02$ 7.242070E-22 $7.796175 \mathrm{E}-22$ 8.353016E-22 $0.918074 \mathrm{E}-22$ 9.493748E-22 $6.000000 \mathrm{E}+021.007764 \mathrm{E}-21 \quad 1.065993 \mathrm{E}-21 \quad 1.124582 \mathrm{E}-21 \quad 1.183523 \mathrm{E}-21 \quad 1.243115 \mathrm{E}-21$ $\begin{array}{lllllll}7.000000 E+02 & 1.302789 E-21 & 1.362595 E-21 & 1.422672 E-21 & 1.483019 E-21 & 1.543636 E-21\end{array}$ $8.000009 \mathrm{E}+021.604524 \mathrm{E}-21 \quad 1.665683 \mathrm{E}-21 \quad 1.727112 \mathrm{E}-21 \quad 1.788812 \mathrm{E}-21 \quad 1.850782 \mathrm{E}-21$ $9.000000 E+02$ 1.913023E-21 $1.975534 E-212.038316 E-21 \quad 2.101360 E-212.164691 E-21$ $1.000000 \mathrm{E}+032.228284 \mathrm{E}-21$

$A 21$ SIGU $T+7 B E \rightarrow P(4204)+N(4204)+A(1051)+A(1851)$

REF 5

T(J) IN KEV SIGVM(J) IN M3/SEC

1.000000E+00 4.89437 1E-57 8.891207E-55 $1.161917 E-52 \quad 4.729856 E-51 \quad 4.212684 E-49$ 2. $000000 \mathrm{E}+00 \quad 2.515273 \mathrm{E}-4 \mathrm{\theta} \quad 2.903389 \mathrm{E}-47 \quad 1.723260 \mathrm{E}-46 \quad 1.912348 \mathrm{E}-45 \quad 2.433056 \mathrm{E}-44$ 3.000000E+00 6.001550E-44 1.009672E-43 1.711856E-43 1.972630E-42 4.701471E-42 3.

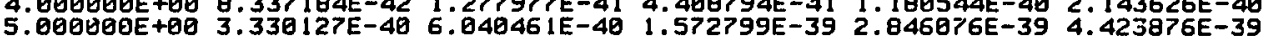
5.000009E+00 $3.330127 E-406.040461 E-40$ 1.572799E-39 $2.846076 E-39$ 4.423976E-39

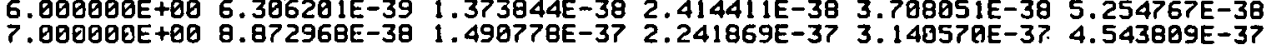
8.000000E+00 7.230415E-37 $1.056950 E-36 \quad 1.456107 \mathrm{E}-36 \quad 1.923377 \mathrm{E}-36 \quad 2.910075 \mathrm{E}-36$ $9.000000 E+00 \quad 4.134013 E-36 \quad 5.595191 E-36 \quad 7.293610 E-36 \quad 1.009613 E-35 \quad 1.390993 E-35$ $\begin{array}{lllllll}1.800090 E+Q 1 & 1.844962 E-35 & 2.153241 E-34 & 1.643181 E-33 & 0.737362 E-33 & 4.081835 E-32\end{array}$ 2. $.00000 E+\theta 1$ 1.348286E-31 3.4722.25E-31 $9.202734 \mathrm{E}-31$ 1.833977E-30 4.142537E-30

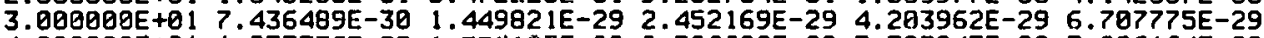
4. $000000 \mathrm{E}+\theta 1$ 1.053376E-28 $1.596123 \mathrm{E}-28 \quad 2.396688 \mathrm{E}-28 \quad 3.587845 \mathrm{E}-28$ 5.096164E-28 5.000000E+0 $16.921645 \mathrm{E}-28 \quad 9.243501 \mathrm{E}-28 \quad 1.281327 \mathrm{E}-27 \quad 1.708907 \mathrm{E}-27 \quad 2.207092 \mathrm{E}-27$

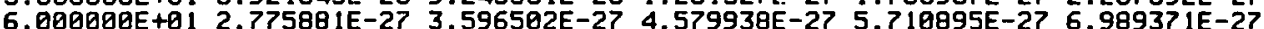
7. .000000E+01 B.592873E-27 $1.055509 E-26 \quad 1.278421 \mathrm{E}-26 \quad 1.520024 \mathrm{E}-26 \quad 1.816813 \mathrm{E}-26$ 8.000000E+01 $2.172051 \mathrm{E}-26 \quad 2.571653 \mathrm{E}-26.3 .015619 \mathrm{E}-26 \quad 3.504531 \mathrm{E}-26 \quad 4.129923 \mathrm{E}-26$ $9.000000 \mathrm{E}+01$ 4. $334563 \mathrm{E}-26 \quad 5.618450 \mathrm{E}-26 \quad 6.481586 \mathrm{E}-26 \quad 7.423968 \mathrm{E}-26 \quad 8.445599 \mathrm{E}-26$ $1.000000 \mathrm{E}+02 \quad 9.546477 \mathrm{E}-26 \quad 2.842141 \mathrm{E}-25 \quad 6.723794 \mathrm{E}-25 \quad 1.365985 \mathrm{E}-24 \quad 2.501160 \mathrm{E}-24$ $2.000000 \mathrm{E}+02 \quad 4.225873 \mathrm{E}-24 \quad 6.579142 \mathrm{E}-24 \quad 9.839941 \mathrm{E}-24 \quad 1.389701 \mathrm{E}-23 \quad 1.917392 \mathrm{E}-23$ $3.000000 \mathrm{E}+\mathrm{B2} 2 \mathrm{2}$ 2.550763E-23 $3.317533 \mathrm{E}-23 \quad 4.209671 \mathrm{E}-23 \quad 5.261170 \mathrm{E}-23 \quad 6.472224 \mathrm{E}-23$ 4. .00000E+Q2 $7.037170 E-23 \quad 9.35 E 009 E-23 \quad 1.194329 E-22 \quad 1.230783 E-221.493828 E-22$ 5. 6.000000E+02 3.102435E-22 3.43769PF-22 $3.791801 E-22 \quad 4.165000 E-22 \quad 4.557072 \mathrm{E}-23$

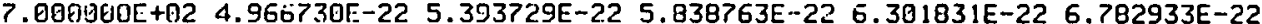
8.000000E+02 $7.282063 \mathrm{E}-22$ 7.799240E-22 $8.334445 \mathrm{E}-22$ 8.807C04E-22 $9.458957 \mathrm{E}-22$ $9.000060 \mathrm{E}+02$ 1.004826E-21 $1.065561 \mathrm{E}-21 \quad 1.128090 \mathrm{E}-21 \quad 1.192439 \mathrm{E}-21$ 1.258584E-21 $1.000000 \mathrm{E}+031.326532 \mathrm{E}-21$ 
Table I (continued)

T(J) IN KEV SIGWN(J) IN MB SEC

1. 2. $.00000 \mathrm{E}+00$ 1. $661651 \mathrm{E}-33$ 2.818156E-33 $7.726766 \mathrm{E}-33$ 1.639698E-32 4.804935E-32 3.000000E+80 $9.521830 E-32$ 1.579041E-31 $2.361065 \mathrm{E}-31 \quad 4.614269 \mathrm{E}-31$ ?.924391E-31 $4.000000 \mathrm{t}+60$ 1.203201E-30 $1.693711 \mathrm{E}-302.546318 \mathrm{E}-30 \quad 3.932107 \mathrm{E}-30 \quad 5.630978 \mathrm{E}-30$ $5.000000 \mathrm{E}+00$ 7.642924E-30 $1.022758 \mathrm{E}-291.447373 \mathrm{E}-291.959635 \mathrm{E}-292.559544 \mathrm{E}-29$ 6.000000E+0日 $3.247099 \mathrm{E}-29$ 4.270527E-29 5.527972E-29 $6.973010 \mathrm{E}-29$ 8.616อ42E-29

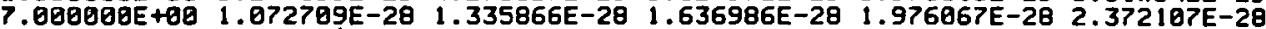

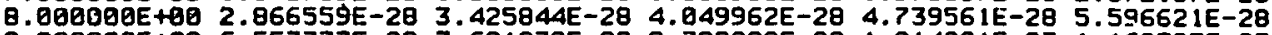
9.

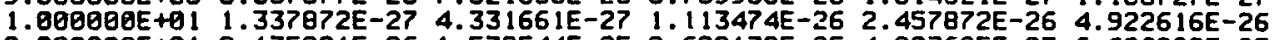
$2.000000 \mathrm{t}+0 \mathrm{i} 9.175201 \mathrm{E}-26 \quad 1.572544 \mathrm{E}-25 \quad 2.689178 \mathrm{E}-25 \quad 4.207625 \mathrm{E}-25 \quad 6.626209 \mathrm{E}-25$ $3.000000 \mathrm{E}+019.751375 \mathrm{E}-25 \quad 1.413800 \mathrm{E}-24 \quad 1.962534 \mathrm{E}-24 \quad 2.655824 \mathrm{E}-24 \quad 3.49 .3872 \mathrm{E}-24$ 4. $\theta 00000 E+81$ 4.479042E-24 5.614910E-24 6.890622E-24 8.297269E-24 9.839756E-24

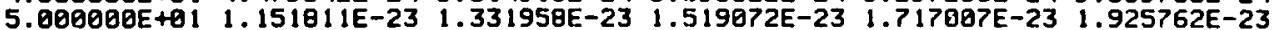
6.

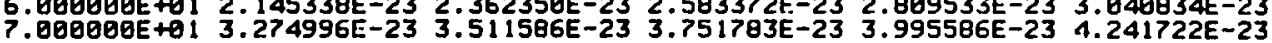
9.000000E+01 $4.407407 E-23 \quad 4.734996 E-23 \quad 4.984180 E-23 \quad 5.235267 E-23 \quad 5.485565 E-23$ $9.000008 E+815.736686 E-23 \quad 5.988633 E-23 \quad 6.241483 E-23 \quad 6.494999 E-23 \quad 6.749418 E-23$ $1.000000 \mathrm{E}+02$ 7.004662E-23 $9.527840 \mathrm{E}-23 \quad 1.191029 \mathrm{E}-22 \quad 1.40331 \mathrm{gE}-22 \quad 1.584423 \mathrm{E}-22$ $2.800000 \mathrm{E}+02$ 1.737428E-22 $1.865077 \mathrm{E}-22 \quad 1.979563 \mathrm{E}-22 \quad 2.075696 \mathrm{E}-22 \quad 2.153997 \mathrm{E}-22$

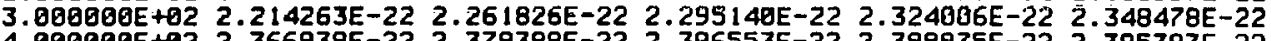
4. 5. $000000 \mathrm{E}+02$ 2.377289E-22 $2.365458 \mathrm{E}-22 \quad 2.359007 \mathrm{E}-22 \quad 2.351655 \mathrm{E}-22 \quad 2.343401 \mathrm{E}-22$ 6. 0日0อ日日E+02 2.334246E-22 $2.322064 E-22 \quad 2.307900 E-22 \quad 2.291932 E-22 \quad 2.27416 G E-22$ 7. $000000 \mathrm{E}+02$ 2.257267E-22 $2.241760 \mathrm{E}-22$ 2.226253E-22 $2.210747 \mathrm{E}-22 \quad 2.195240 \mathrm{E}-22$ $8.000000 \mathrm{E}+02$ 2.179733E-22 $2.164227 \mathrm{E}-222.148720 \mathrm{E}-22 \quad 2.133213 \mathrm{E}-22$ 2. $117707 \mathrm{E}-22$

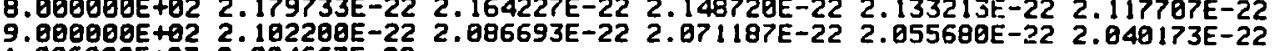
$1.006000 \mathrm{t}+032.024667 \mathrm{E}-22$

$T(J)$ IN KEV SIGVO(J) IN MB/SEC

1. $000000 E+00$ 1.091796E-37 $1.369555 E-36 \quad 1.112464 E-35 \quad 6.237082 E-35 \quad 3.104831 E-34$ $2.000000 \mathrm{E}+00 \quad 1.061651 \mathrm{E}-33 \quad 2.818156 \mathrm{E}-33 \quad 7.726766 \mathrm{E}-33 \quad 1.639698 \mathrm{E}-32 \quad 4.804935 \mathrm{E}-32$ $3.000000 \mathrm{E}+00 \quad 9.521838 \mathrm{E}-32$ 1.579041E-31 $2.361065 \mathrm{E}-31 \quad 4.614269 \mathrm{E}-31 \quad 7.924391 \mathrm{E}-31$

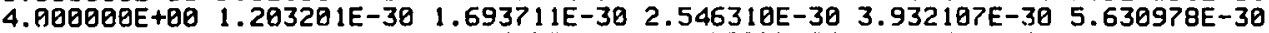
$5.000000 \mathrm{E}+007.642924 \mathrm{E}-301.022758 \mathrm{E}-291.447373 \mathrm{E}-291.959635 \mathrm{E}-292.559544 \mathrm{E}-29$ $6.000000 \mathrm{E}+90 \quad 3.247099 \mathrm{E}-29 \quad 4.278527 \mathrm{E}-29 \quad 5.527 \mathrm{JP} 2 \mathrm{E}-29 \quad 6.973810 \mathrm{E}-29$ 8.616042E-29 $\begin{array}{llllll}7.000000 E+00 & 1.072709 E-28 & 1.335866 E-28 & 1.636986 E-28 & 1.976057 E-28 & 2.372107 E-2 B\end{array}$

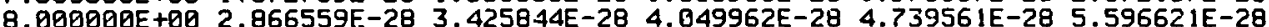
$9.00000 A \mathrm{E}+00$ 6.557377E-28 $7.621830 \mathrm{E}-28$ 8.709980E-28 $1.014821 \mathrm{E}-27$ 1. $160727 \mathrm{E}-27$

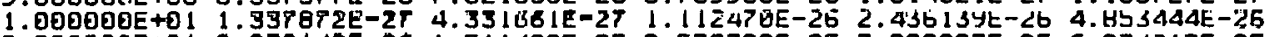

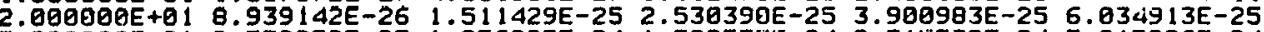
$3.000000 \mathrm{E}+\mathrm{I} \quad 0.772270 \mathrm{E}-251.256985 \mathrm{E}-241.729533 \mathrm{E}-24 \quad 2.315572 \mathrm{E}-24 \quad 3.015226 \mathrm{E}-24$ 4. . $5.000000 \mathrm{E}+01$ 9.706524E-24 $1.120811 \mathrm{E}-23 \quad 1.276805 \mathrm{E}-23 \quad 1.441815 \mathrm{E}-23 \quad 1.615842 \mathrm{E}-23$

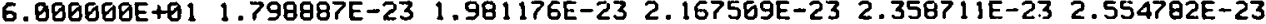

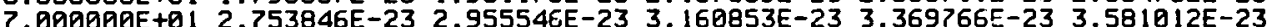

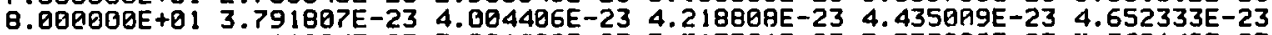
$9.009000 \mathrm{E}+01$ 4.87 1204E-23 5. $591 \mathrm{E} 2 \mathrm{EE}-23 \quad 5.313581 \mathrm{E}-23 \quad 5.537089 \mathrm{E}-23 \quad 5.762142 \mathrm{E}-23$ 1.000000E+02 $5.900741 \mathrm{E}-23$ 1.242107E-23 $1.036590 \mathrm{E}-22$ 1.224927E-22 $1.384609 \mathrm{E}-22$

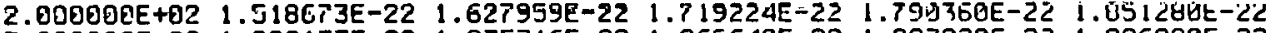

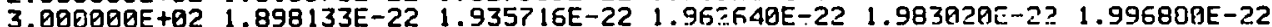

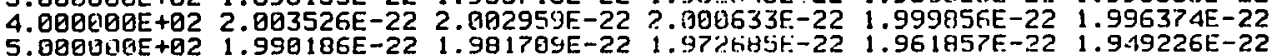

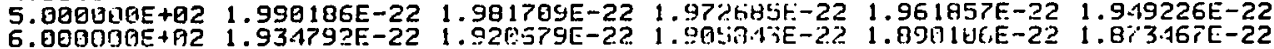

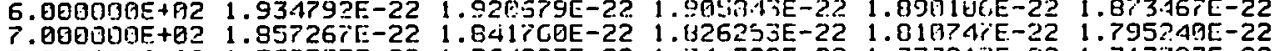

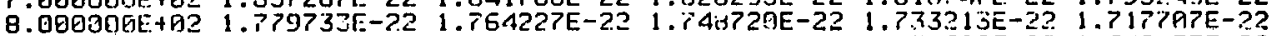

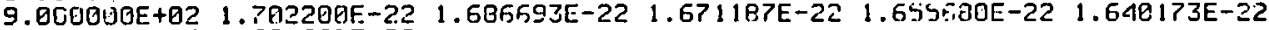
1. ตดอกดผ +ค.

A24 SIGVO $P+98 E \Rightarrow A(1275)+6 L I(85 \theta)$ 
Table I (continued)

T(J) IN KEV SIGVP(J) IN MB/SEC

$1.000000 E+00 \quad 6.626243 E-61 \quad 1.994537 E-58 \quad 4.074947 E-56 \quad 2.858616 E-54 \quad 4.721399 E-52$ 2. $3.020000 \mathrm{t}+08$ 8.605723E-46 $1.567662 \mathrm{E}-45 \cdot 2.466946 \mathrm{E}-45 \quad 7.203775 \mathrm{E}-441.807237 \mathrm{E}-43$ 4. $5.000000 \mathrm{t}+00 \quad 1.894707 \mathrm{E}-41 \quad 3.531799 \mathrm{E}-41 \quad 9.562143 \mathrm{E}-41 \quad 1.750390 \mathrm{E}-402.735707 \mathrm{E}-40$ 6. . 7.090000E+08 6.258567E-39 $1.089267 \mathrm{E}-38 \quad 1.669196 \mathrm{E}-38 \quad 2.365645 \mathrm{E}-38$ 3.497916E-38 8. $000000 \mathrm{E}+00$ 5.762741E-38 $8.595735 \mathrm{E}-38$ 1.199690E-37 $1.599107 \mathrm{E}-37$ 2.440920E-37 $9.000008 E+003.504593 E-37$ 4.766128E-37 6.233524E-37 9.583916E-37 1.174481E-36 1.000000E+0 1 1.549327E-36 $1.519485 E-35$ 1.053525E-34 8.473806E-34 1.007804E-32 2. 3.

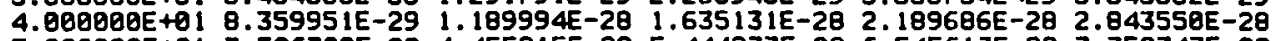
5. 000000E+01 3.596722E-28 4.455215E-28 5.444237E-28 $6.545613 \mathrm{E}-2 \mathrm{~B} \quad 7.759343 \mathrm{E}-28$ 6.000000E+01 $9.085426 \mathrm{E}-20 \quad 1.053109 \mathrm{E}-27 \quad 1.209277 \mathrm{E}-27 \quad 1.376988 \mathrm{E}-27 \quad 1.556241 \mathrm{E}-27$ $7.0000 \theta 0 E+01$ 1.754946E-27 $1.974603 \mathrm{E}-27 \quad 2.211123 \mathrm{E}-27 \quad 2.464585 \mathrm{E}-272.750750 \mathrm{E}-27$

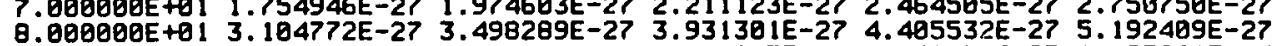
$9.000000 \mathrm{E}+016.122220 \mathrm{E}-27 \quad 7.194965 \mathrm{E}-27$ 7.410645E-27 $9.769259 \mathrm{E}-27$ 1.127001E-26 1. $00000 \mathrm{E}+02$ 1.291529E-26 $5.414255 \mathrm{E}-26$ 1.739711E-25 $4.497785 \mathrm{E}-25$ 9.314333E-25

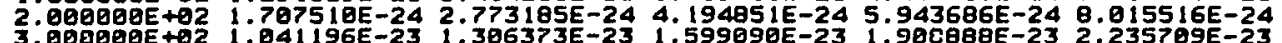

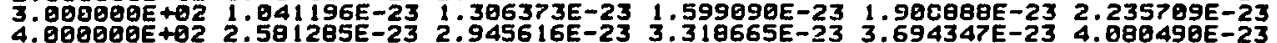
5. 6. $000000 \mathrm{E}+02$ 6.446121E-23 $6.835719 \mathrm{E}-23 \quad 7.224056 \mathrm{E}-23 \quad 7.611492 \mathrm{E}-23 \quad 7.998027 \mathrm{E}-23$ 7. $080000 \mathrm{E}+02$ 8.376956E-23 8.747098E-23 $9.111649 \mathrm{E}-23 \quad 9.470881 E-23$ 9.824702E-23 8.800000E+02 $1.017311 \mathrm{E}-221.051611 \mathrm{E}-22$ 1.885370E-22 $1.118508 \mathrm{E}-221.151265 \mathrm{E}-22$. $9.000000 \mathrm{E}+02$ 1. $183401 \mathrm{E}-22$ 1.214996E-2? $: 1.246050 \mathrm{E}-22 \quad 1.276563 \mathrm{E}-22 \quad 1.306535 \mathrm{E}-22$ 1. $.000000 \mathrm{E}+03$ 1.335966E-22

A25 ȘIGVP A+9BE $\Rightarrow N(5263)+12 C(439)$

REF 5

$T(J)$ IN KEV SIGVO(J) IN M3/SEC GLIGLI $\rightarrow$ P-11B

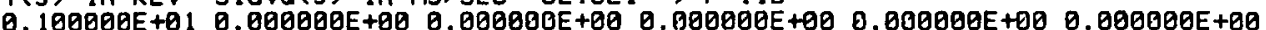

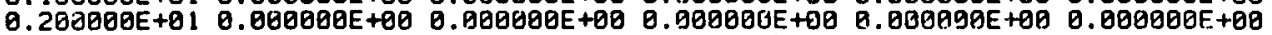
$0.300000 \mathrm{E}+01$ 0.000900E+00 0.900000E+00 0.000000E+00 0.000000E+00 0.000000E+00 $0.400000 \mathrm{E}+01$ 0.000000E+80 0.000000E+80 0.000000E+00 0.000000E-100 0.000000E+00

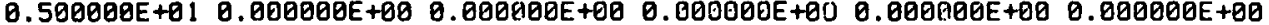

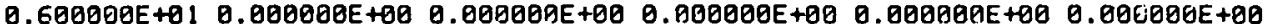

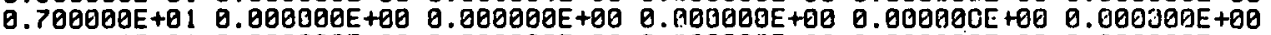
$0.800000 \mathrm{E}+01$ 0.000000E+00 .0.000000E +00 $0.0000000 \mathrm{E}+00$ 0.090000E+00 $0.000000 \mathrm{E}+00$ $0.900000 \mathrm{E}+01$ ด. $000000 \mathrm{E}+09$ 0.040000E+00 0.000000E+00 0.000000E+00 0.000000E+00 $0.100000 E+02 \quad 0.000000 E+00 \quad 0.000000 E+00 \quad 0.000000 E+00 \quad 0.000000 E+00 \quad 0.000000 E+00$

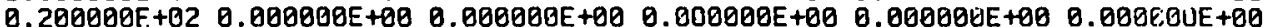

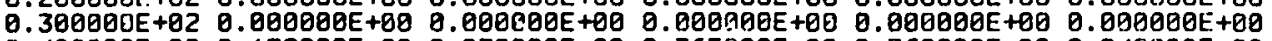

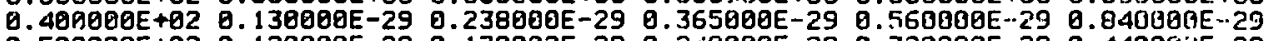
$0.500000 \mathrm{E}+02$ 0. $120000 \mathrm{E}-28$ 0.170030E-28 $0.240000 \mathrm{E}-28 \quad 0.320000 \mathrm{E}-20 \quad 0.44006 \mathrm{kE}-28$

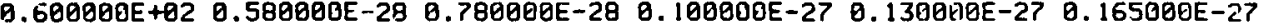
$\begin{array}{llllll}0.700000 E+02 & 0.208000 E-27 & 0.222764 E-27 & 0.254525 E-27 & 0.395 \mathrm{j} 197 \mathrm{E}-27 & 0.376297 E-27\end{array}$ $0.800000 \mathrm{E}+02$ 0.469939E-27 0.587838E-27 $0.731810 \mathrm{E}-27 \quad 0.903669 \mathrm{E}-27 \quad 0.118523 \mathrm{E}-26$ $0.900000 \mathrm{E}+02$ B. $133831 \mathrm{E}-26$ 0.160472E-26 $0.190628 \mathrm{E}-26$ 0.224490E-26 $0.262211 \mathrm{E}-2 \mathrm{E}$ $0.100000 \mathrm{t}+03$ 0.304000E-26 $0.102925 \mathrm{E}-25 \quad 0.262502 \mathrm{E}-25$ 0.553777E-25 $0.102140 \mathrm{E}-24$ $0.200000 \mathrm{E}+03 \quad 0.171000 \mathrm{E}-24 \quad 0.265711 \mathrm{E}-24 \quad 0.387176 \mathrm{E}-24 \quad 0.535585 \mathrm{E}-24$ 0.711130E-24

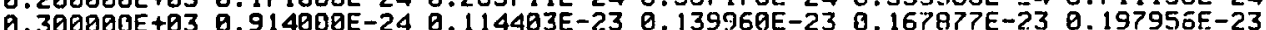
$0.400000 \mathrm{E}+03$ 0.230000E-23 $0.263815 \mathrm{E}-23$ Q. $29921 \mathrm{IE}-23 \quad 0.335909 \mathrm{E}-23 \quad 0.373992 \mathrm{E}-23$ $0.500000 \mathrm{E}+03 \quad 0.413000 \mathrm{E}-23 \quad 0.452354 \mathrm{~F}-23 \quad \mathrm{~g} .49345 \mathrm{BE}-23 \quad 0.534735 \mathrm{E}-23 \quad 0.5766 \mathrm{tgE}-23$

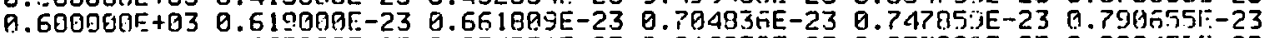

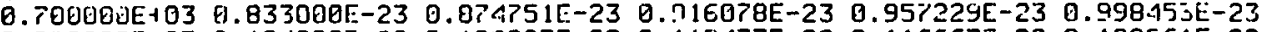

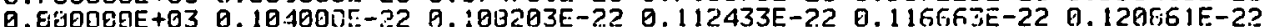

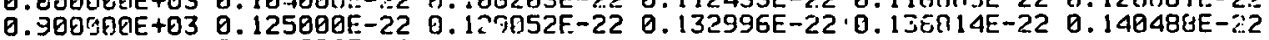

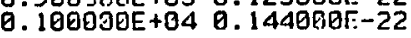


Table I (continued)

$T(J)$ IN KEV SIGVR(J) IN M3/SEC GLIELI $\rightarrow$ ALPHA-N-7BE

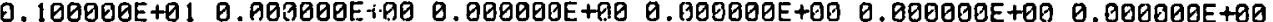

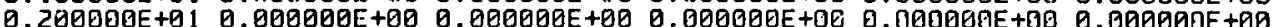

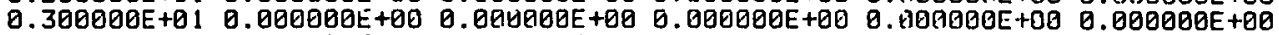
$0.400000 \mathrm{E}+0 \mathrm{I}$ 0. $000000 \mathrm{E}+00$ 0.000000E+00 0.000000E+00 0.000000E+00 0.000000E+00 $0.500000 E+01 \quad 0.000000 E+00 \quad 0.000000 E+00 \quad 0.000000 E+00 \quad 0.0<0000 E \cdot+00 \quad 0.000000 E+00$

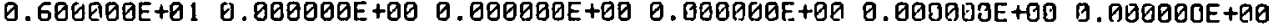

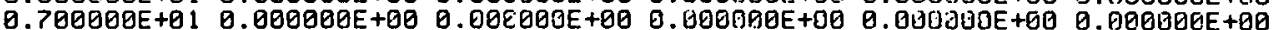

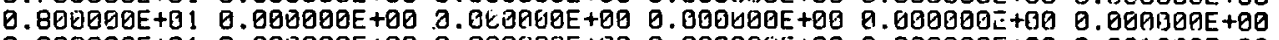

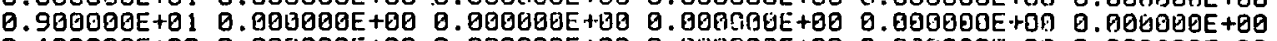

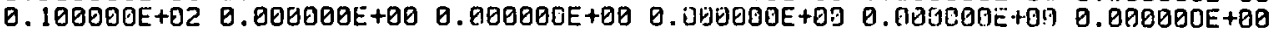

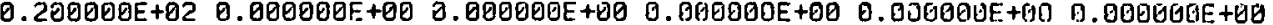

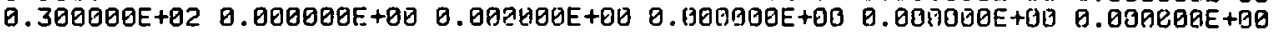
$0.400000 \mathrm{E}+02$ 0.820090E-29 $0.146000 \mathrm{E}-22$ 0.240000E-28 $0.370000 \mathrm{E}-28$ 0.540000E-28 $0.500000 \mathrm{E}+02$ 0.770000E-28 0.110000E-27 0.152000E-27 0.206000E-27 0.280000E-27 $0.600000 \mathrm{t}+02$ 0.370000E-27 0.490000E-27 0.630000E-27 0.810000E-27 0.104000E-26 $0.700000 \mathrm{E}+02 \quad 0.130000 \mathrm{E}-26 \quad 0.141966 \mathrm{E}-26 \quad 0.163752 \mathrm{E}-26 \quad 0.196412 \mathrm{E}-26 \quad 0.241000 \mathrm{E}-26$ $0.800000 \mathrm{E}+02$ 0.298569E-26 $0.370173 \mathrm{E}-26 \quad 0.456865 \mathrm{E}-26 \quad 0.559698 \mathrm{C}-26 \quad 0.679727 \mathrm{E}-26$ 0.980000E+02 $0.818005 \mathrm{E}-26 \quad 0.975585 \mathrm{E}-26 \quad 0.115352 \mathrm{E}-25 \quad 0.135297 \mathrm{E}-25 \quad 0.157468 \mathrm{E}-25$ 0.100000E+03 $0.182000 \mathrm{E}-25$ 0.68284IE-25 $0.151272 \mathrm{E}-24 \quad 0.315498 \mathrm{E}-24 \quad 0.577296 \mathrm{E}-24$ $0.200000 \mathrm{E}+03$ 0.961000E-24 0.143719E-23 0.216139E-23 $0.298541 \mathrm{E}-23$ 0.3961日2E-23

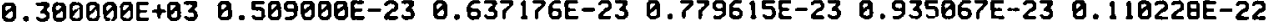
$0.49000 B E+03$ 0.12800日E-22 $0.146712 E-22$ 0.16630BE-22 $0.186749 E-22$ 0.207993E-22 0.500000E+03 $0.230000 \mathrm{E}-22$ 0.252720E-22 $0.276071 \mathrm{E}-22$ 0.299962E-22 $0.324392 \mathrm{E}-22$ $0.600000 \mathrm{E}+03$ 0.349000E-22 $0.373976 \mathrm{E}-22 \quad 0.399192 \mathrm{E}-22 \quad 0.424619 \mathrm{E}-22 \quad 0.450231 \mathrm{E}-22$ $0.700000 \mathrm{E}+03$ 0.476000E-22 $0.511896 \mathrm{E}-22$ 0.527802E-22 $0.553921 \mathrm{E}-22$ 0.579973E-22 $0.000000 E+03$ 0.6960日日E-22 $0.631968 E-22 \quad 0.657863 E-22 \quad 0.683673 E-22 \quad 0.709389 E-22$ $0.100000 \mathrm{E}+04 \mathrm{0.061000 \textrm {E } - 2 2}$

A27 SIGVR 6LI+6LI $\Rightarrow A(342)+N(137 \theta)+7 B E(196)$

REF $?$

$T(\mathrm{~J})$ IN KEV SIGVS(J) IN M3/SEC 6LI6LI $\rightarrow$ 3-ALPHA

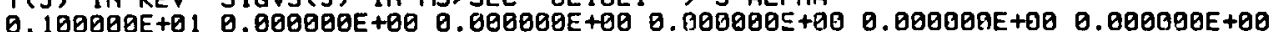

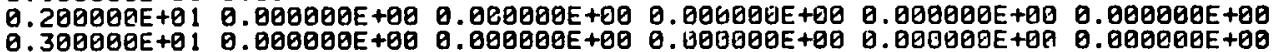

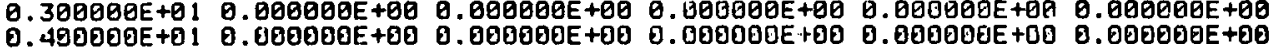
$0.500000 E+01$ O. $000000 E+00$ B. $000000 E+00$ 0.000000E+00 0.000000F+00 0.000006E+00

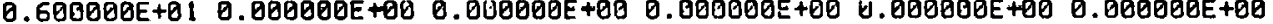
$0.700000 E+01$ 0.000000E+00 0.000000E+00 0.000000E+00 B.000000E+00 0.000000E+00 B.

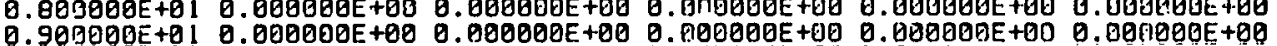

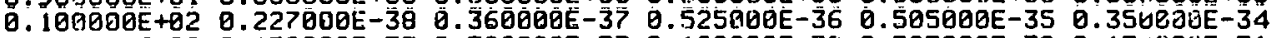

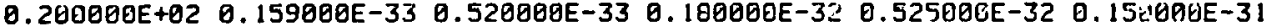

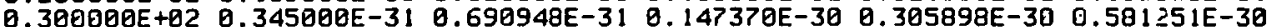
$0.400000 \mathrm{E}+02 \quad 0.101000 \mathrm{E}-29 \quad 0.166364 \mathrm{E}-29 \quad 0.275335 \mathrm{E}-29 \quad 0.452525 \mathrm{E}-29 \quad 0.722543 \mathrm{E}-29$ 0.580000E+02 $0.111006 E-28 \quad 0.164894 \mathrm{E}-28 \quad 0.241118 \mathrm{E}-28 \quad 0.347794 \mathrm{E}-28 \quad 0.493047 \mathrm{E}-28$ $0.600000 \mathrm{E}+02$ 0.685900E-28 $0.934021 \mathrm{E}-28$ 0.125945E-27 $0.168287 \mathrm{E}-27$ 0.222586E-27 0.709000E+Q2 $0.291000 \mathrm{E}-27 \quad 0.375909 E-27 \quad 0.480578 E-27 \quad 0.608493 E-27 \quad 0.76313 d E-27$

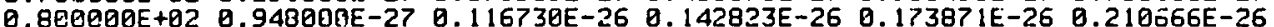
$0.900000 \mathrm{E}+02 \quad 0.254000 \mathrm{E}-26 \quad 0.304606 \mathrm{E}-26 \quad 0.362976 E-26 \quad 0.429543 \mathrm{E}-26 \quad 0.504740 \mathrm{E}-26$ $0.100000 \mathrm{E}+03 \quad 0.589000 \mathrm{E}-26 \quad 0.222066 \mathrm{E}-25 \quad 0.6407$ IOE-25 $0.147612 \mathrm{E}-24 \quad 0.290710 \mathrm{E}-24$ $0.200000 \mathrm{E}+03 \quad 0.513000 \mathrm{E}-24 \quad 0.032621 \mathrm{E}-24 \quad 0.126143 \mathrm{E}-23 \quad 0.100960 \mathrm{E}-23 \quad 0.248435 \mathrm{E}-23$

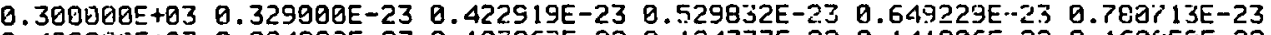

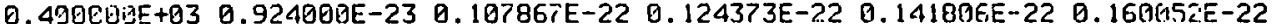
$0.500000 \mathrm{E}+03$ 0.179000E-22 $0.198549 \mathrm{E}-22 \quad 0.218656 \mathrm{E}-22 \quad 0.234287 \mathrm{E}-22 \quad 0.2601412 \mathrm{E}-22$

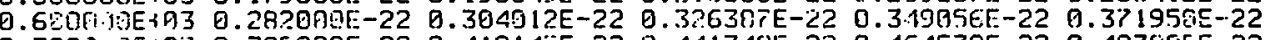

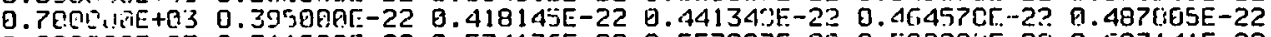

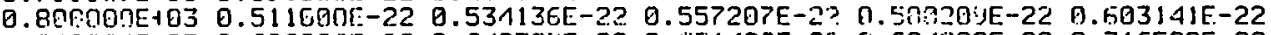

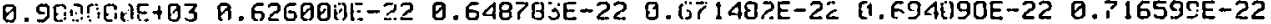

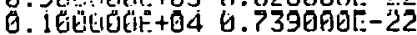

A23 SIGVS 6LI+6LI $\Rightarrow A(6967)+A(6967)+A(6967)$ 
Table I (continued)

$T$ (J) IN KEV SICIVT(J) IN MB/SEC GLI6LI $\rightarrow \mathrm{N}-11 \mathrm{C}$

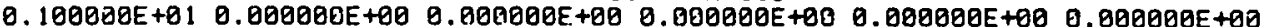

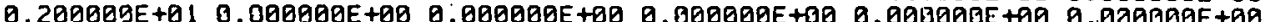

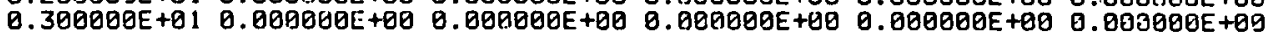
$0.400000 \mathrm{E}+01$ 0.000000E+00 $0.000000 \mathrm{E}+00$ 0.000000E+00 $0.000000 \mathrm{E}+00$ 0.000ดด0E+00

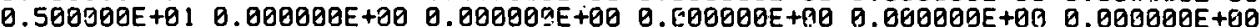

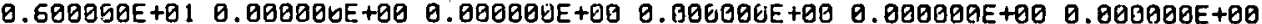

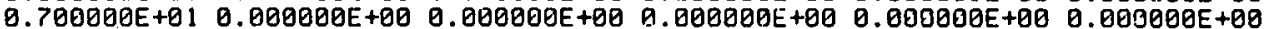

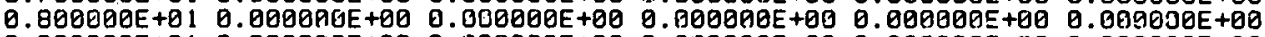
$0.900000 \mathrm{E}+01$ 0.080000E+00 0. $000 \mathrm{CO0E}+00$ 0.000000E+00 0.000000E+00 0.000000E+0日

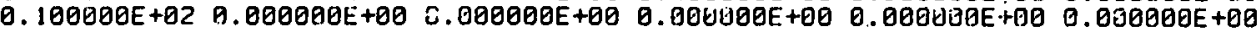

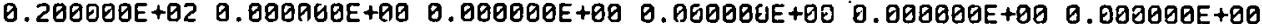

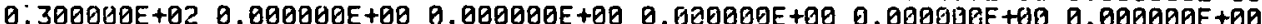
$0.400000 \mathrm{E}+02$ 0.126000E-29 0.210000E-29 $0.370000 \mathrm{E}-29 \quad 0.505000 \mathrm{E}-29 \quad 0.75060 \mathrm{EE}-29$ $0.500900 \mathrm{E}+02$ 0.110000E-28 $0.155000 \mathrm{E}-28 \quad 0.218000 \mathrm{E}-28 \quad 0.300000 \mathrm{E}-28 \quad 0.405000 \mathrm{E}-28$ $0.600000 \mathrm{E}+02$ 0.550000E-28 $0.720000 \mathrm{E}-28 \quad 0.950000 \mathrm{E}-28 \quad 0.122000 \mathrm{E}-27 \quad 0.155000 \mathrm{E}-27$ $\begin{array}{lllllll}0.7013 E E 0 E+02 & 0.19900 G E-27 & 0.200724 E-27 & 0.217183 E-27 & 0.250856 E-27 & 0.304222 E-27\end{array}$ $0.800000 \mathrm{t}+2 \mathrm{2}$ 0.379760E-27 0.479950E-27 0.607269E-27 0.764198E-27 0.953216E-27 $0.900000 \mathrm{E}+02$ $0.117680 \mathrm{E}-26 \quad 0.143743 \mathrm{E}-26 \quad 0.173759 \mathrm{E}-26 \quad 0.207975 \mathrm{E}-26 \quad 0.246639 \mathrm{E}-26$ $0.100000 E+03$ 0.290000E-26 0.105064E-25 $0.263803 E-25 \quad 0.538770 E-25 \quad 0.963038 E-25$ $0.200000 \mathrm{t}+03 \quad 0.157000 \mathrm{E}-24 \quad 0.238328 E-24 \quad 0.338774 E-24 \quad 0.455857 E-24 \quad 0.587093 E-24$

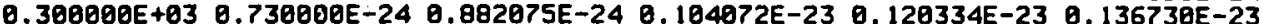
$0.400000 \mathrm{E}+03$ 0.153000E-23 $0.168920 \mathrm{E}-23$ 0.184416E-23 $0.199452 \mathrm{E}-23$ 0.213992E-23

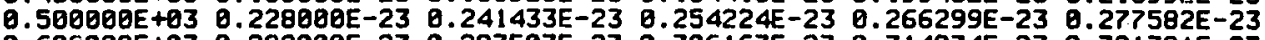
$0.600000 \mathrm{t}+03$ Q.288000E-23 $0.297507 \mathrm{E}-23 \quad 0.306167 \mathrm{E}=23 \quad 0.314074 \mathrm{E}=23 \quad 0.321321 \mathrm{E}-23$ $0.700000 \mathrm{t}+03 \quad 0.328000 \mathrm{E}-23 \quad 0.33418 \mathrm{EE}-23$ 0.339892E-23. $0.345102 \mathrm{E}-23 \quad 0.349808 \mathrm{E}-23$ $0.808080 E+03$ B.354080E-23 $0.357677 \mathrm{E}-23 \quad 0.360874 \mathrm{E}-23 \quad 0.363631 \mathrm{E}-23 \quad 0.365992 \mathrm{E}-23$

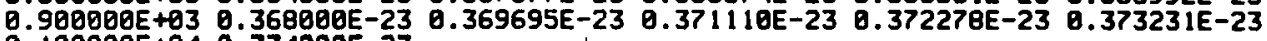
$0: 100000 \mathrm{E}+040.374000 \mathrm{E}-23$

A29 S.IGVT 6LI+6LI $\Rightarrow N(8665)+11 C(788)$

$T(J)$ IN KEV SIGVU(J) IN M3/SEC 6LIGLI $\rightarrow$ D-10B

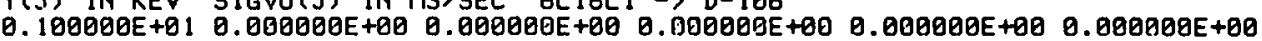
$0.200200 E+01$ 0.000000E+00 $0.000000 E+00$ 0.000000E+00 $0.000000 E+00$ 0.000000E+00

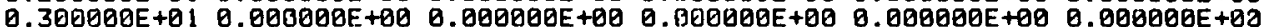

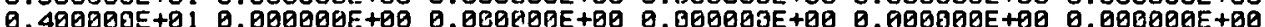

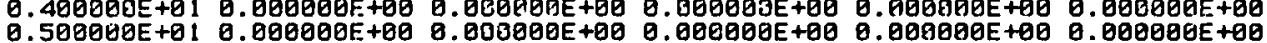

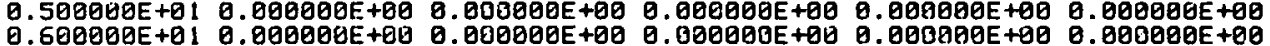

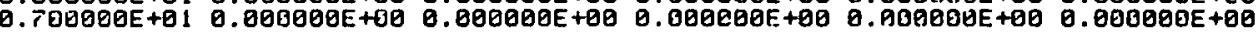

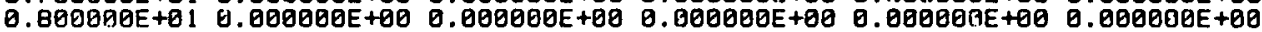

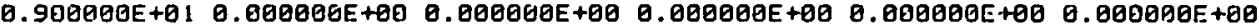

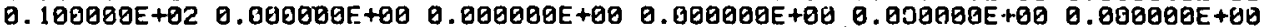

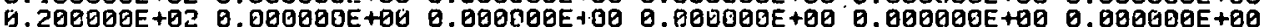

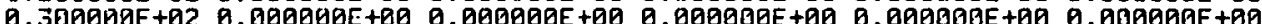

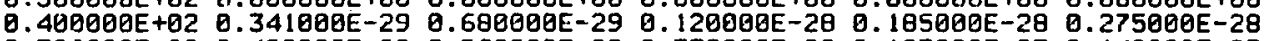

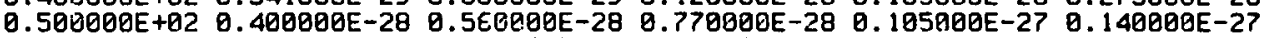
$\begin{array}{llllll}0.600009 E+02 & 0.190000 E-27 & 0.245000 E-27 & 0.318000 E-27 & 0.405001 E-27 & 0.525000 E-27\end{array}$ $0.700009 E+02$ 0.548000E-27 0.690710E-27 $0.698994 E-27 \quad 0.846903 E-27 \quad 0.104851 E-25$ $0.801000 E+02 \quad 0.130786 E-26 \quad 0.162901 E-26 \quad 0.201603 E-26 \quad 0.247296 E-26 \quad 0.300386 E-26$

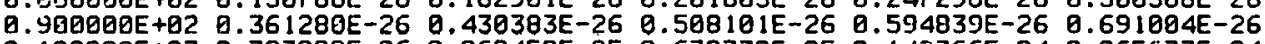
0.100000E+e3 $0.797000 \mathrm{E}-26 \quad 0.262459 \mathrm{E}-25 \quad 0.670732 \mathrm{E}-25 \quad 0.142766 \mathrm{E}-24 \quad 0.265637 \mathrm{E}-24$ $0.200000 \mathrm{E}+0.3 \quad 0.448000 \mathrm{E}-24 \quad 0.700624 \mathrm{E}-24 \quad 0.102810 \mathrm{E}-23 \quad 0.143345 \mathrm{E}-23 \quad 0.191974 \mathrm{E}-23$ $0.300000 \mathrm{E}+03$ 0.249000E-23 $0.314625 \mathrm{E}-23$ 0.388644E-23 $0.470751 \mathrm{E}-23$ 0.560639E-23 0.4 U日U00E+03 $0.650000 \mathrm{E}-23$ 0.762479E-23 $0.073522 \mathrm{E}-23$ 0.990525E-23 $0.111289 \mathrm{E}-22$ $0.500009 \mathrm{E}+\mathrm{E} 3 \mathrm{3}$ 0.124000E-22 0.137130E-22 $0.150635 \mathrm{E}-22$ 0.16447-iE-22 $0.178610 \mathrm{E}-22$

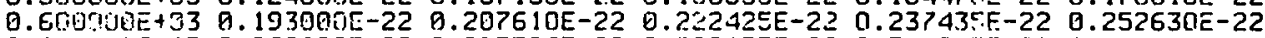

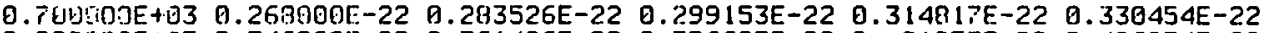
0. B00000E+1)3 $0.346000 E-22 \quad 0.361406 E-22 \quad 0.376683 E-22 \quad 0.391857 E-22 \quad 0.406954 E-22$ $0.500000 \mathrm{E}+03$ 0.422006E-22 0.437013E-22 0.452019E-22 0.467011E-22 0.482002E-22

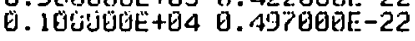


Table I (continued)

T(J) IN KEV SIGWV(J) IN MB/SEC GLIGLI $\rightarrow$ T-9B

$0.100000 \mathrm{E}+01$ 0.090000E+00 0.000000E+00 0.000000E+00 0.000000E+00 9.000000E+09 $0.200900 E+81$ 0.000000E+20 0.000000E+00 0.000000E+00 0.000000E+ $0.300000 \mathrm{E}+01$ 0.000000E+00 0.000000E+00 0.000000E+00 0.000100E+00 0.000001E+00 $0.400000 \mathrm{E}+01$ 0.000000E+00 0.000000E+00 $0.000000 \mathrm{E}+00$ 0.000000E+00 $0.000000 \mathrm{E}+00$ $0.500000 \mathrm{C}+01$ Q.000000E+00 0.000000E+00 0.900000E+00 0.000000E+00 0.000000E+00

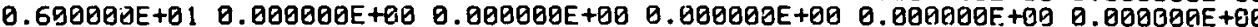

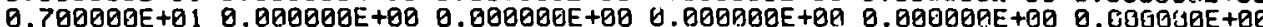

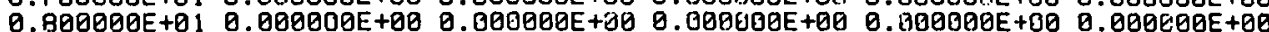

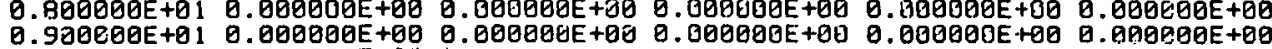
0. $100000 \mathrm{E}+02$ 0.000000E+00 0.000006E+00 0.000000E+00 0.000000E+00 0.000000E+00

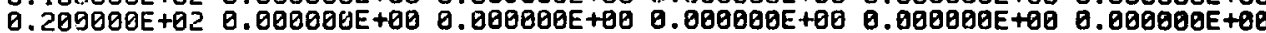

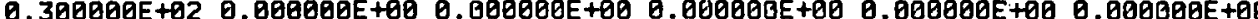

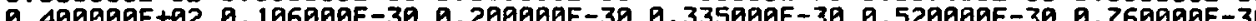
$0.400000 \mathrm{E}+02 \quad 0.106000 \mathrm{E}-30 \quad 0.200000 \mathrm{E}-30 \quad 0.335000 \mathrm{E}-30 \quad 0.520000 \mathrm{E}-30 \quad 0.760000 \mathrm{E}-30$ $0.500000 \mathrm{E}+02$ $0.110000 \mathrm{E}-29 \quad 0.154000 \mathrm{E}-29 \quad 0.210000 \mathrm{E}-29 \quad 0.205000 \mathrm{E}-29 \quad 0.360000 \mathrm{E}-29$ $0.600000 \mathrm{E}+02$ 0.500000E-29 $0.650000 \mathrm{E}-290.840000 \mathrm{E}-29$ 0.109000E-28 $0.138000 \mathrm{E}-2 \mathrm{~B}$ 0.700000E+02 0.171000E-28 0.183822E-28 0.218619E-28 0.252861E-28 0.312019E-28

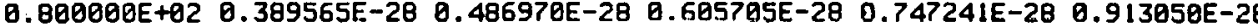
$0.900000 \mathrm{E}+02$ 0.110460E-27 0.132337E-27 0.157082E-27 0.184843E-27 0.215766E-27

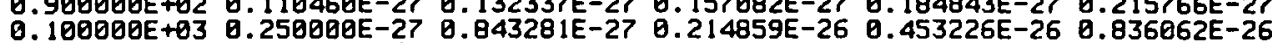
$0.200000 \mathrm{E}+03$ 0.140000E-25 $0.217569 \mathrm{E}-25$ 0.316902E-25 $0.430311 \mathrm{E}-25$ 0.581626E-25 $0.300003 \mathrm{E}+03$ 0.747000E-25 0.934285E-25 0.114246E-24 0.137030E-24 0.161656E-24 $0.400000 \mathrm{E}+03$ 0.188000E-24 $0.215930 \mathrm{E}-24 \quad 0.245275 \mathrm{E}-24 \quad 0.275855 \mathrm{E}-24 \quad 0.307490 \mathrm{E}-24$ 0.5000日0E+03 0.340090E-24 0.373226E-24 日. 407093E-24 $0.441517 \mathrm{~F}-24$ 0.476534E-24

$0.600000 \mathrm{E}+93$ 0.512000E-24 $0.547986 \mathrm{E}-24$ 0.5B4112E-24 $0.62059 \mathrm{GE}-24 \quad 0.657253 \mathrm{E}-24$ $0.700000 E+03$ 0.694000E-24 0.739751E-24 0.757410E-24 0.803908E-24 0.840133E-24 B.899000E+03 0.87600GE-24 0.911445E-24 0.946505E-24 0.981242E-24 0.101572E-23

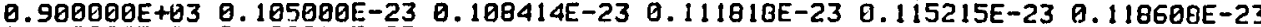

$0.106000 \mathrm{E}+04$ 0.122000E-23

A3I SIGW 6LI+6LI $\Rightarrow T(606)+9 B(202)$ 
Table II. Summary of reactions, <sigma v>, tabular references, and selected energy releases in $\mathrm{keV}$ (usually inversely proportional to mass)

\begin{tabular}{|c|c|c|c|}
\hline $\mathrm{A} 01$ & SIGV1 & $\mathrm{D}+\mathrm{D} \rightarrow 3 \mathrm{HE}(817)+\mathrm{N}(2450)$ & \\
\hline $\mathrm{A} 02$ & SIGV2 & $\mathrm{D}+\mathrm{D} \rightarrow \mathrm{T}(1008)+\mathrm{P}(3024)$ & \\
\hline $\mathrm{A} 03$ & SIGV3 & $\mathrm{D}+\mathrm{T} \rightarrow \mathrm{A}(3517)+\mathrm{N}(14069)$ & \\
\hline $\mathrm{A} 04$ & SIGV4 & $\mathrm{T}+\mathrm{T} \rightarrow \mathrm{A}(1259)+\mathrm{N}(5034)+\mathrm{N}(5034)$ & \\
\hline \multirow[t]{3}{*}{ A05 } & SIGV5 & $\mathrm{T}+3 \mathrm{HE} \rightarrow \mathrm{D}(9546)+\mathrm{A}(4773)$ & $41 \%$ of SIGV5 \\
\hline & & $P(5374)+A(1344)+N(5374)$ & $55 \%$ of SIGV5 \\
\hline & & $P(10077)+A(403)+N(1612)$ & $4 \%$ of SIGV5 \\
\hline
\end{tabular}

REF 1

A06 SIGV6 $\quad \mathrm{D}+3 \mathrm{HE} \rightarrow \mathrm{P}(14681)+\mathrm{A}(3670)$

REF 1

A07 SIGV7 D+6LI $\rightarrow 7 B E(423)+N(2957)$

REF 1

A08 SIGV8 D+6LI $\rightarrow 7 \mathrm{LI}(628)+\mathrm{P}(4398)$

REF 1

A09 SIGV9

$\mathrm{D}+6 \mathrm{LI} \rightarrow \mathrm{P}(1618)+\mathrm{A}(404)+\mathrm{T}(539)$

REF 2

A10 SIGVA

$D+6 L I \rightarrow A(11187)+A(11187)$

REF 2

REF 2

REF 1

REF 3

REF 3

A11 SIGVB

$\mathrm{D}+6 \mathrm{LT} \rightarrow 3 \mathrm{HE}(378)+\mathrm{A}(284)+\mathrm{N}(1134)$

REF 3

A12 SIGVC

$3 \mathrm{HE}+6 \mathrm{LI} \rightarrow \mathrm{P}(12390)+\mathrm{A}(2245)+\mathrm{A}(2245)$

REF 3

A13 SIGVD

$$
\mathrm{P}+6 \mathrm{LI} \rightarrow 3 \mathrm{HE}(2298)+\mathrm{A}(1724)
$$

A14 SIGVE

$3 \mathrm{HE}+3 \mathrm{HE} \rightarrow \mathrm{P}(5716)+\mathrm{P}(5716)+\mathrm{A}(1429)$

A15 SIGVF

$\mathrm{P}+11 \mathrm{~B} \rightarrow \mathrm{A}(2888)+\mathrm{A}(2888)+\mathrm{A}(2888)$

REF 3

A16 SIGVG

$\mathrm{P}+7 \mathrm{LI} \rightarrow \mathrm{A}(8674)+\mathrm{A}(8674)$

A17 SIGVH

$\mathrm{D}+7 \mathrm{LI} \rightarrow \mathrm{N}(10082)+\mathrm{A}(2521)+\mathrm{A}(2521)$

A18 SIGVI

$\mathrm{T}+7 \mathrm{LI} \rightarrow \mathrm{N}(6049)+\mathrm{N}(6049)+\mathrm{A}(1512)+\mathrm{A}(1512)$

A19 SIGVJ

$3 \mathrm{HE}+7 \mathrm{LI} \rightarrow \mathrm{P}(3852)+\mathrm{A}(1512)+\mathrm{A}(1512)+\mathrm{N}(3852)$

REF 4

REF 4

REF 5

A20 SIGVK

$\mathrm{D}+7 \mathrm{BE} \rightarrow \mathrm{P}(11179)+\mathrm{A}(2795)+\mathrm{A}(2795)$

REF 6

REF 5

REF 5

REF 5

REF 5

A21 SIGVL

$\mathrm{T}+7 \mathrm{BE} \rightarrow \mathrm{P}(4204)+\mathrm{N}(4204)+\mathrm{A}(1051)+\mathrm{A}(1051)$

REF 5

REF 5

A22 SIGVM

$3 \mathrm{HE}+7 \mathrm{BE} \rightarrow \mathrm{P}(45.10)+\mathrm{P}(4510)+\mathrm{A}(1127)+\mathrm{A}(1127)$

REF 5

A23 SIGVN

$\mathrm{P}+9 \mathrm{BE} \rightarrow \mathrm{D}(326)+\mathrm{A}(163)+\mathrm{A}(163)$

REF 5

A24 SIGVO

$\mathrm{P}+9 \mathrm{BE} \rightarrow \mathrm{A}(1275)+6 \mathrm{LI}(850)$

REF 5

A25 SIGVP

$\mathrm{A}+9 \mathrm{BE} \rightarrow \mathrm{N}(5263)+12 \mathrm{C}(439)$

REF 5

A26 SIGVQ

$6 \mathrm{LI}+6 \mathrm{LI} \rightarrow \mathrm{P}(11200)+11 \mathrm{~B}(10.18)$

REF 7

A27 SIGVR

$6 \mathrm{LI}+6 \mathrm{LI} \rightarrow \mathrm{A}(342)+\mathrm{N}(1370)+7 \mathrm{BE}(196)$

REF 7

A28 SIGVS

$6 L I+6 L I+A(6967)+A(6967)+A(6967)$

REF 8

A29 SIGVT

$6 \mathrm{LI}+6 \mathrm{LI} \rightarrow \mathrm{N}(8665)+11 \mathrm{C}(788)$

REF 7

A30 SIGVU

$6 \mathrm{LI}+6 \mathrm{LI} \rightarrow \mathrm{D}(2489)+10 \mathrm{~B}(498)$

REF 7

A31 SIGVV

$6 \mathrm{LI}+6 \mathrm{LI} \rightarrow \mathrm{T}(606)+9 \mathrm{~B}(202)$

REP 7 


\section{REFERENCES}

1 GEENE, USAEC REPORT UCRL -70522 (1967).

2 DUANE, USAEC REPORT BNWL-1685, 75 (1972);

YOUN ET AL. (SOV. PHYS.-JETP 12, 163 (1961).

3 ELWYN, MONAHAN, AND SERDUKE, NUCL. SCI. ENG. 63, 343 (1977).

4 GOULD, JOYCE, AND BOYCE, BULL. AM. PHYS. SOC. 20, 164 (1975); GOULD, PRIVATE COMMUNICATION (1975). See also GOULD, JOYCE, AND BOYCE, NBS SPECIAL PUBLICATION 425, p. 697 (1975); GOULD AND JOYCE, NUCL. SCI. ENG. 60, 477 (1976).

5 Z IMMERMAN, FOWLER, AND CAUGHLAN, OAP-399 (1975).

6 DAVIDSON, BERG, SIERK, AND TOMBRELLO, PRIVATE COMMUNICATION (1977).

7 L. RUBY AND T. -P. LUNG, PRIVATE COMMUNICATION (1978) .

8 E. NORBECK, NUCL. SCI. ENG. $\underline{66}, 441$ (1978).

NOTE: The <Sigma $v>$ for P6LI is up to $48 \%$ higher

in Ref. 7 than in Ref. 4, but Gould's data involves more recent data. 


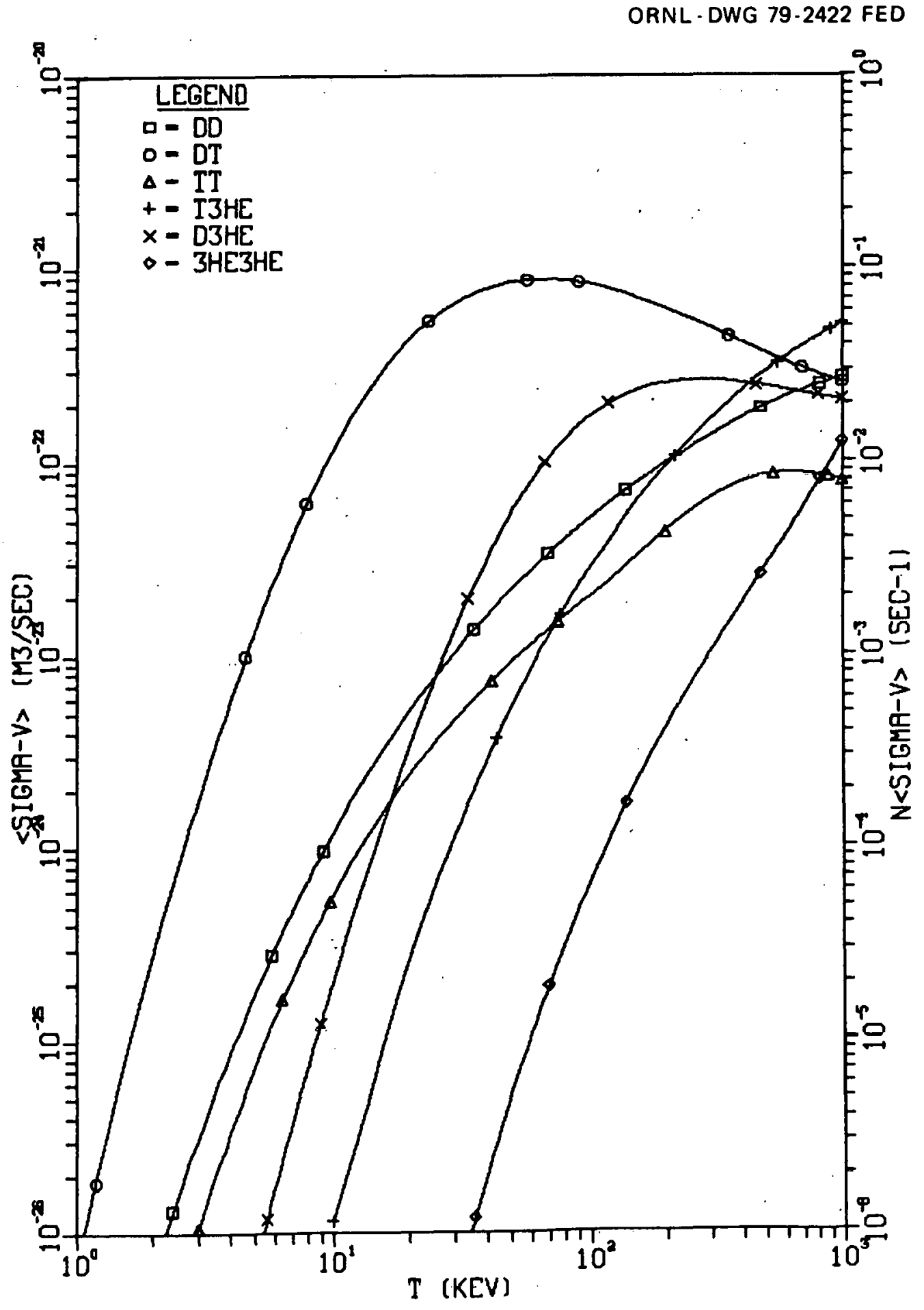

Fig. 1(a) 


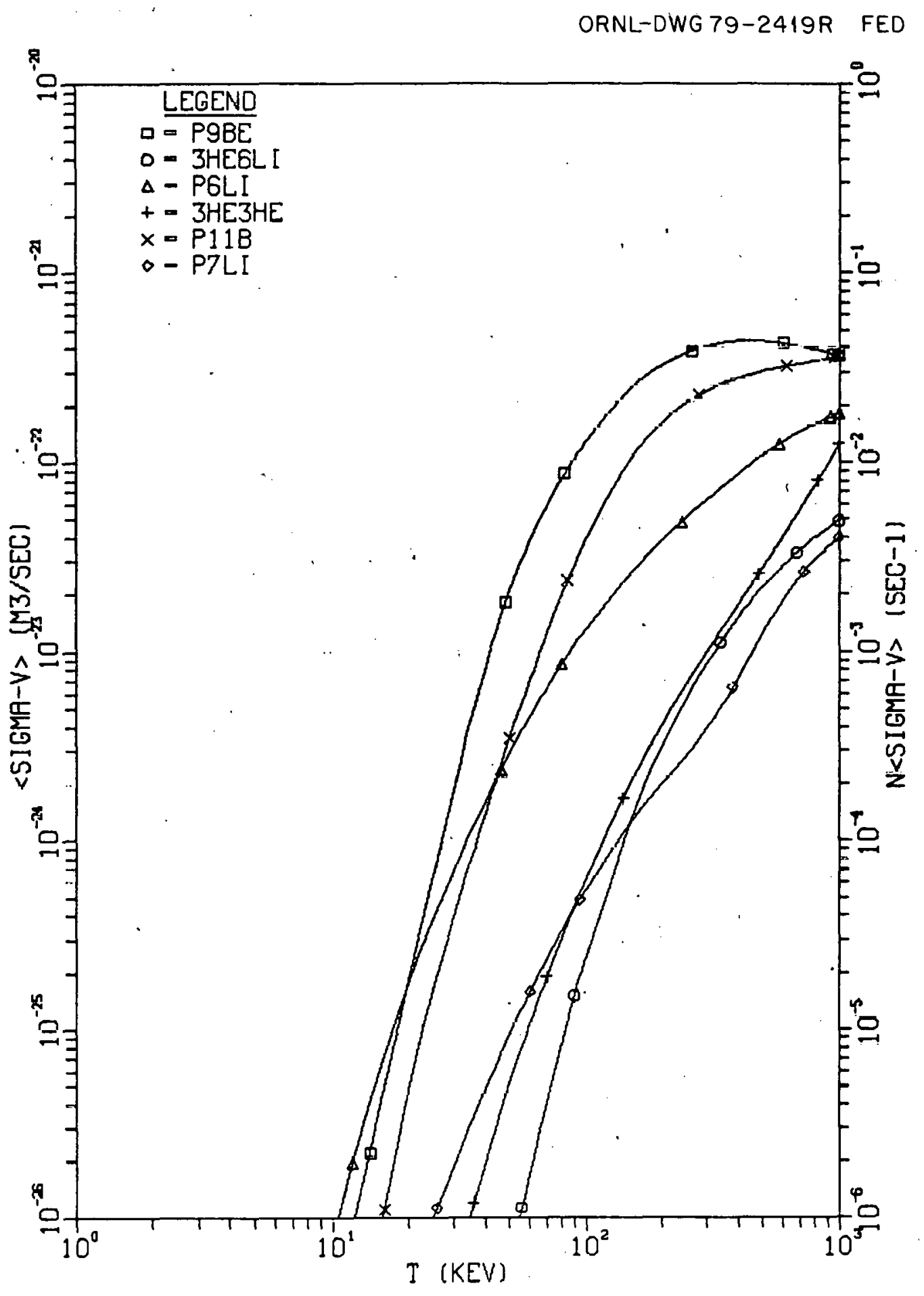

Fig. 1(b) 


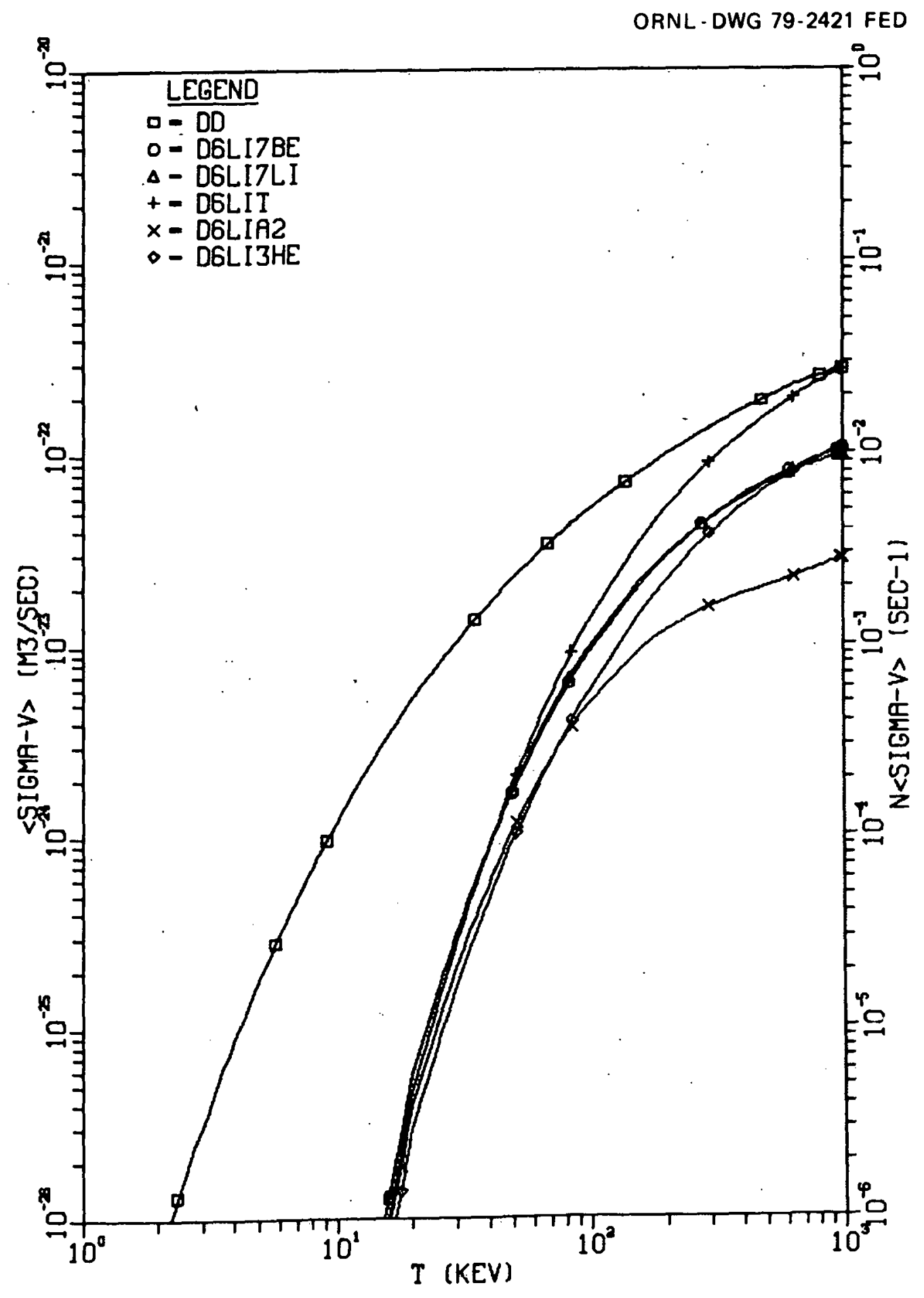

Fig. $1(c)$ 


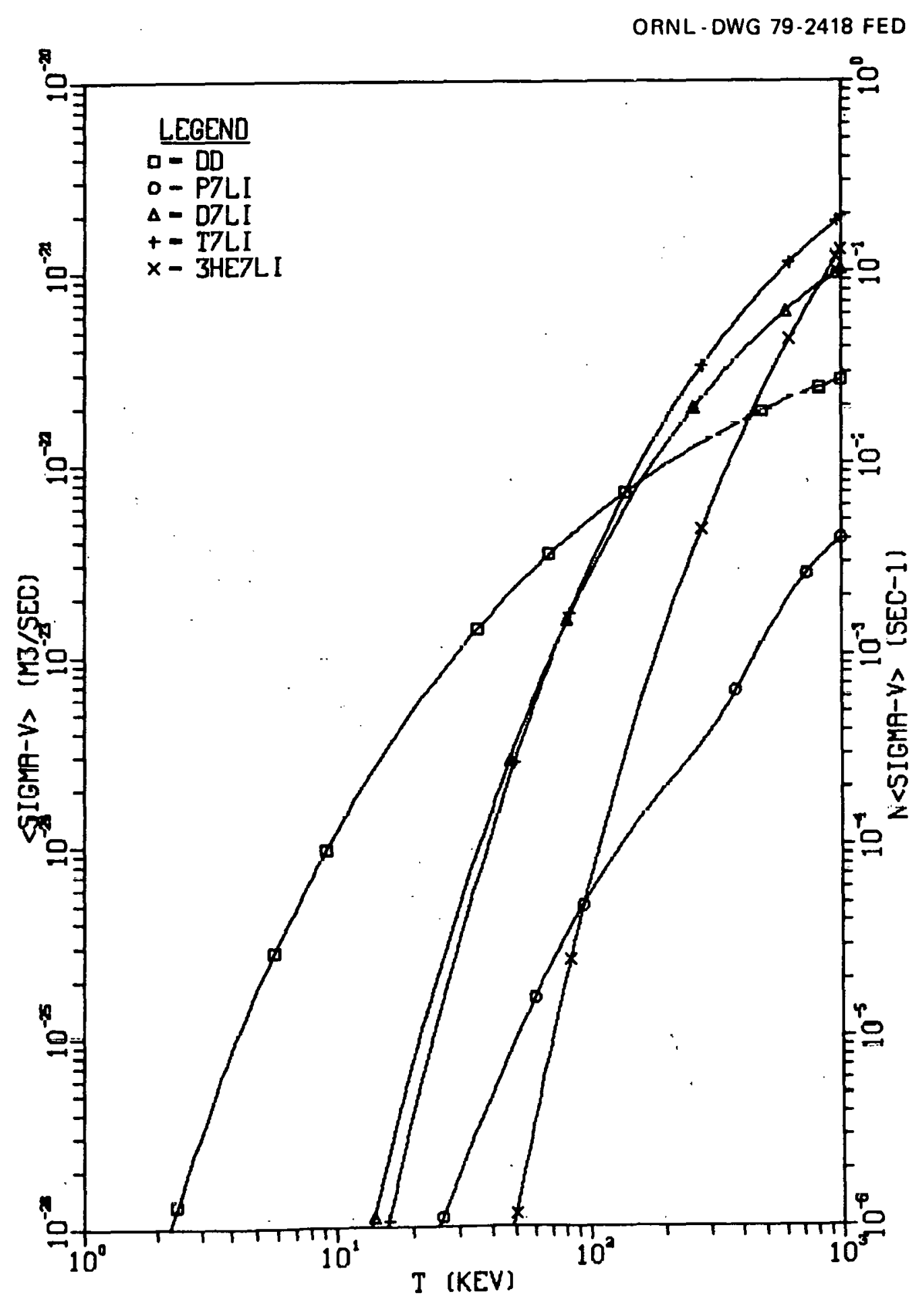

Fig. $1(d)$ 


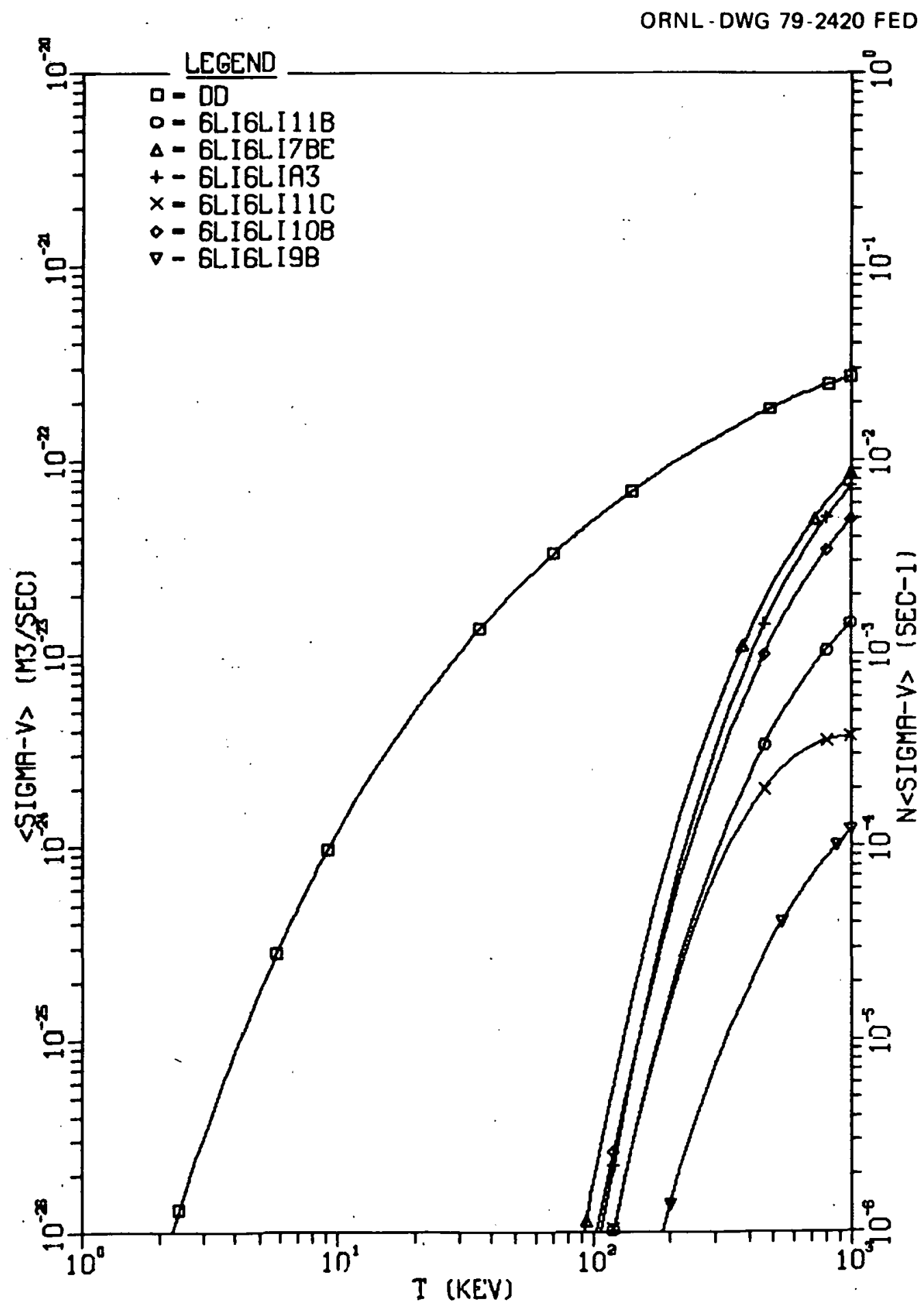

Fig. 1(e) 


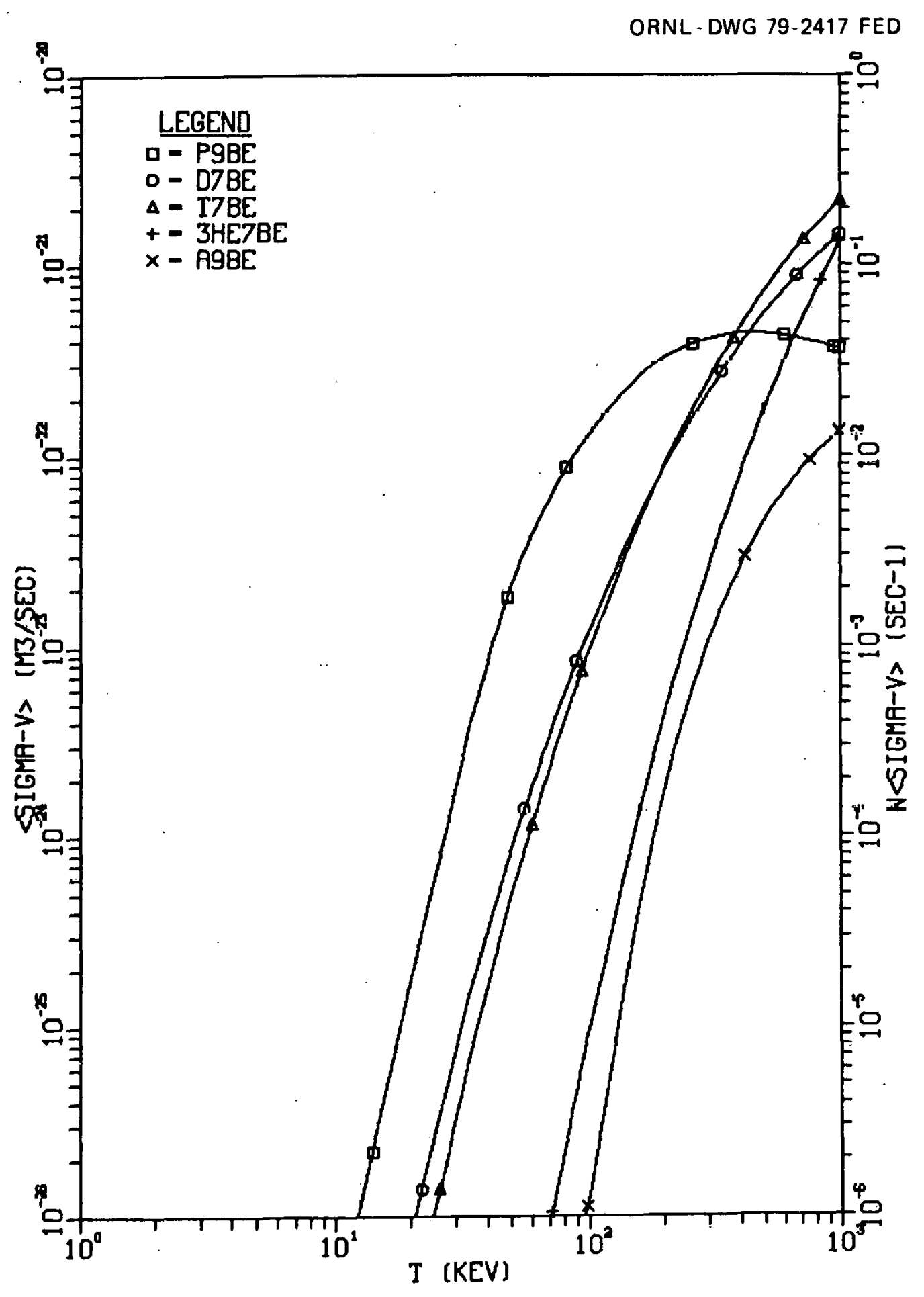

Fig. l(f) 


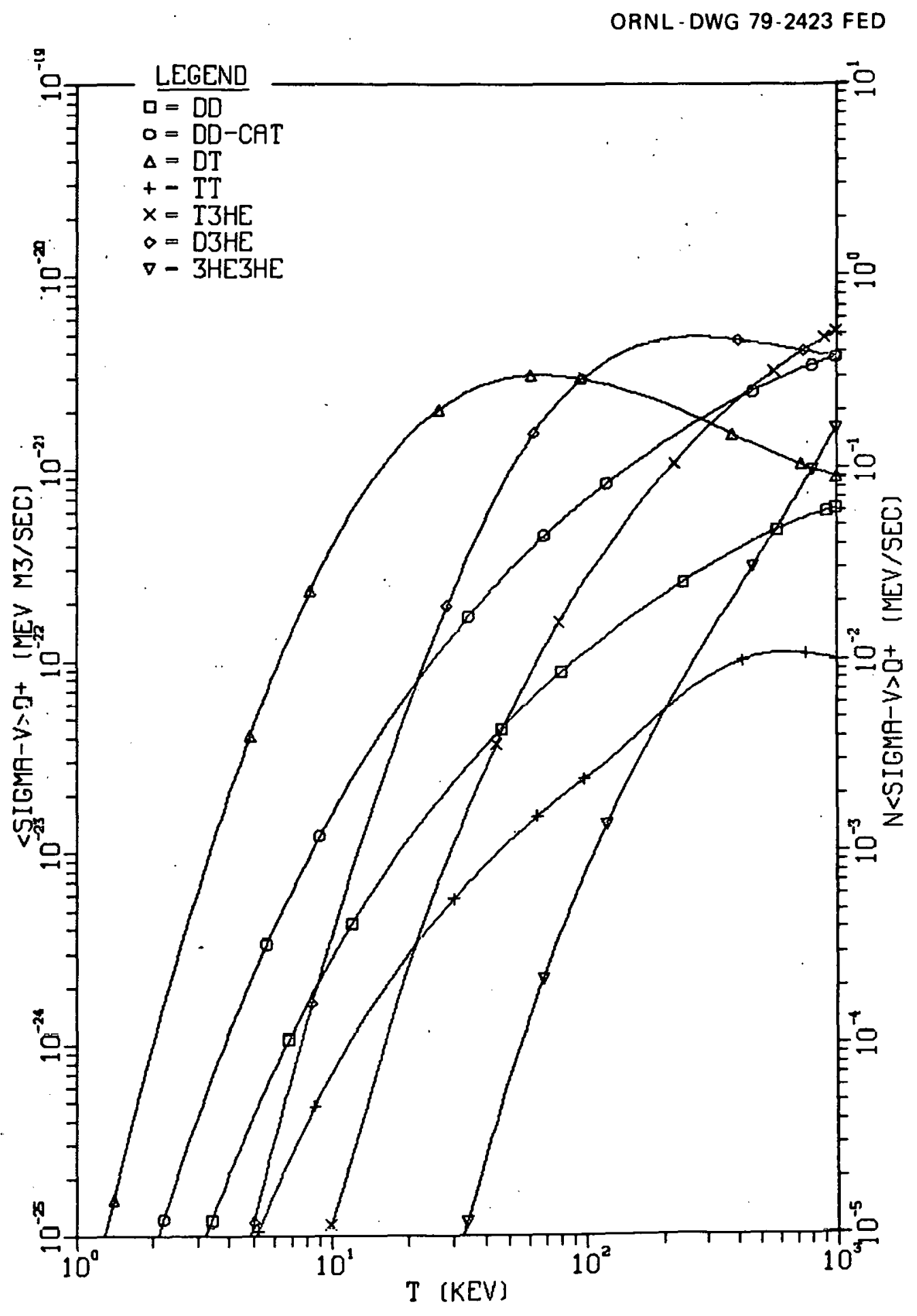

Fig. 2(a) 


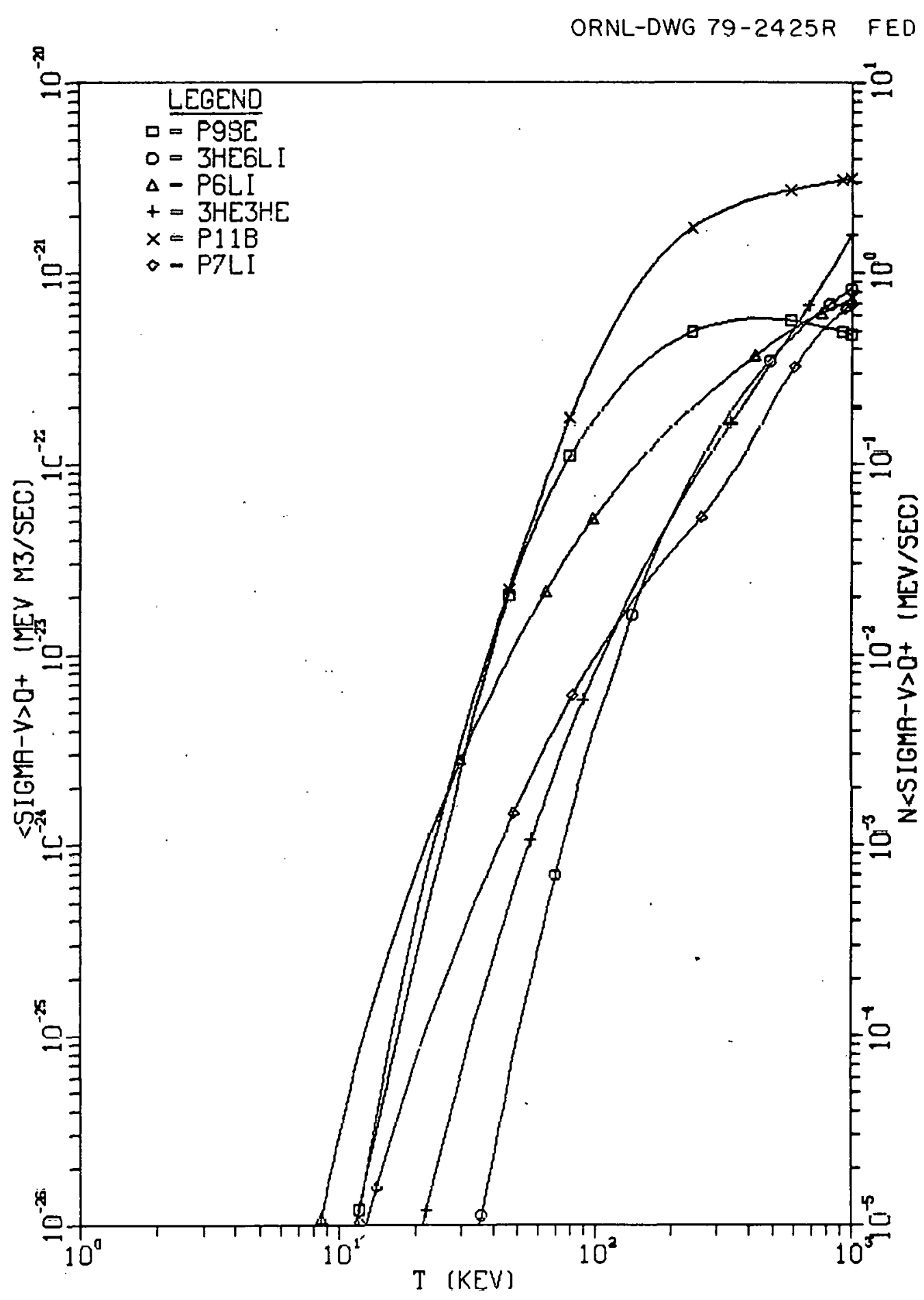

Fig. 2(b) 


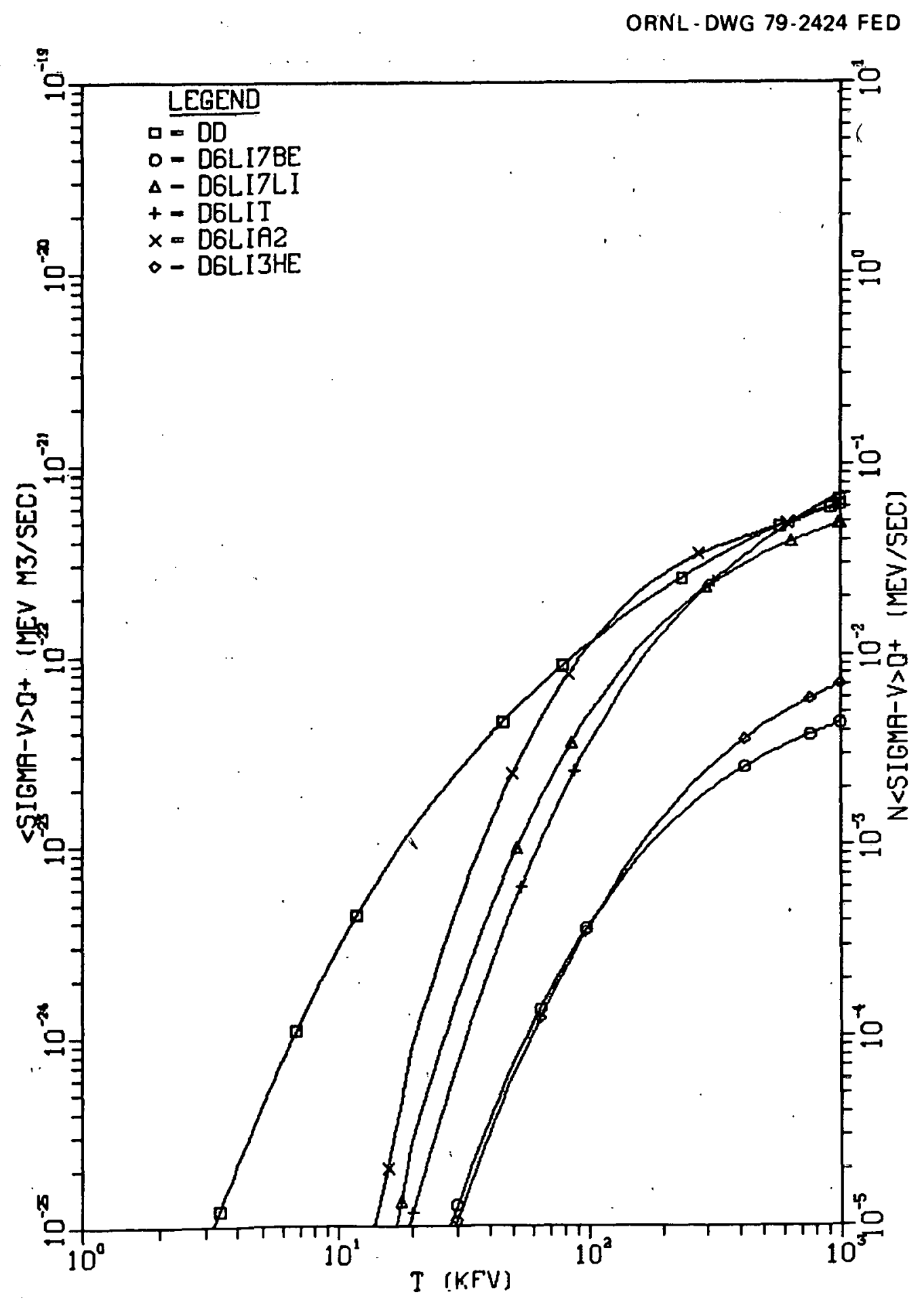

Fig. 2(c) 


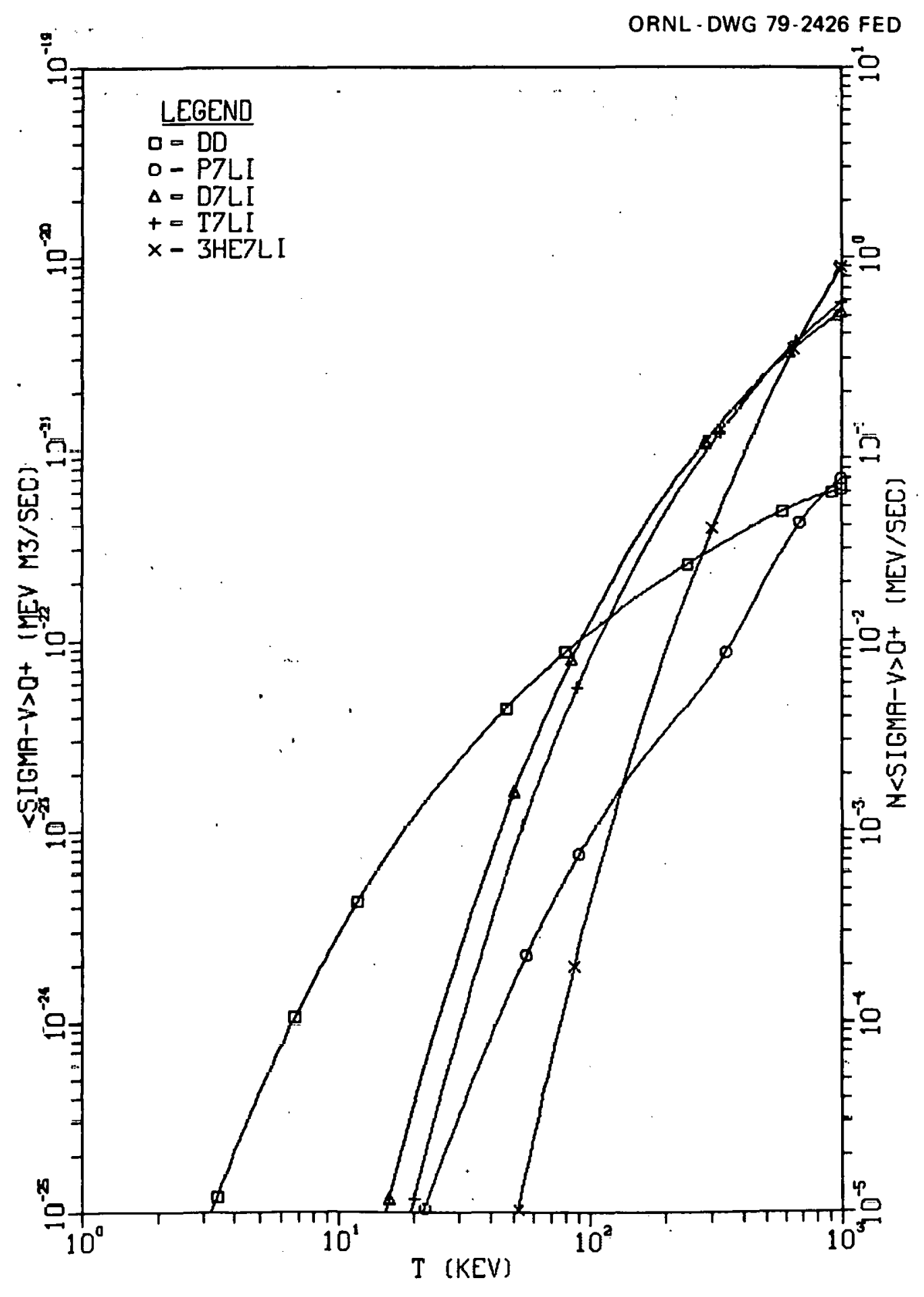

Fig. $2(d)$ 


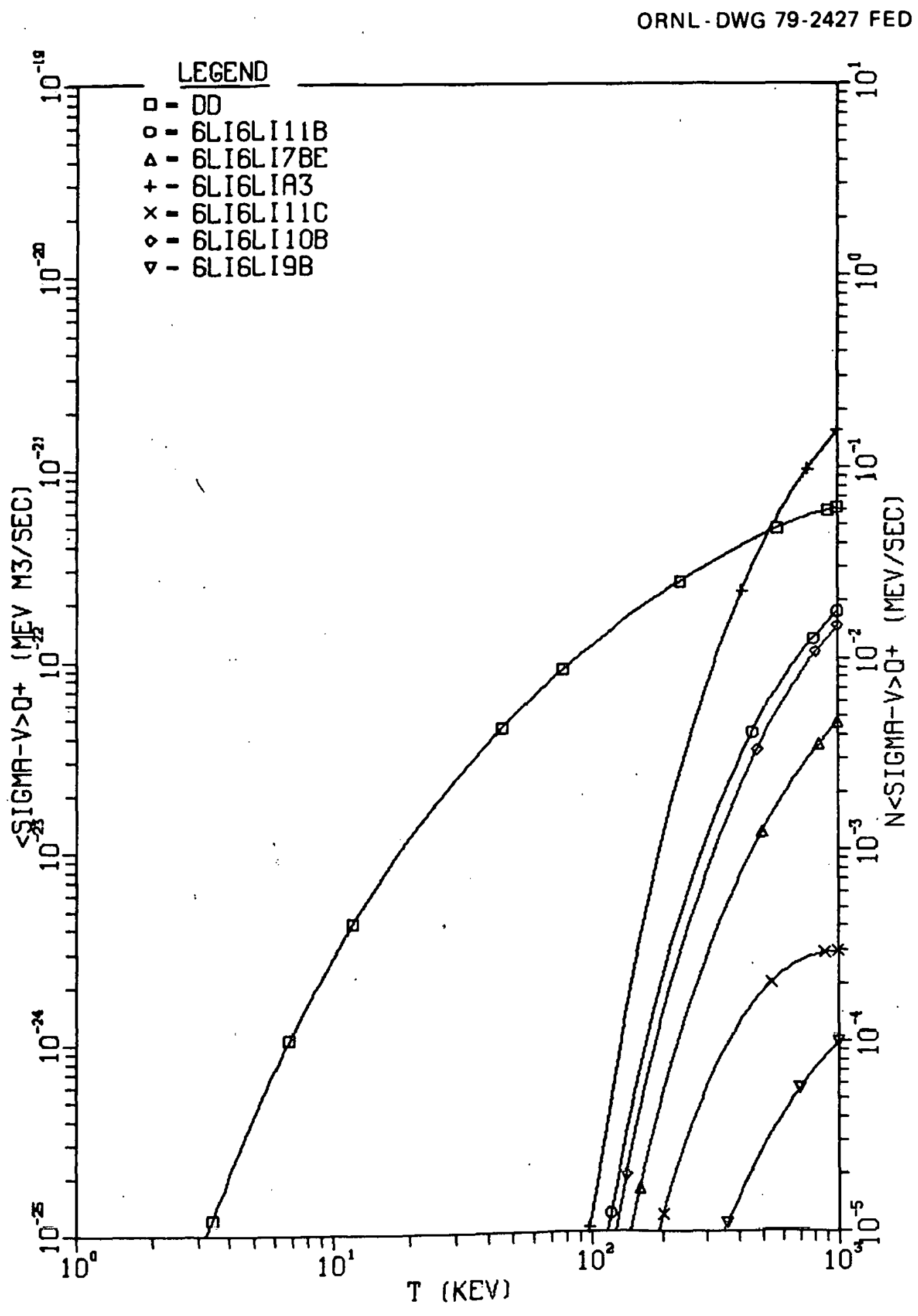

Fig. $2(\mathrm{e})$ 


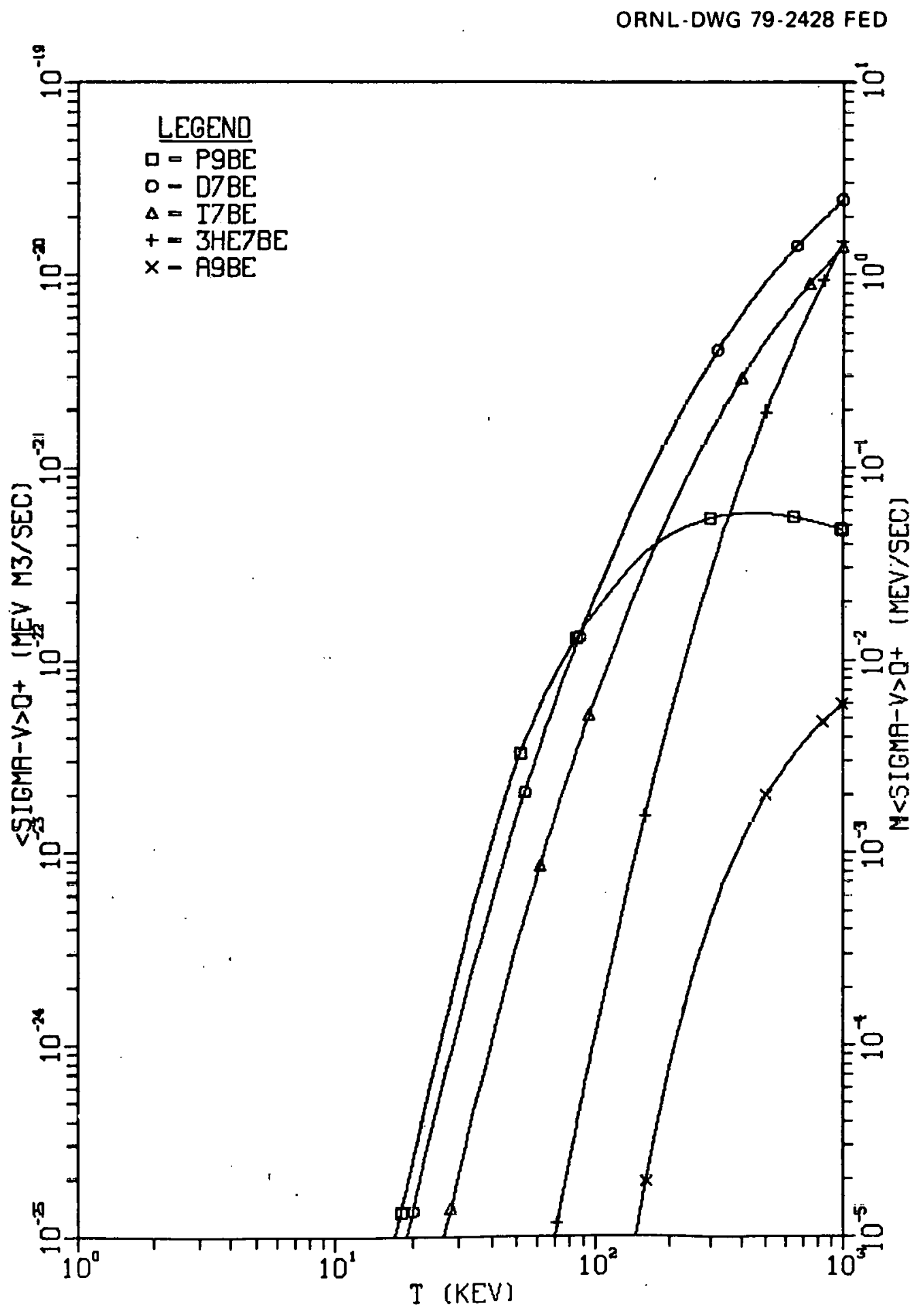

Fig. $2(f)$ 
ORNL/TM-6914

Dist. Category UC-20 a, d, g

INTERNAL DISTRIBUTION

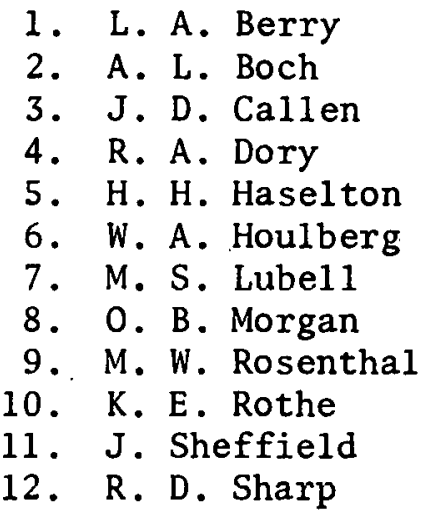

\author{
13. D. Steiner \\ 14. N. A. Uckan \\ 15-31. J. R. McNally, Jr . \\ 32-33. Laboratory Records Department \\ 34. Laboratory Records, ORNL-RC \\ 35. Document Reference Section \\ 36-37. Central Research Library \\ 38. Fusion Energy Division Library \\ Library \\ 39. Fusion Energy Division \\ Communications Center \\ 40. ORNL Patent Office
}

\section{EXTERNAL DISTRIBUTION}

41. D. J. Anthony, Energy Systems and Technology Division, General Electric Company, 1 River Road, Bldg. 23, Room 290, Schenectady, NY 12345

42. Bibliothek, Institute for Plasma Physics, 8046 Garching bei Munchen, Federal Republic of Germany

43. Bibliotheque, Service du Confinement des Plasmas, C.E.A., B.P. No. 6, 92, Fontenay-aux-Roses (Seine), France

44. Lung Cheung, Department of Electronics, University Science Center, The Chinese University of Hong Kong, Shatin, N.T., Hong Kong

45. F. Cap, University of Innsbruck, A-6020, Innsbruck, Austria

46. J. F. Clarke, Office of Fusion Energy, G-234, Department of Energy, Washington, DC 20545

47. R. W. Conn, Fusion Technology Program, Nuclear Engineering Department, University of Wisconsin, Madison, WI 53706

48. CTR Library, c/o Alan F. Haught, United Technologies Research Laboratory, East Hartford, CT 06108

49. CTR Reading Room, c/o Allan N. Kaufman, Physics Department, University of California, Berkeley, CA 94720

50. J. Narl Davidson, School of Nuclear Engineering, Georgia Institute of Technology, At lanta, GA 30332

51. Documentation S.I.G.N., Départment de la Physique du Plasma et de la Fusion Controlée, Association EURATOM-CEA sur la Fusion, Centre d'Études Nucléaires, B. P. 85 Centre du Tri, 38041 Grenoble, Cedex, France

52. W. R. Ellis, Office of Fusion Energy, G-234, Department of Energy, Washington, DC 20545

53. G. A. Emmert, Nuclear Engineering Department, University of Wisconsin, Madison, WI 53706

54. Harold K. Forsen, Exxon Nuclear Co., Inc., 777 106th Avenue, N.E., C-000777, Bellevue, WA 98009 
55. H. P. Furth, Princeton Plasma Physics Laboratory, Princeton University, Forrestal Campus, P.0. Box 451, Princeton, NJ 08540

56. Roy W. Gould, California Institute of Technology, Mail Stop 116-81, Pasadena, CA 91125

57. C. R. Head, Office of Fusion Energy, G-234, Department of Energy, Washington, DC 20545

58. Robert L. Hirsch, Exxon Research and Engineering, P.0. Box 101 , Florham Park, NJ 07932

59. Raymond A. Huse, Manager, Research and Development, Public Service Gas and Electric Company, 80 Park Place, Newark, NJ 07101

60. V. E. Ivanov, Physical-Technical Institute of the Ukranian SSR Academy of Sciences, 310108 Kharkov, U.S.S.R.

61. D. L. Jassby, Princeton Plasma Physics Laboratory, P.0. Box 451, Princeton, NJ 08540

62. A. Kadish, Office of Fusion Energy, G-234, Department of Energy, Washington, nC, 20.545

63. E. T. Karlson, Department of Technology, Box 534, S-75121 Uppsala, Sweden

64. Y. Kiwamoto, Research Institute for Energy Materials, Yokohama National University, Yokohama 232, Japan

65. R. N. Kostoff, Room 509, Office of Energy Research, Department of Energy, 401 First St., N.W., Washington, DC 20545

66. L. M. Kovrizhnykh, Lebedev Institute of Physics, Academy of Sciences of the U.S.S.R., Leninsky Prospect 53, Moscow, U.S.S.R.

67. Guy Laval, Groupe de Physique Theorique, Ecole Polytechnique, 91 Palaiseau, Paris, France

68. Library, Centre de Recherches en Physique des Plasma, 21 Avenue des Bains, 1007, Lausanne, Switzerland

69. Library, Culham Laboratory, United Kingidom Aromlc Energy Aulhurily, Abingdon, Oxur, ox14 3DB, United Kingdom

70. Library, FOM-Institut voor Plasma-Fysica, Rijnhuizen, Jutphaas, Netherlands

71. Library, Institute for Plasma Physics, Nagoya University, Nagoya, Japan 464

72. Library, International Centre for Theoretical Physics; Trieste, Italy

73. Library, Laboratorio Gas Tonizzati, Frascati, Itàly

74. J. G. Lominadzc, Acadcmy of Sciences of the Georgian S.S.R., 8 Dzerzhinski St., 38004, Tbilisi, U.S.S.R.

75. O. P. Manley, Office of Fusion Energy, G-234, Department of Energy, Washington, DC 20545

76. D. G. McAlees, Exxon Nuclear Co., Inc., 777 106th Avenue, N.E., Bellevue, WA 98009

77. J. E. McCune, School of Engineering, Department of Aeronautics and Astronautics, B1dg. 37-391, Massachusetts Institute of Technology, Cambridge, MA 02139

78. A. T. Mense, Subcommittee on Energy Research and Production, B-374, Rayburn House Office Building, Washington, DC 20515

79. C. Mercier, Service du Theorie des Plasmas, Centre d'Études Nucléaires, Fontenay-aux-Roses (Seine), France

80. M. Murphy, Office of Fusion Energy, G-234, Department of Energy, Washington, DC 20545 
81. D. B. Nelson, Office of Fusion Energy, G-234, Department of Energy, Washington, DC 20545

82. K. Nishikawa, Institute for Fusion Theory, Hiroshima University, Higashisendamachi, Hiroshima, Japan

83. B. Outten, Jr., Western Metal Products Company, 1300 Weber Street, Orlando, FL 32803

84. R. E. Papsco, Grumman Aerospace Corp., 101 College Road, Princeton, NJ 08540

85. 0. Petrus, Supervisor, C.T.R., Theory Group, Plasma Physics Laboratory, "Alexandru Ion Cuza," University, Iasi, Romania, 6600

86. D. Pfirsch, Institute for Plasma Physics, 8046 Garching bei Munchen, Federal Republic of Germany

87. Plasma Physics Library, c/o Dr. Philip Rosenau, Department of Mechanical Engineering, Technion-Israel, Institute of Technology, Haifa, Israel

88. Plasma. Physics Group, Department of Engineering Physics, Australian National University, P.O. Box 4, Canberra A.C.T. 2600, Australia

89. A. Rogister, Institute for Plasma Physics, KFA, Postfach 1913, D-5170, Julich 1, Federal Republic of Germany

90. W. Sadowski, Office of Fusion Energy, G-234, Department of Energy, Washington, DC 20545

91. V. D. Shafranov, I. V. Kurchatov Institute of Atomic Energy, 46, Ulitsa Kurchatova, P.0. Box 3402, Moscow, U.S.S.R.

92. Yu. S. Sigov, Institute of Applied Mathematics of the U.S.S.R. Academy of Sciences, Miuskaya, Sq. 4, Moscow A-47, U.S.S.R.

93. W. M. Stacey, Jr., School of Nuclear Engineering, Georgia Institute of Technology, Atlanta, GA 30332

94. L. D. Stewart, Princeton Plasma Physics Laboratory, P.0. Box 451, Princeton, NJ 08540

95. J. B. Taylor, Culham Laboratory, United Kingdom Atomic Energy Authority, Abingdon, Oxon, Ox14 3DB, United Kingdom

96. Thermonuclear Library, Japan Atomic Energy Research Institute, Tokai, Naka, Ibaraki, Japan

97. K. Uo, Plasma Physics Laboratory, Kyoto University, Gokasho, Uji, Kyoto, Japan

98. Francisco Verdaguer, Director, Division of Fusion, Junta de Energia Nuclear, Madrid 3, Spain

99. K. M. Zwilsky, Office of Fusion Energy, G-234, Department of Energy, Washington, DC 20545

100. Office of Assistant Manager, Energy Research and Development, Department of Energy, Oak Ridge Operations Office, Oak Ridge, TN 37830

101-345. Given distribution as shown in TID-4500, Magnetic Fusion Energy (Distribution Category UC-20 a, d, g: Plasma Systems, Fusion Systems, and Theoretical Plasma Physics) 